\title{
Quelques aspects des études hydrauliques des aménagements du Bas-Rhône
}

\section{Some aspects of the hydraulic studies carried out in connection with the development of the Lower Rhône}

\author{
par Cx. GEMAEHLING, \\ INGÉNIEUR DES PONTS ET CHAUSSÉES \\ DERCTEUR-ADYOINT DE LA COMPAGNIF NATIONATE DU RHONE
}

\begin{abstract}
L'aménagement continu sur près de $100 \mathrm{~km}$ de long, d'un grand flewve au débit solide abondant, a nécessité des études hydrauliques très varices, dont certaines permettent d'ailleurs une utile confrontation entre les études préalables théoriques ou expérimentales (sur modèles réduits) et les observations faites après achèbement des travaux.

L'auteur relate certaines études à portée générale concernant les ouvrages des aménagements mixtes récents din Bas-Rhône (notamment BaixLe Logis Neuf, Beauchastel et Pierre-Bénite), et procède à certaines comparaisons avec les aménagements phus anciens (Donzère-Mondragon et Montélimar).

Ces études portent principalement sur les aspects suivants :

- Le barrage et son mode de construction dans le lit du Rhône, en liaison avec les problèmes posés par le passage de la ravigation, l'éconlement des crues et le cheminement du débit solide;

- les conditions d'établissement et d'exploitation des prises d'eau du canal de dérivation, de manière da éviter notamment l'engravement de la retenue et du seuil

- enfin, les problemes spécifiques liés an groupement de l'usine, du déchargeur et de l'ćcluse en un bloe unique barrant le canal de dérination: suppression des courants traversiers pouvant gêner les bateaux à l'approche des usines, bonne alimentation du sas des échuses, utilisation des déchargeurs pour réduire les ondes provoquées par les changements de régime des centrales hydroélectriques.

Les études dont il est rendu compte montrent notamment l'importance que revétent les problèmes posés par le passage de la navigation et le cheminement du débit solide dans it conception, la réalisation et l'exploitation des aménagements hydroélectriques du bas-Rhóne.
\end{abstract}

The development of a continutus atretch of nearly $100 \mathrm{~km}$ along this big, heavily sedimentladen river has involved a wide range of different hydraulic studies, some of which have provided a valuable basis for a comparison between previous theoretical or experimental scale model research and observations recarded since the completion of the work on the site. The Author describes a number of general investigations connected with the structures in recent multi-purpose development projects on the Lower Rhone (in particular Baix-Le Logis Yeuf, Beanchastel and Pierre-Bénite), and compares some of their aspects with older projects, such as Donzère-Mondragon and Montélimar. These investigations were mainly concerned with the following points:

(i) The dam and the method used to build it on the bed of the river Rhone, with due regard to problems arising from navigation requirements, the discharging of flood flows, and the conveying of the river's sediment load.

(ii) Conditions governing the provision and running of the diversion canal water intakes, especially as regards silt prevention in the im. pounded water and on the sill.

(iii) Problems specifically associated with the grouping of the power house, relief works and lock as a single unit extending right across the diversion canal, such as the prevention of any cross-currents liable to interfere with shipping in the power house approaches, efficient water supply systems for the lock chambers, and the use of relief works to reduce surge waves due to variations in power station operation.

The investigations described in this articte show the importance of problems associated with river shipping, the conveying of sediment through the installation, and the construction and running of the hydro-power schemes along the Lower Rhone. 
L'équipement du bas Rhône, entrepris il y a quinze ans, a porté jusqu'à présent sur le troncon d'une centaine de kilomètres de long compris entre le confluent de l'Isère et Mondragon (tiers central du bas Rhône). Rappelons qu'il s'agit des chutes suivantes que nous citons dans l'ordre des dates de mises en service, c'est-à-dire d'aval en amont: Donzère-Mondragon (1952), Montélimar (1957), Baix-Le Logis Neuf (1960), ainsi que celle en construction de Beauchastel (mise en eau prérue : 1963). Le programme des travaux se poursuit sur le tiers amont avec la chute de Pierre-Bénite (mise en eau prévue: 1965 ) alors que les études des autres chutes des tiers amont et aval sont dejà largement amorcées.

Les schémas d'aménagement qui ont été adoplés pour chacune de ces chutes présentent sans doute beaucoup d'analogie. Ils comportent en effet les ouvrages principaux suivants (fig. 1) :

- un barrage de retenue, qui règle le débit laissé dans le troncon aval du fleuve courtcircuité par la dérivation et relève les eaux à l'entrée de la prise;

- en amont de celle-ci, une retenue longue de 5 a $10 \mathrm{~km}$ avec les endiguements, les ouvrages, et, le cas échéant, les dragages et dérasements de berges nécessaires pour contenir les eaux du fleuve et assurer l'écoulement des affluents;

- un canal de dérivation issu de la prise d'eau, et qui conduit, sur un parcours de plusieurs kilomètres, le débit dérivé jusqu'au point choisi pour la restitution dans le fleuve;

-. les ouvrages d'utilisation et de franchissement de la chute: usine hydroélectrique équipée de groupes Kaplan, écluse et ouvrages annexes (notamment déchargeur), groupés en un bloc unique qui partage la dérivation en un canal d'amenée et un canal de fuite.

L'importance et la diversité des ouvrages concus à la taille du fleuve, la nécessité d'assurer une sécurité absolue aux riverains et une excellente régularité à l'exploitation industrielle, enfin la situation propre à chaque chute nous ont amenés à entreprendre des études hydrauliques de toules sortes: essais sur modèles réduits (1), études et calculs théoriques, recherches opérationnelles effectuées sur calculatrice électronique, observations faites sur place.

Il nous a paru utile de rendre compte de certaines de ces études, en essayant de dégager les idées générales qui résultent de la confrontation des résultats obtenus pour diverses chutes et en insistant sur les problèmes qui sont spécifiques aux aménagements mixtes du Rhône.

Nous ne tenterons pas de faire un inventaire, mème sommaire, des principales études faites, car il risquerait d'être à la fois superficiel et fastidieux dans sa longueur. En particulier, les études hydrauliques relatives à la conception des ouvrages principaux et à leur mise au point (notamment l'usine hydroélectrique) seront à peu près complètement passées sous silence, ainsi que tout ce qui a trait à l'hydraulique souterraine.

Nous nous bornerons donc à étudier successivement les aspects suivants :

- le barrage et son mode de construction dans le lit du Rhône, ce qui nous permettra d'évoquer les problèmes posés par le passage de la navigation, l'écoulement des crues et le cheminement du débit solide;

- la prise d'eau du canal de dérivation et les conditions de son exploitation de manière à éviter notamment l'engravement de la retenue et du seuil;

-.- enfin, les problèmes spécifiques liés au groupement de l'usine, du déchargeur et de l'écluse en un bloc unique barrant le canal de dérivation : suppression des courants traversiers pouvant gêner les bateaux à l'approche des usines, bonne alimentation du sas des écluses, utilisation des déchargeurs pour réduire les ondes provoquées par les changements de régime des centrales hydroélectriques.

En définitive, les études dont nous rendons compte traduisent l'importance que revêtent les problèmes posés par le passage de la navigation, l'écoulement des crues et le cheminement du débit solide, dans la conception, la réalisation et l'exploitation des aménagements hydroélectriques du Bas-Rhône.

(1) Ces essais ont en général été confiés : au Laboratoire National d'Hydraulique de Chatou pour les prises et les barrages, et les problèmes liés au eheminement du débit solide; au Laboratoire de la Sogrear à Grenoble pour les usines et les écluses, la restitution des dérivations et les problèmes d'ondes dans les canaux, De son côté, la Compagnie dispose d'un petit laboratoire à Gerland (Rhône) où elle étudie directement certains ouvrages : notamment les déchargeurs, les endiguements submersibles bordant les retenues ainsi que le réaménagement du débouché de certains affluents (Lez, Eyrieux, Roubion, Isere). 


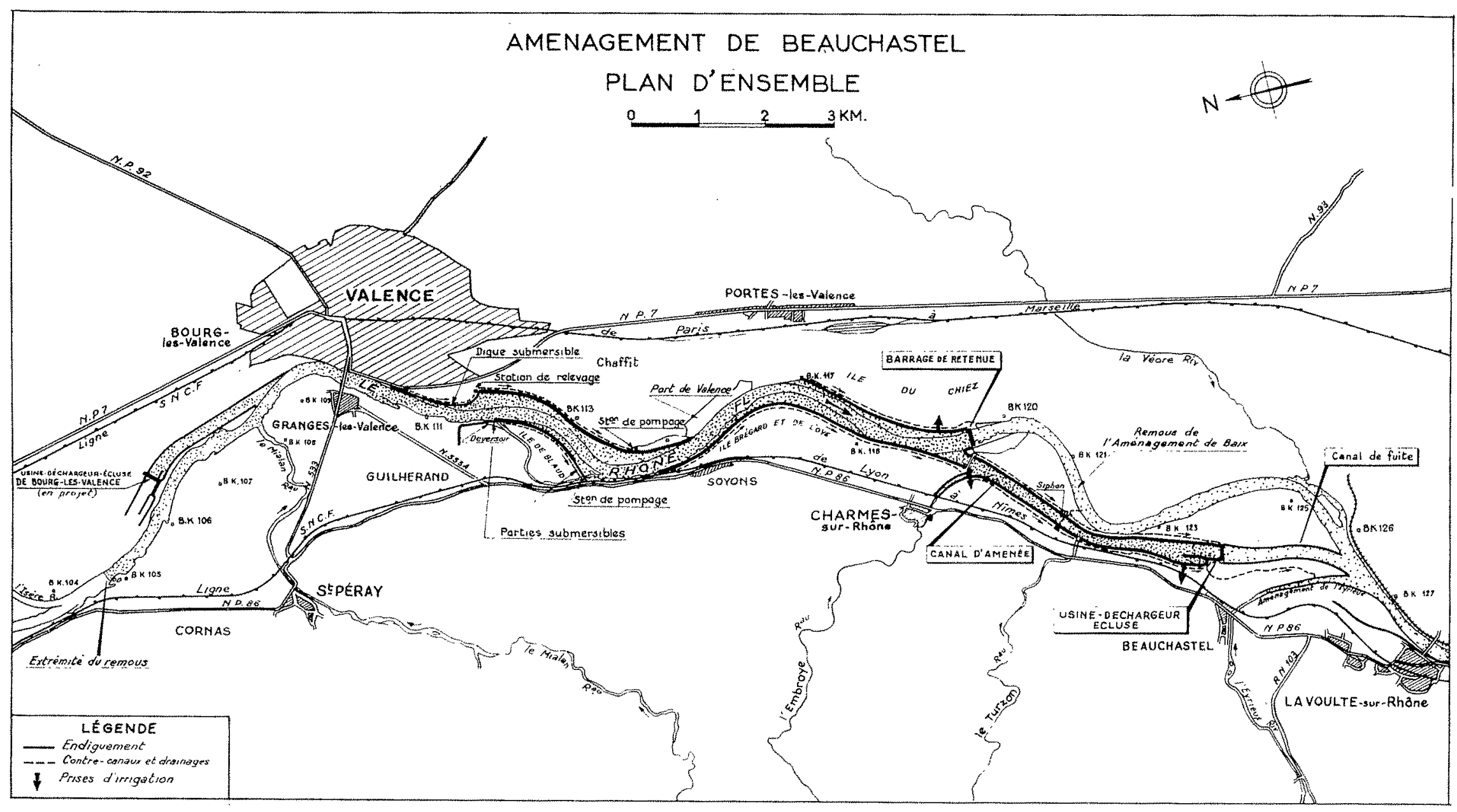

FIG. 1 


\section{1. - BARRAGE ET RETENUE}

\subsection{Les barrages de retenue.}

Les barrages dont nous avons rappelé le rôle en quelques mots sont, en général, établis en travers du lit mineur du flenve et sont tous équipés de bouchures mobiles : fermées, les vannes permettent de relever le niveau des eaux en période d'étiage ou d'eaux moyennes; partiellement. ou totalement ouvertes, elles laissent passer les crues qui continuent d'emprunter l'ancien cours du fleuve. L'infrastructure se compose done essentiellement de piles massives en rivière, qui supportent les vannes, et d'un radier établi an niveau moyen du lit.
Nous avons réuni dans le tableau I les principales caractéristiques chiffrées relatives aux barrages du Bas-Rhône. En. dehors de celui de Donzère, aux passes inégales équipées de vannes segments, tous les onvrages postérieurs comportent des passes d'égale largeur, équipées de vannes wagons à deux corps (dites vannes à crochet). Celui de Beauchastel (fig. 2), en cours de construction, caractérise bien la conception adoptée.

Les études hydrauliques nécessitées par la mise au point d'un projet de barrage sont très diverses. En dehors de ce qui sera dit plus loin au sujet du processus de construction, nous nous

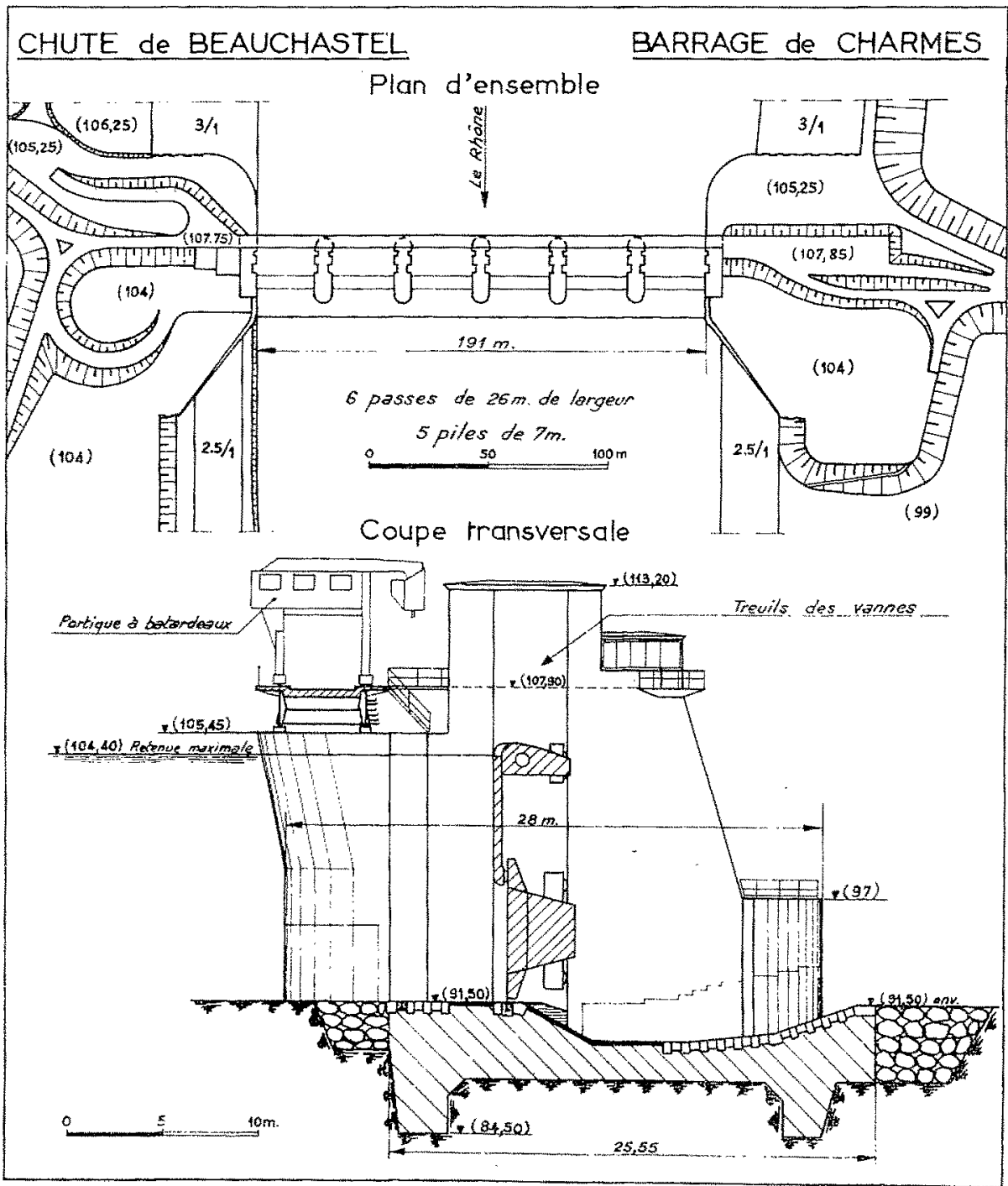

FIC. 2

UN EXEMPLE

DE BARRAGE DE RETENUE (barrage de Charmes).

Plan schématique de l'ouvrage at coupe transversale d'une passe. 
Tabiene: I. - Barrages du Bas-Rhòne.

Ce tableau rassemble les caractérititiques essentielles (géométriques el hydrologiques) des barrages de retenue du Bas-Rhône. L'appellation habituelle de chaque barrage est indiquée, entre parenthèses, au-dessous du nom de l'aménagement arquel il appartient.

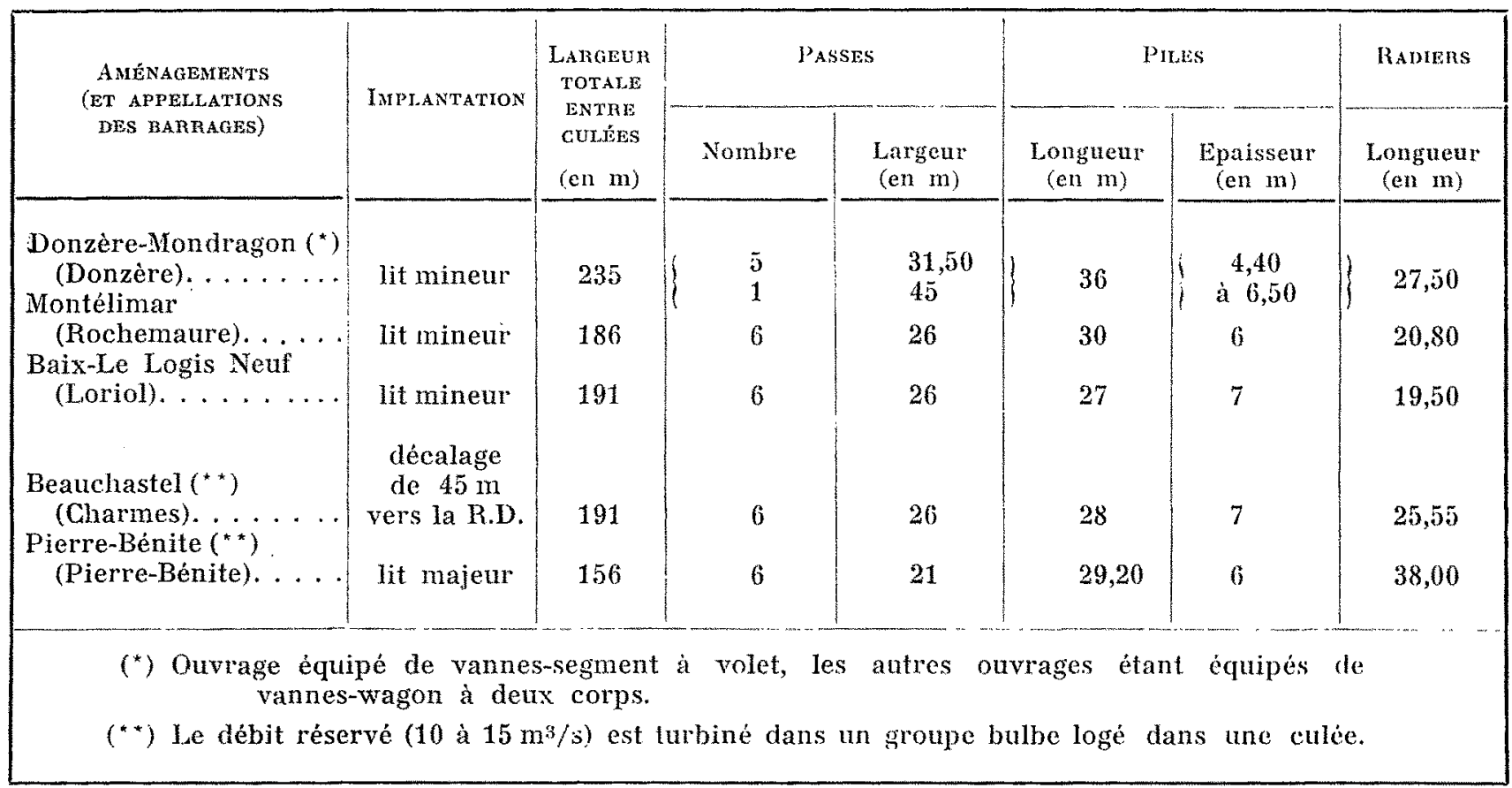

\begin{tabular}{|c|c|c|c|c|c|c|}
\hline \multirow{2}{*}{ AMÉNAGEMENTS } & \multicolumn{2}{|c|}{$\begin{array}{c}\text { Havtevo } \\
\text { DE la RETENuE Nonmale } \\
\text { (au-dessus du scuil) }\end{array}$} & \multicolumn{2}{|c|}{ Crug maxmate } & \multicolumn{2}{|c|}{ Processus DE construction } \\
\hline & $\begin{array}{l}\text { Minimale } \\
\text { (en m) }\end{array}$ & $\begin{array}{l}\text { Maximale } \\
\text { (csi } \mathrm{m})\end{array}$ & $\begin{array}{c}\text { Débit } \\
\text { (en } m * / s)\end{array}$ & $\begin{array}{l}\text { Hauteur } \\
\text { amont: } \\
\text { au-dessus } \\
\text { du sewil } \\
\text { (en m) }\end{array}$ & $\begin{array}{l}\text { Nombre } \\
\text { de phases } \\
\text { principales }\end{array}$ & Dérivation provisoire \\
\hline Donzère-Mondragon. & 8,50 & 8,50 & 10000 & 12,50 & 5 & non \\
\hline Montélimar. . . . & 12,00 & 12,00 & 10000 & 11,60 & 4 & oui (en rive Iroite) \\
\hline Baix-Le Logis Neuf & 11,00 & 12,75 & 10000 & 11,30 & 4 & oui (en rive droite) \\
\hline Beauchastel... & 11,50 & 12,90 & 9500 & 10,10 & 3 & $\begin{array}{l}\text { oui (élargissement du } \\
\text { lit en rive gauche) }\end{array}$ \\
\hline Pierre-Bénite. . . . . & 10,75 & 10,75 & 7500 & 12,35 & 1 & oui (en rive droite) \\
\hline
\end{tabular}

contenterons d'évoquer brièvement certains problèmes posés par les piles et les radiers, et l'écoulement des grandes crues à travers l'ouvrage, en passant sous silence notamment tout ce qui concerne l'étude détaillée de l'écoulement au voisinage des vannes.

1.1.1. Les prLes ont une forme simple : abstrac- 


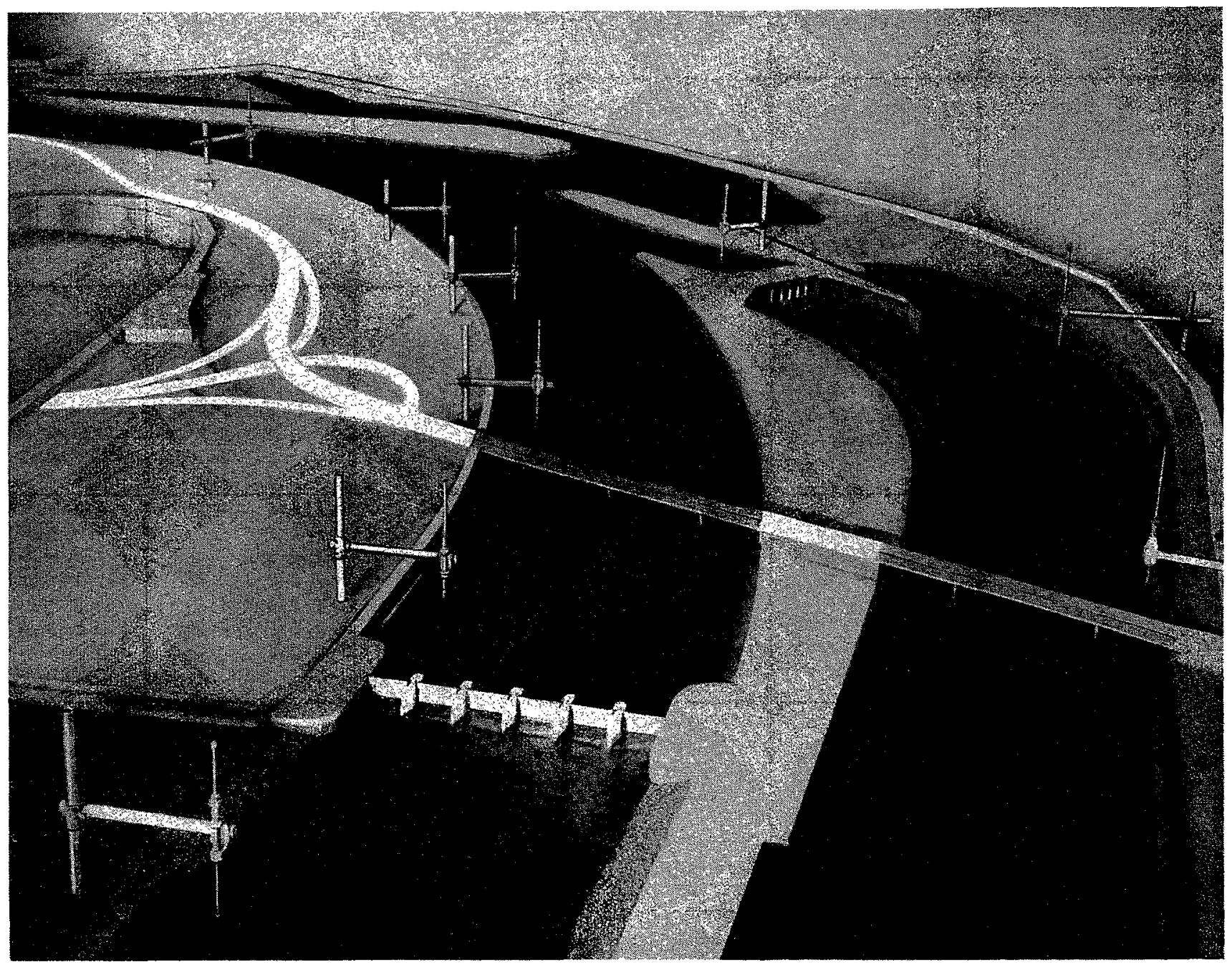

(Photo Laboratoire national d'Hydraulique.)

Fig. 3

UN EXEMPLE DE MODF́LE RÉDUTT :

modèle à l'échelle $1 / 100$ des ouvrages principaux de l'aménagement de Pierre-Bénite.

Vue partielle du modèle représentant les ouvrages après achèvement de leur construction. En fait, le modèle s'étend : ver's l'amont, jusqu'au-delà du confluent de la Saône; vers l'aval, sur une longueur suffisante pour que l'écoulement dans l'évacuateur de crues of dans le canal de fuite soit représenté correctement. Au premier plan: le barrage de retenue, établi sur une déviation du fleuve; dans le quart supérieur droit : l'usine (avec son court canal d'amenée), l'écluse et les garages de navigation; d l'arrière-plan: le chenal d'accès et les darses du port Edouard-Herriot; dans la partie médiane de la photo: l'autoroute de sortie sud de Lyon, avec ses ponts.

Lion faite des rainures de vannes et batardeaux, ce sont des parallélipipèdes rectangles complétés par des avant-becs et des arrière-becs demicylindriques. Les essais ont montré que des formes plus compliquées n'apportaient pas de réduction appréciable des pertes de charge à la traversée des barrages.

Cependant, au barrage de Pierre-Bénite, les essais sur modèle réduit ont conduit à munir les piles d'un arrière-bec effilé (fig. 3) pour éviter, dans certains cas de fonctionnement, l'appari- tion systématique d'oscillations transversales du plan d'eau (2) (seiches multinodales), qui paraissent induites par des tourbillons alternés prenant naissance en aval des piles (fig. 4). Pour les autres barrages, les conditions de débit et de

(2) S'il ne semble pas avoir été étudié complètement, ce phénomène a déjà été signalé par plusieurs auteurs [5].

Les chiffes qui figurent entre crochets [] dans la présente communication renvoient tous à la bibliographie sommaire citée in fine. 
hauteur d'eau susceptibles de provoquer ces phénomènes ne se trouvent pas réalisées (3).

1.1.2. LES RADIERS doivent avoir une forme telle que l'écoulement de l'eau à travers les barrages ne risque pas de provoquer à leur voisinage des affouillements dangereux. Les risques sont évidemment beaucoup plus grands pour les ouvrages fondés sur une couche de gravier ou de mollasse très affouillable (Pierre-Bénite) que pour les ouvrages du tiers central fondés sur une roche résistante.

Divers cas de fonctionnement doivent être étudiés avec soin :

(3) Il semble toutefois qu'un tel éconlement se soit manifesté une seule fois à Donzère depuis la mise en service du barrage pour un cas d'exploitation (niveau et débit) tout à fait exceptionnel. Il a suff de fermer partiellement une des six passes ouvertes pour que ce phénomène spectaculaire, mais sans gravité, cesse immédiatement.
-- en retenue normale, les vannes sont partiellement ouvertes pour les débits du fleuve supérieurs au débit turbinable; le plan d'eau aval étant beaucoup plus bas que le plan d'eau amont, l'écoulement est très rapide (15/ms sous le couteau); mais l'énergie se dissipe sur une assez courte distance :

en grandes crues, les vannes sont completement ouvertes; l'écoulement à la traversée du barrage est moins rapide, mais l'accroissement de la puissance d'érosion règne sur une plus grande longueur. L'indisponibiliti d'une passe (pour entretien ou réparation) peut provoquer des écoulements dissymétriques favorisant les érosions;

-.- I'utilisation partielle de l'ouvrage lors de la mise en eau de la chute donne lieu pendani quelques mois à des écoulements très défavorables pouvant engendrer d'importants affouillements : les crues, qui sont concen-
Fig. 4

PHẾNOMÈNE D'OSCILLATION DUY PLAN D'EAU

au barrage de Piemre-Bénite (étude sur modèle réduit).

Vue partielle de l'écoulement à travers le barrage complètement ouvert. Les conditions de débit et de niveau aval sont celles qui donnaient liea aux phénom mènes d'oscillation du plan d'eau, avant qu'il soit décidé de munir les piles d'arrière-becs efflles. On apercoit, en ava! des piles, les tourbillons alternes qui provoquent ces oscillations.

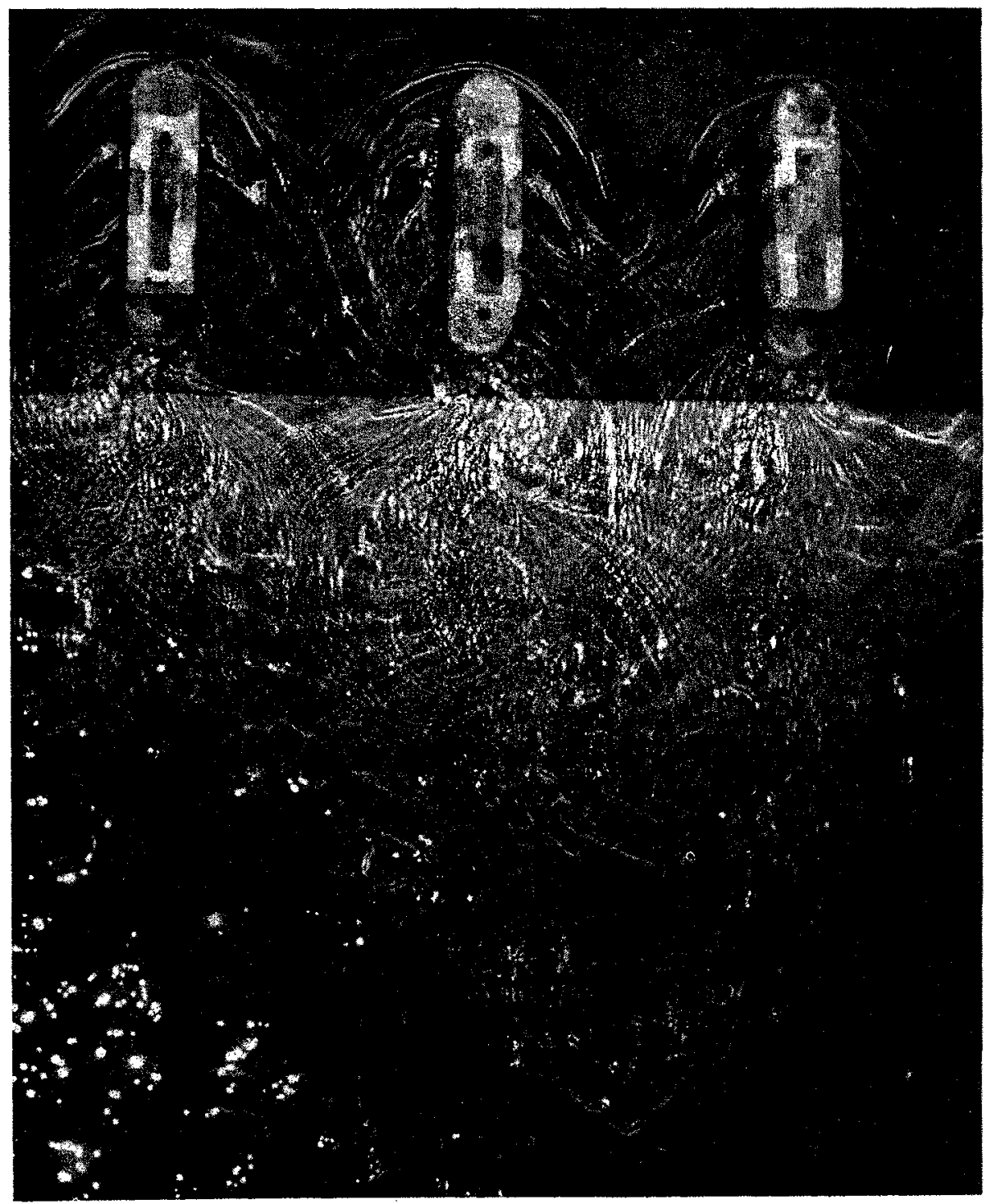

(Photo Labotatoire national a'H ydranliqut; 
trées sur deux ou trois passes, subissent en effet une dénivellation exceptionnelle à la traversée du barrage.

Pour ces différents cas, il a été nécessaire de determiner, par des essais sur fonds mobiles, la forme de radier qui conduit aux affouillements les moins importants. Des essais systématiques, portant sur la forne de l'auge (notamment longueur et profondeur) et l'utilisation éventuelle de dents favorisant la dissipation de l'énergie, ont été exécutés notamment pour les barrages de Rochemaure (le rocher, relativement tendre, est recouvert de $5 \mathrm{~m}$ de graviers), de Loriol $(1 \mathrm{~m}$ de graviers) et Pierre-Bénite (fonds affouillables sur une très grande profondeur) (4).

Cette forme optimale étant adoptée, des essais complémentaires permettent de définir la nature et l'étendue des protections (enrochements, gabions, etc.) propres à éviter l'érosion des berges ainsi que laffouillement du fond, au moins jusqu'à une certaine distance du barrage. D'au-

(4) Lorsque le faible débit réseryé est turbiné dans un groupe «bulbe» installé dans une des culées cas de Beauchastel et Pierre-Bénite), la forme de l'auge doit également tenir compte de cette condition particuliere tres essais, effectués sur des maquettes de radiers en plâtre, ont permis de localiser les zones d'usure préférentielles qui doivent être munies de revêtements spéciaux (granit notamment).

\subsubsection{ECOUlement des gRandes crues a TRA- VERS LES BARRAGES.}

L'écoulement des grandes crues (5) entre les piles d'un barrage donne lieu à des pertes de charge et à des dénivellations qui ont une grande importance pratique pour l'étude des lignes d'eau, mais qu'il est difficile d'apprécier sans le recours à des modèles réduits. De nombreux auteurs ont sans doute étudié les particularités de cet écoulement, mais les formules proposées s'appliquent à des ouvrages de formes très simples : piles de largeur constante (sans rainures pour les vannes ou batardeaux), radiers en général horizontaux (sans auge pour favoriser la dissipation de l'énergie), fond du lit très régulier en amont et en aval du radier et sans fosses dues à l'érosion. De plus, ces formules font intervenir

(5) Le tableau II et la figure 5 indiquent les prineipaux débits caractérístiques du Rhône à prendre en considération et les fréquences correspondantes.

\section{Tableau II. - Débits caractéristioues du Bas-Rhone}

Ce tablean donne, pour les fréquences caractéristiques, les valeurs du débit du fleuve à trois stations principales du Bas-Rhône: La Mulatière (en aval du confluent de la Saòne), Valence (en aval du confluent de l'Isère) et Joviac (au droit de l'aménagement de Montélimar. La station de Joviac est située sur le tronçon du fleuve courtcircuité par l'aménagement de Montélimar" les débits indiqués s'entendent dans l'état naturel (actuellement, ils correspondent au débit total «Rhône courtcircuité + dérivation»).

\begin{tabular}{|c|c|c|c|c|c|}
\hline & & & \multicolumn{3}{|c|}{ DÉBrTs $\mathrm{EN} \mathrm{m}^{3} / \mathrm{s}$} \\
\hline DÉSIGNATION & $\begin{array}{c}\text { PrOBABILTTE } \\
\text { TOTALE } \\
\mathrm{P}\end{array}$ & $\begin{array}{l}\text { HeVUENCE } \\
\text { (en } j / a n)\end{array}$ & $\begin{array}{c}\text { Mulatière( }) \\
\text { PK 0,7 } \\
1920-1959\end{array}$ & $\begin{array}{c}\text { Valence (**) } \\
\text { Pr } 109,7 \\
1920-1959\end{array}$ & $\begin{array}{c}\text { Joviac }(* *) \\
\text { PK } 158,4 \\
1920-1957\end{array}$ \\
\hline Plus faible valeur connue...... & $=$ & سي- & 200 & 300 & 360 \\
\hline Etiage (dépasse $355 \mathrm{j} / \mathrm{an}$ )..... & 0,027 & 355 & 320 & 470 & 510 \\
\hline Débit dépassé 9 mois/an...... & 0,25 & 274 & 565 & 820 & 870 \\
\hline Débit semi-permanent. . . . . . . & 0,5 & 182,6 & 820 & 1200 & 1250 \\
\hline Débit moyen $\ldots \ldots \ldots \ldots \ldots$ & - & - & 1021 & 1403 & 1487 \\
\hline Débit dépassé 3 mois $/ a n . . . .$. & 0,75 & 91 & 1270 & 1760 & 1880 \\
\hline Crue dépassée $10 \mathrm{j} / \mathrm{ar} \ldots \ldots \ldots$ & 0,973 & 10 & 2710 & 3450 & 3680 \\
\hline Crue annuelle $\ldots \ldots \ldots \ldots \ldots$ & 0,9973 & 1 & 3900 & 4950 & 5250 \\
\hline Crue décennale............. & 0,99973 & $10^{--1}$ & 5000 & 6100 & 6500 \\
\hline Plus forte crue connue........ & - & - & 6000 & 8300 & 8500 \\
\hline Crue millénaire............ & $1-2,74,10^{\cdots}$ & $10-3$ & 7500 & 9500 & 10000 \\
\hline
\end{tabular}

(') Pour l'aménagement de Pierre-Bénite.

$\left({ }^{*}\right)$ Pour l'aménagement de Beauchastel (usine de Beauchastel, barrage de Charmes).

(**) Pour les aménagements de Baix-Le Logis Neuf (usine du Logis Neuf, barrage de Loriol), Montélimar (usine de Châteauneuf, barrage de Rochemaure) et Donzère-Mondragon (usine de Bollène, barrage de Donzère). 


\section{COURBE DES FRÉQUENCES CUMULÉES}

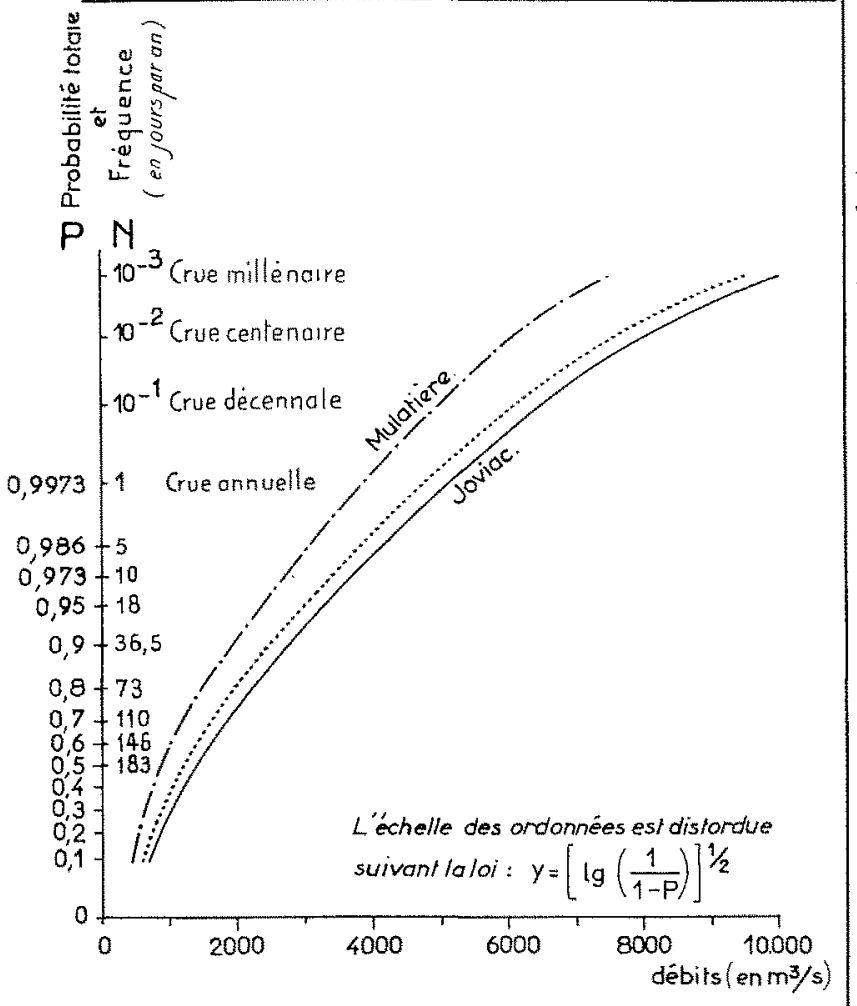

COURBE DES FREQQUENCES RELATIVES $P$ et portion de courbes des fréquences cumulées $P$

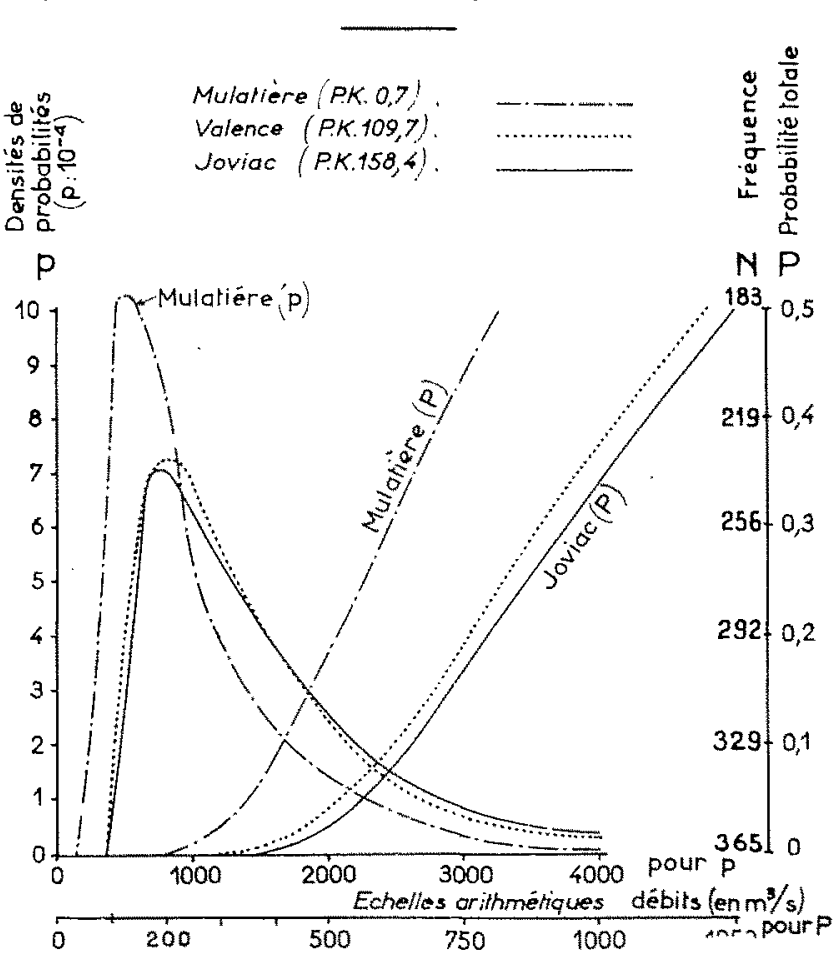

FIG. 5

Les graphiques ci-dessus illustrent et complètent le tableau II pour les trois stations considérées.

des paramètres qui ne sont pas toujours faciles à mesurér.

On sait que, si le barrage constitue une section de contrôle, le niveau aval $h_{v}$ n'a pas d'influence sur l'écoulement (voir notations sur figure 6). Le débit $Q$ est déterminé par le niveau amont $h_{m}$; sa valeur est donnée par la formule classique $\mathrm{Q}=\mu \mathrm{L}_{1} h_{m} \sqrt{2 g h_{m}}$, dans laquelle $\mathrm{L}_{1}$ désigne la largeur libre (somme des largeurs des pertuis) et $\mu$ est un coefficient de débit tenant compte des caractéristiques des pertuis (forme des piles et des radiers, rapport de l'épaisseur des piles à la largeur des pertuis, etc.).

La formule ne s'applique plus lorsque l'écoulement est gêné par le niveau aval. On est dans ce cas lorsque le rapport de submergence

$$
\left[s=\left(h_{v} / h_{m}\right)\right]
$$

dépasse 0,8 , ce qui est le cas de nos barrages lors des grandes crues (par exemple, pour la crue millénaire à Beauchastel :

$$
s=(9,6 / 10,35)=0,93) .
$$

M. G. de Marchi [10], notamment, a proposé d'utiliser dans ce cas une formule qui fait intervenir $h_{1}$ (épaisseur de la lame d'eau dans la sec- tion rétrécie) et $h_{m}$ (épaisseur homologue mesurée en amont). L'équation peut être mise sous la forme suivante: $\mathrm{Q}=m \mathrm{~L}_{1} h_{1} \sqrt{2 g\left(h_{m}-h_{1}\right)}$. La valeur du coefficient $m(6)$ dépend des dimensions des ouvrages ainsi que de $h_{m}$ et $h_{l}$. Or, nous avons indiqué que la forme de nos ouvrages est moins simple que celle étudiće par cet auteur, et comporte plusieurs rétrécissements et élargissements successif's. De plus, le niveau $\boldsymbol{h}_{1}$ est très difficile à mesurer dans la réalité et sur modèle réduit, tant en raison de l'instabilité de l'écoulement que de la forme compliquée du profil en long de la ligne d'eau à la traversée des ouvrages. Ce profil accuse notamment plusieurs mises en vitesses successives.

(6) Pour un ouvrage comportant un seuil faisant saillie d'une hauteur a par l'apport au fond du lit, $m$ aurait pour expression :

$$
m=\left[1-a \frac{\mathbf{L}_{1}^{2} h_{1}{ }^{2}}{\mathbf{L}_{m}{ }^{2}\left(h_{n}+a\right)^{2}}\right]^{-1 / 2} .
$$

$\alpha$ étant un coefficient numérique compris entre 1 et 1,1 . Eil remarquant que $h_{1} \mathrm{~L}_{1}=\mathrm{S}_{1}$ et $\mathrm{L}_{m}\left(h_{m}+a\right)=\mathrm{S}_{m}$ représentent les sections mouillées au droit de l'ouvrage et en amont, on peut aussi écrire :

$$
m=\left[1-a\left(\frac{S_{1}}{S_{m}}\right)^{2}\right]^{-1 / 2} .
$$




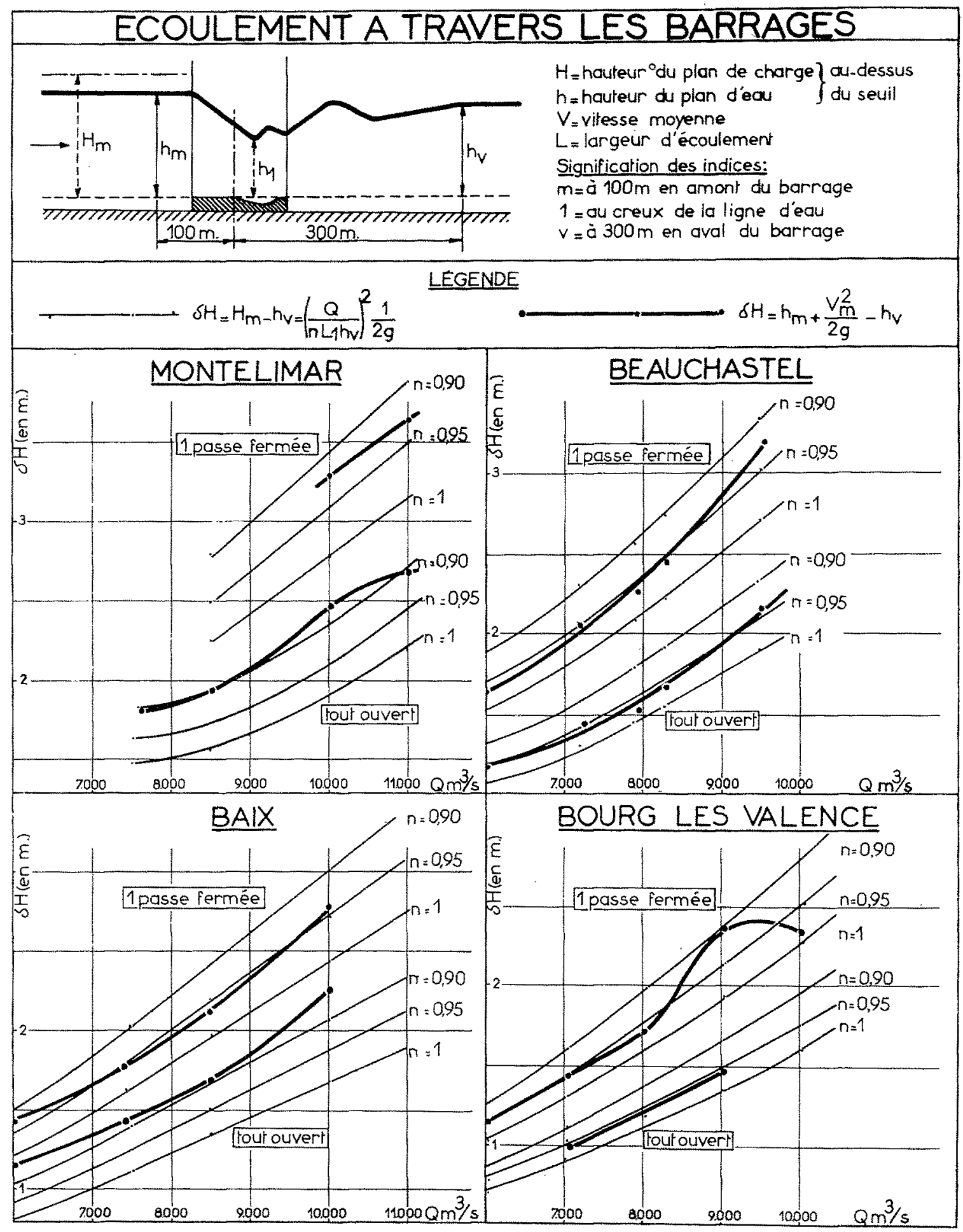

FIG. 6

Egovlement des grandes crues a travers les barrages.

Des mesures de niveaux et de vitesses ont été effectuées sur les modéles réduits de quatre aménagements du Bas-Rhône. Sur la base de ces mesures, on a calculé, pour différentes valeurs du débit traversant le barrage, les valeurs correspondantes du coefficient de débit $n$ figurant dans la formule utilisée pour nos études préliminaires. On voit que la valeur de ce coefficient est généralement très voisine de 0,95 . En utilisant cette valeur moyenne, l'approximation est très suffisante. 
Pour ces raisons, nous avons été amenés à étudier directement sur modèle réduit les pertes de charge pour quatre barrages du tiers central dont les dimensions principales, peu variables d'une chute à l'autre, sont indiquées sur la figure 1. Cette étude nous a conduit à rendre compte des phénomènes observés par la formule pratique suivante ( 7$): \mathrm{Q}=n \mathrm{~L}_{1} h_{v} \sqrt{2 g\left(\mathrm{H}_{m}-h_{v}\right)}$ qui nous a donné des résultats très acceptables ainsi qu'en témoigne la figure $6: n$ est en général compris entre 0,92 et 0,96 pour une gamme étendue de débits.

\subsection{Processus de construction des barrages.}

\subsubsection{GÉNÉRALITÉS.}

a) Mode de construction. - Les rives du Rhône étant fortement occupées par les installations humaines, les barrages de retenue sont généralement placés entièrement dans le lit mi-

(7) On peut considérer que cette formule dérive de l'expression classique suivante, où $k$ est un coefficient numérique caractérisant l'écoulement :

$$
Q=k L_{a} h_{1} \sqrt{2 g\left(H_{n t}-h_{1}\right)} \text {. }
$$

L'écoulement étant très voisin du régime critique, $h_{1}$ seraic lié à $h_{v}$ par la relation suivante, à la traversée d'un ouvrage comportant un rétrécissement unique de section (dû par exemple à des piles de pont ou à un seuil épais régulier) [7]:

$$
h_{v}^{2}+\frac{2 Q^{2}}{g h_{v} L_{v}^{2}}=h_{1}^{2}+\frac{2 Q^{2}}{g k h_{1} L_{v}^{2}}
$$

En posant $h_{x}=b h_{x}$, on voit que $b$ est racine de l'équalion $b^{3}-b(1+k \mathrm{~B})+\mathrm{B}=0$, dans laquelle on a posé :

$$
\mathrm{B}=\frac{2 \mathrm{Q}^{2}}{g k h_{r} \mathrm{~L}_{v}{ }^{2}}
$$

Le terme $B$ varie en fait dans nos ouvrages entre 0,5 et 0,25 (dans le système S.I.), lorsque $Q$ passe de la crue millénaire $\left(10000 \mathrm{~m}^{3} / \mathrm{s}\right)$ à la crue décennale $\left(6000 \mathrm{~m}^{3} / \mathrm{s}\right)$. Dans ce domaine, la racine utile $b$ varie très peu (de 0,97 à 0,985 ), ce qui est de nature à justifier la substitution de $h_{1}$ par $h_{v}$ que nous avons faite.

Avec les formes relativement compliquées des radiers et du fond du lit au voisinage des barrages du BasRhône, on constate que la cote minimale ne se situe pas toujours au droit du radier, mais souvent légèrement en aval, et que, dans la plupart des cas, la hauteur d'eau minimale sur le radier est en effet peu inférieure à $h_{v}$.

En toute rigueur, les hauteurs $\mathrm{H}_{m}, \mathrm{H}_{v}$ et $h_{v}$ qui interviennent dans les formules classiques devraient être mesurées au roisinage immédiat de l'ouvrage, et non à une certaine distance comme on est obligé de le faire en pratique ef comme nous l'avons fait dans la formule ci-dessus. Les diverses simplifications qui ont été faites expliquent sans doute les variations, d'ailleurs faibles, du coefficient $n$ qui y figure, et dont notre graphique rend compte. L'expression de la perte de charge à la traversée du barrage (mesurée sur une distance de $400 \mathrm{~m}$, soit environ deux fois la largeur du lit), serait :

$$
\mathrm{H}_{m}-\mathrm{H}_{v}=\frac{\mathrm{V}_{v}{ }^{2}}{2 g}\left[\frac{1}{n^{2}}\left(\frac{\mathrm{L}_{m}}{\mathrm{~L}_{1}}\right)^{2}-1\right]
$$

$V_{v}$ désignant la vitesse moyenne en aval $\mathrm{Q} / \mathrm{L}_{v} H_{v}$. Le terme entre crochets prend la valeur 0,67 pour les valeurs les plus fréquentes de $n$ et de $L_{\tau} / L_{1}$.

$$
\left(n=0,95 ;\left(L_{v} / L_{1}=1,22\right) .\right.
$$

neur, lui-même creusé dans les alluvions affouillables déposées par le fleuve (Donzère, Montélimar, Baix-Le Logis Neuf).

L'ouvrage est entrepris par tronçons successifs réalisés à l'abri de batardeaux qui obstruent par conséquent une partie des lits mineur et majeur (fig. 7). Grâce à une étude détaillée sur modèle réduit, le nombre des phàses de construction (comportant chacune l'édification et la démolition d'un important batardeau) qui était de cinq à Donzère [8], a pu être ramené à quatre à Montélimar [3 et 4 ] et à Baix-Le Logis Neuf pour un ouvrage construit entièrement dans le lit mineur du fleuve.

Pour les barrages plus récents, les circonstances ont permis de décaler l'ouvrage par rapport au lit mineur, ce qui facilite grandement la construction du barrage proprement dit: à Beauchastel, où il a été décalé de $45 \mathrm{~m}$ sur rive droite, le nombre des phases a été ramené à trois. A Pierre-Bénite, où l'ouvrage est placé sur un chenal entièrement artificiel creusé dans le lit majeur, une seule phase de travaux sera nécessaire pour la construction complète de l'ouvrage.

b) Ecoulement des crues. - La construction des barrages doit évidemment ètre conduite de manière à ne gêner ni l'écoulement des crues, ni l'exercice de la navigation.

Pour remplir ces deux conditions, l'obstruction doit être compensée par un débouché supplémentaire. Ce débouché pourrail sans doute être constitué, si le lit est affouillable, par l'approfondissement naturel du lit qui survient après le passage des crues. Mais l'érosion n'est pas immédiate, d'où il peut résulter un risque temporaire de submersion pour les riverains de l'amont, et une gêne momentanće pour les bateaux en raison des survitesses qu'ils auront à franchir. Au surplus, les matériaux mobilisés vont souvent se déposer en aval sur un seuil qui risque de ne plus assurer le tirant d'eau nécessaire en étiage. Il y a donc intérêt à prévoir le débouché complémentaire artificiel par un dragage préventif ou par une dérivation provisoire.

c) Le passage de la navigation au droit du chantier impose en outre les conditions suivantes :

-- le chenal de navigation doit conserver un tracé aussi direct que possible. Il est même souhaitable (l'expérience de Montélimar l'a montré) que la position du chenal reste la même pendant toute la durée des travaux; mais cette dernière condition n'est pas toujours réalisable;

- la vitesse du courant et la pente du plan d'eau ne doivent pas être exagérément accrues et rester suffisamment loin de l'écoulement critique pour éviter les ondula- 


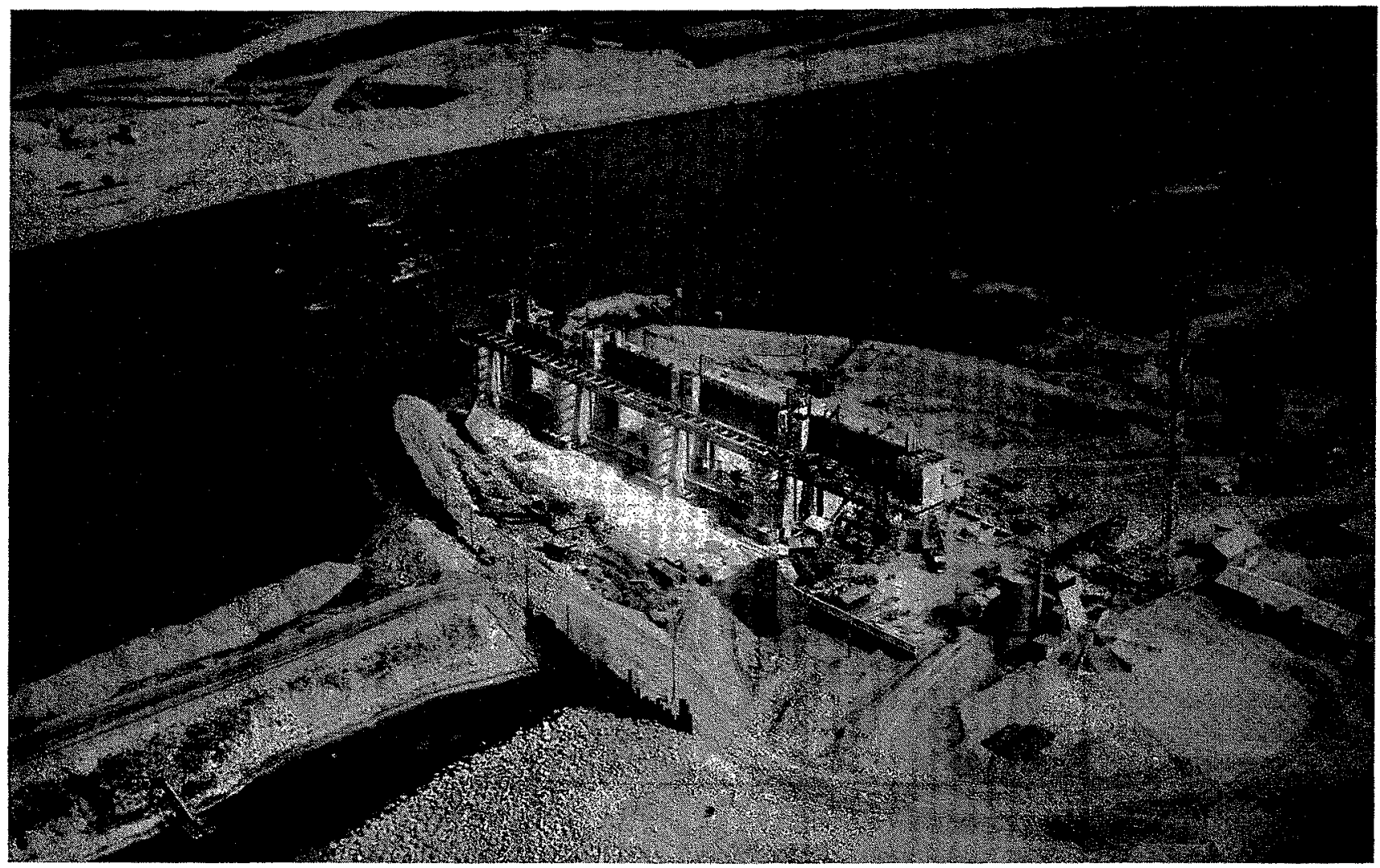

Fig. 7

(Photo Stultos Villetrbanatis.)

Construction DU BarRace on Charmes (aménagement de Beauchastel).

Vue acrienne du chantier pendant la construction des quatre passes rive droite. Le lit du Rhône a été élargi sur sa rive gauche pour constituer une pseudo-dérivation provisoire. Les remous visibles dans le lit du Rhône sont provoqués par des balises signalant la présence d'un seuil rocheux naturel (seuil des Pierres de Charmes); le chenal navigable passe entre ce seuil et l'enceinte de construction.

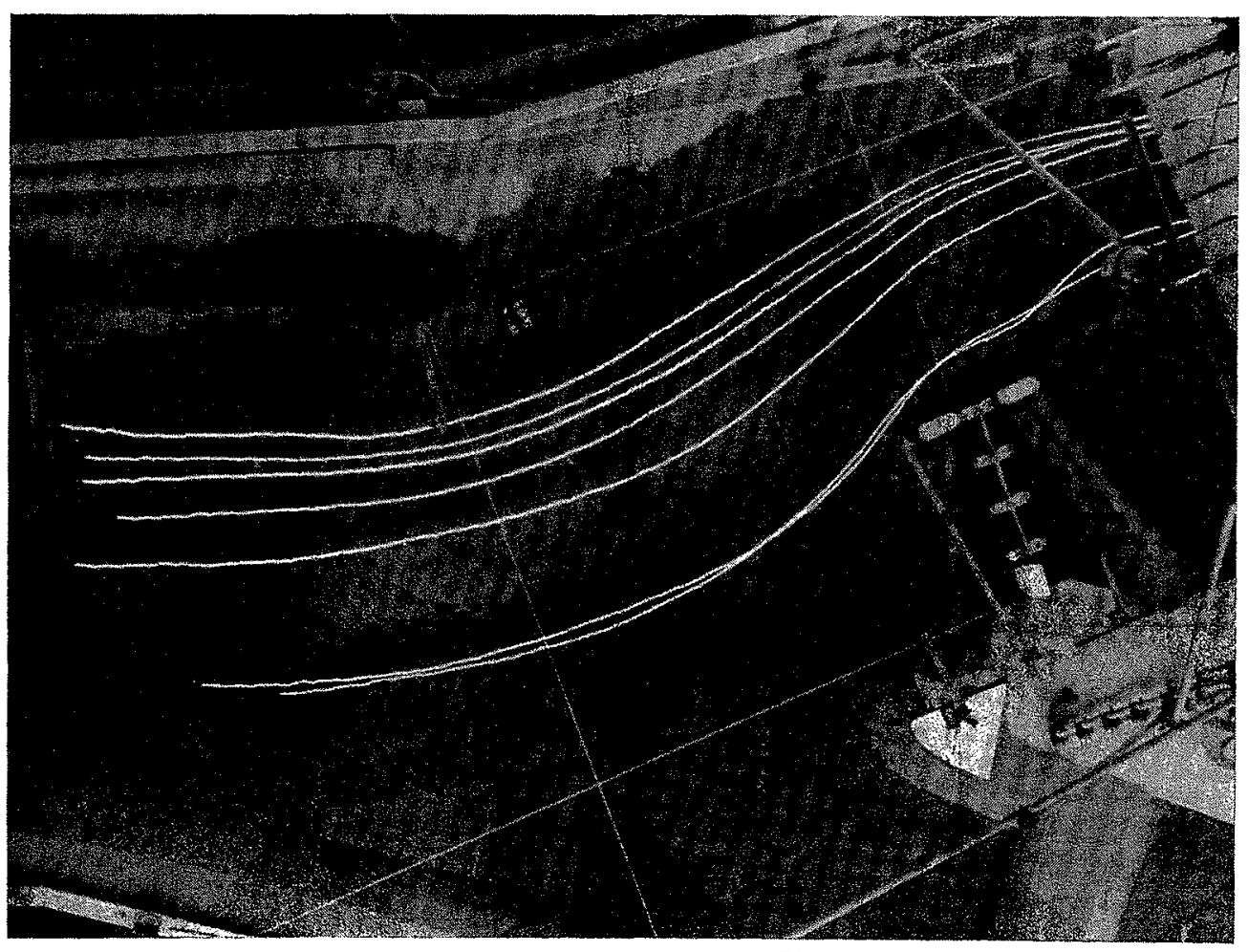

(Photo Laboratoire national $\left.d^{x} H y d r a n l i q u t e.\right)$
FIG. 8

ECOVLEMENT DUं RHONe a La TRAVERSÉE DU CHANTIER DU barrage de Charmes (vue partielle du modèle réduit).

Visualisation des lignes de courant dans le Rhône au droit du chantier pendant la construction des quatre passes rive droite. Les photos de ce genre doivent être réalisées dans une obscurité partielle, avec utilisation de flotteurs lumineux. 
tions. En raison de l'absence d'une dérivation provisoire à Donzère, oủ les études hydrauliques furent moins complètes, la navigation a rencontré de sérieuses difficultés à la traversée du chantier. Si le chantier se trouve au droit d'un passage déjà naturellement difficile (cas de Beauchastel), il convient même d'éviter tout accroissement de la vitesse et de la pente;

- le courant dans le chenal navigable doit être bien dirigé; il faut éviter absolument la formation de courants traversiers qui risqueraient d'entrainer les bateaux vers des obstacles et notamment vers les batardeaux (fig. 8);

- le mouillage dans le chenal doit rester suf- fisant en basses eaux; pour cela, il convient que le transit du débit solide s'effectue sans formation de dépôts dans le chenal navigable.

\subsubsection{EsSAIS SUR MODÈLE RÉDUIT.}

Toutes les conditions indiquées ci-dessus imposent de définir à l'avance un processus précis pour la construction du barrage en différentes phases de travaux. Cette définition ne peut s'effectuer complètement par des considérations de simple bon sens, ni même par une étude théorique, si poussée soit-elle. Il est nécessaire de recourir à des essais très détaillés sur un modèle réduit (fig. 9); l'échelle de ce modèle doit être suffisamment grande $(1 / 100$, on même $1 / 60$ ), et sans distorsion, afin que les phéno-
Fig. 9

VUE D'ENSEMBLE DU YODÈLE RÉDUTT POUR L'ÉTUDE DU BARRAGE ET DE LA PRISE D'EAX DE Beavchastel.

A l'arrière-plan: le barrage en cours de construction et l'entrée du canal d'amenée (on aperçoit l'exutoire par lequel se deverse tans le canal un petit torrent : l'Embroye).

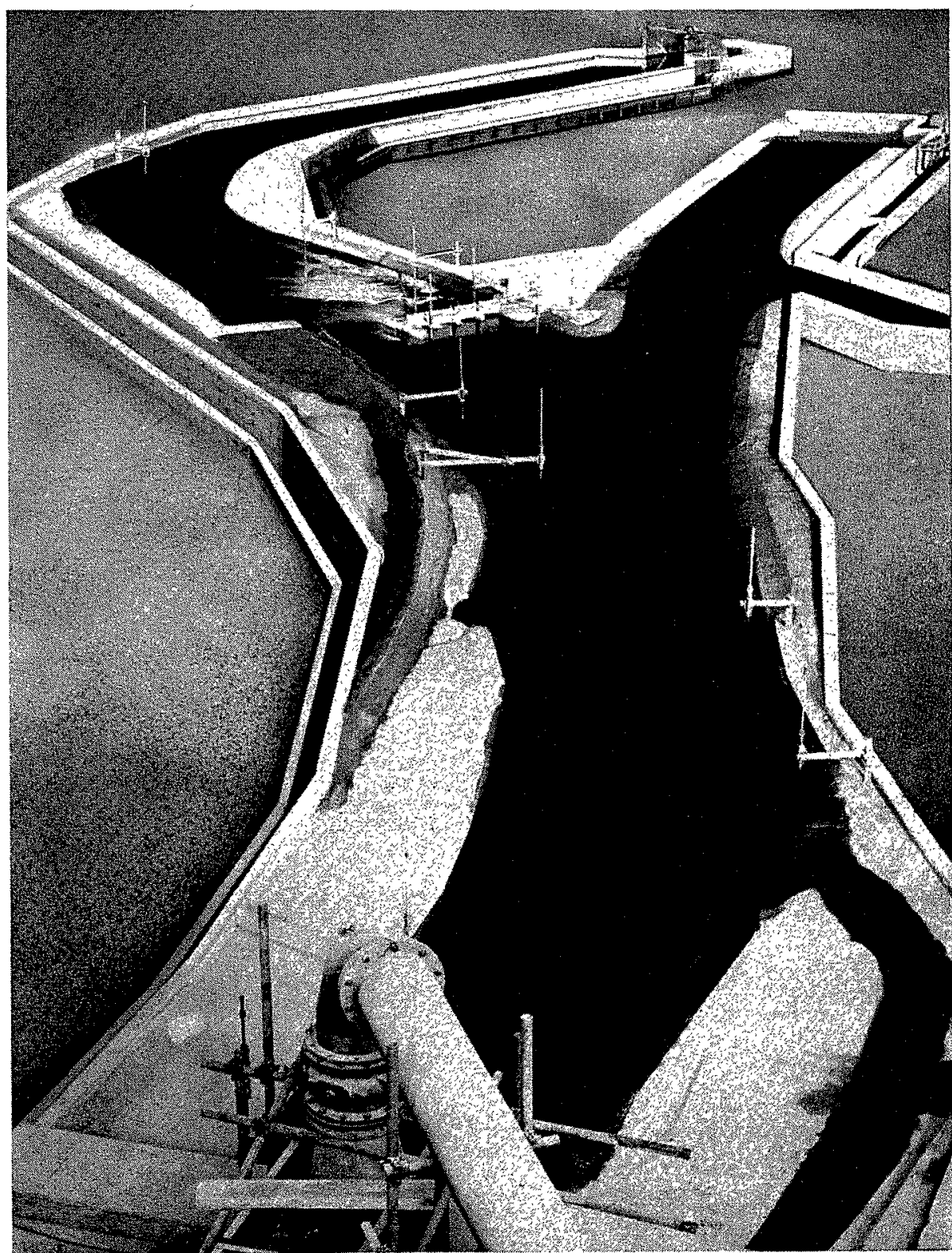

(Photo Laboratoive national d'Hydraulique.) 
mènes de charriage se produisent avec le maximum de similitude (8).

L'étude sur ce modèle réduit comporte une première série d'essais rapides permettant de définir les grandes lignes du processus de construction, les essais relatifs à chacune des phases de travaux étant repris par la suite d'une manière plus détaillée.

Nous allons maintenant indiquer à quelles conclusions d'ordre général ces essais nous ont permis d'aboutir en ce qui concerne le problème des dérivations provisoires et celui de la coupure du fleuve.

\section{a) Conditions d'établissement de la dérivation provisoire :}

Implantation. -- Dans la mesure du possible, on ntilise les particularités du site pour implanter au mieux la ou les dérivations provisoires: présence d'une lône naturelle (à Montélimar ou à Pierre Bénite), du chenal nécessaire à la dérivation d'un affluent (cas du Lavaizon à Montélimar), et on tient compte de la possibilité de draguer les fonds ou de rescinder les berges (Beauchastel et Pierre-Bénite).

Certains principes généraux concernant l'établissement de ces dérivations provisoires dans nos aménagements mixtes ont pu être mis en évidence à la suite des essais :

- une dérivation navigable est difficilement viable. En effet, une telle dérivation doit avoir une grande largeur et un plafond assez bas; elle dérive alors une part importante du débit; par ailleurs, pour être accessible à la navigation, la dérivation doit avoir une entrée et une sortie formant des angles aussi faibles que possible avec la direction du fleuve; il en résulte un accroissement considérable de la section d'écoulement, qui provoque des dépôts de gravier dans le chenal navigable et un abaissement des lignes d'eau. L'importance des dépôts et la rapidité de leur formation sont telles qu'on ne peut envisager d'entretenir le chenal par dragage (essais de Montélimar et Baix);

- on n'a pas intérêt à utilliser une partie du futur canal comme dérivation provisoire. En

(8) Si on veut pouvoir rendre compte fidèlement du cheminement du debit solide, il est indispensable de représenter avec exactitude tous les détails du lit mineur (avec ses digues, ses epis et protections d'enrochements) et ceux du lit majeur (avee ses obstacles et sa rugosité).

Le modele peut permettre d'attirer l'attention sur des phénoménes qui ont échappé à l'attention des services intéressés. Ainsi, le modèle de la prise de Baix-Le Logis Neuf nous avait conduit à représenter les piles du vieux pont du Pouzin, situé à $2 \mathrm{~km}$ du barrage, avec leurs pro. tections en enrochements. Dès sa mise en eau, le modèle a montré qu'il devait exister en aval de ces piles des affouillements très importants avec des fosses de $13 \mathrm{~m}$ de profondeur sous le niveau moyen du lit. Nous avons alors procédé à des sondages qui ont confirmé l'existence et la profondeur de ces fosses ignorées de tous. effet, le canal est implanté à l'extérieur d'une courbe (même si celle-ci est peu marquée) où se sifuent les plus grandes profondeurs, et par conséquent le chenal navigable. Les dépôts inhérents à toute dérivation provisoire se forment alors dans ce chenal; à l'aval, la restitution de la d'érivation provisoire crée dans ce même chenal des courants traversiers très gènants pour les bateaux.

La dérivation provisoire doit donc se situer du côté opposé au chenal navigable et, par conséquent, au futur canal (fig. 10). Les atterrissements qui se forment éventuellement sont situés en dehors du chenal navigable. Si la dérivation s'engrave, il s'ensuit une légère augmentation des vitesses qui peut être supprimée le cas échéant par des dragages d'entretien ne gênant pas alors la navigation (essais de Baix).

Débit dérivé. - L'étude des dérivations provisoires de nos aménagements mixtes (notamment Baix-Le Logis Neuf et Pierre-Bénite) a montré qu'il convient de limiter le débit ainsi dérivé, non seulement en eaux moyennes (pour accrồtre les vitesses dans le chenal navigable et évitex ainsi la formation des dépôts), mais également en basses eaux (pour éviter un abaissement des lignes d'eau d'étiage qui réduirait le mouillage); au contraire, lors des crues, la dérivation provisoire doit écouler un débit suffisant pour que les vitesses ne deviennent pas exagérées dans le chenal navigable. Afin de concilier ces deux impératifs, la meilleure solution consiste à barrer la dérivation provisoire par un seuil déversant qui supprime l'écoulement en basses eaux et le réduit en eaux moyennes, sans pour cela le réduire notablement lors des crues.

Par ailleurs, la présence du chantier modifie généralement les conditions de transit du débit solide, et il faut aussi éviter que des atterrissements mal placés (soit dans le lit, soit dans la dérivation provisoire) ne provoquent une réduction de la section d'écoulement.

Le contrôle du débit de la dérivation provisoire doit avoir pour effet d'éviter tout soutirage d'eau lorsque le dẻbit $Q$ du Rhône est voisin du débit $Q_{c}$ de commencement de charriage. S'il n'en était pas ainsi, les dépôts, au lieu de se former uniquement au voisinage de la dérivation (sans gêne pour la navigation) s'effectueraient sur toute la largeur du Rhône (donc aussi dans le chenal navigable).

\section{b) Coupure du Rhòne à l'avancement:}

Sauf à Pierre-Bénite, ò̀ le problème sera résolu d'une manière un peu différente (9), l'opération de la coupure du fleuve intervient lorsque le barrage est aux deux tiers construit (quatre passes sur six). Il faut alors couper le Rhône

(9) Mais toujours à l'avancement. 


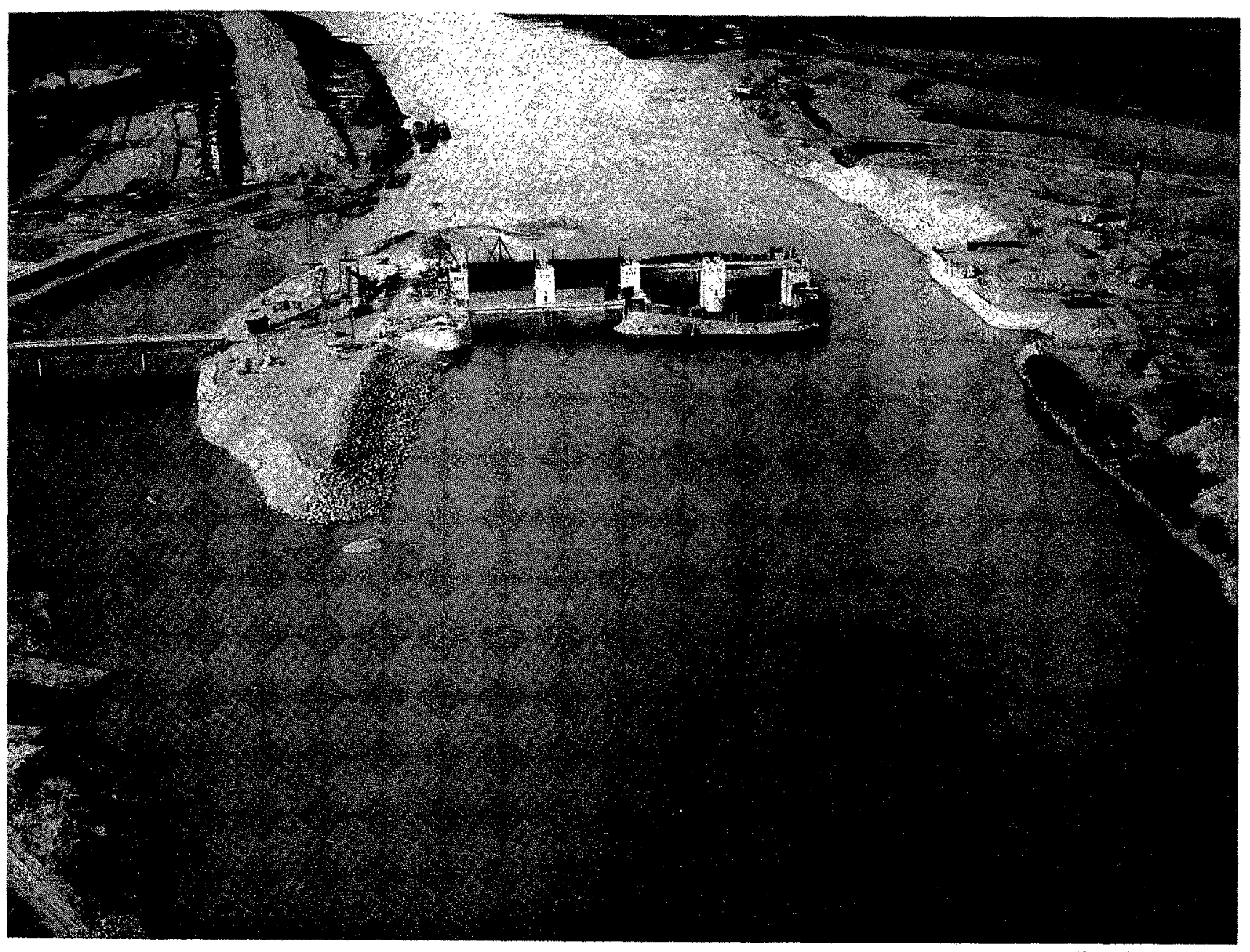

Fis. 10

(Photo Sitdios Villeurbanunis.)

Construction du barrage de Loriol (aménagement de Baix-Le Logis Neuf).

Le chantier du barrage au cours de la construction des deux passes centrates (vue aérienne prise en direction de l'amont). Du côté gauche de la photo (rive droite), on voit une partie de la dérivation provisoire, bartée par un seuil submersible. Du cóté droit (rive ganche), le musoir d'entrée, la culée! du barrage et ses murs en aile sont déjà construits. Les vannes sont en cours de montage dans les deux passes rive droite dont le génie civil est terminé.

au droit des deux dernières passes restant à construire, pour mettre l'aménagement en retenue et permettre à la navigation d'emprunter le canal définitif. Pendant cette opération la navigation ne peut plus passer dans le Rhône, et en général elle ne peut pas encore passer dans le canal (en raison du mouillage insuffisant au seuil d'entrée et à la tête amont de l'écluse). L'exercice de la navigation étant interrompu, il convient d'effectuer la coupure le plus rapidement possible.

Deux méthodes principales peuvent a priori être utilisées pour la réalisation de la coupure: - la méthode par tranches horizontales, préconisée par Isbach et utilisée pour la coupure de Génissiat; si elle conduit peut-être à une plus grande économie en matériaux, cette méthode ne peut être appliquée que si l'on dispose d'un pont accessible aux gros véhicules de terrassements pour répandre les matériaux de façon très égale sur toute la longueur de la coupure. Cette condition ne pouvant être réalisée de façon économique sur les barrages du Bas-Rhône, on a renoncé à celte méthode;

- la méthode d̀ l'avancement, où l'on édifie une digue par déversement de matériaux graveleux dans le fleuve en partant d'une des rives de la brèche.

C'est cette dernière méthode qui a été adoptée sur les chantiers de barrage du Bas-Rhône; la coupure s'effectue donc par édification d'une digue au droit des deux passes restant à construire, 
cette digue constituant ultérieurement l'un des batardeaux provisoires (amont ou aval) pour la dernière phase du chantier.

Au fur et à mesure de l'avancement de la digue, le chenal se rétrécit; la pente et la vitesse du courant s'accroissent, car le niveau en amont n'est pas encore suffisant pour permettre à l'eau de passer dans les quatre passes déjà construites. Si l'on déversait des matériaux trop fins, il serait impossible de réaliser la fermeture de la digue; en effet, vers la fin de lopération, ces matériaux seraient entrainés par le courant et iraient se déposer plus en aval. Il est done nécessaire de déverser en même temps des blocs de carrière qui restent plus stables dans le courant. La proportion des blocs à utiliser dépend d'ailleurs du débit du Rhône au moment de l'opération, ainsi que de la cadence de déversement possible selon les caractéristiques des engins de chantier utilisés.

Pour préparer l'opération, qui ne doit comporter aucune incertitude, il est nécessaire d'effectuer des essais très complets sur le modèle réduit, en considérant plusieurs hypothèses de débit du fleuve. Ces essais permettent de déterminer le meilleur tracé à donner à la digue de coupure et la nature des matériaux à utiliser pour réaliser l'opération dans le délai le plus réduit.

D'une manière générale, les essais ont permis de déterminer, en fonction du débit du Rhône et de la cadence de déversement des matériaux, le pourcentage minimal de matériaux chers (enrochements ou déchets de carrière) à mélanger au tout-venant pour permettre la fermeture, ainsi que le temps nécessaire à cette fermeture : pour un débit du Rhône donné, la durée de la coupure est d'autant plus courte que ce pourcentage est plus grand, et la proportion d'enrochements croît évidemment avec le débit qu'il faut couper.

Lorsque la passe réservée à la coupure se trouve en bordure de la berge, où les vitesses sont modérées, des essais comparatifs ont montré qu'il y avait intérêt à commencer par exécuter le batardeau amont (Montélimar et Beauchastel). Au fur et à mesure de sa constitution, le massif vient en effet s'appuyer sur la pile en rivière et restreindre le débit résiduel (fig. 11).

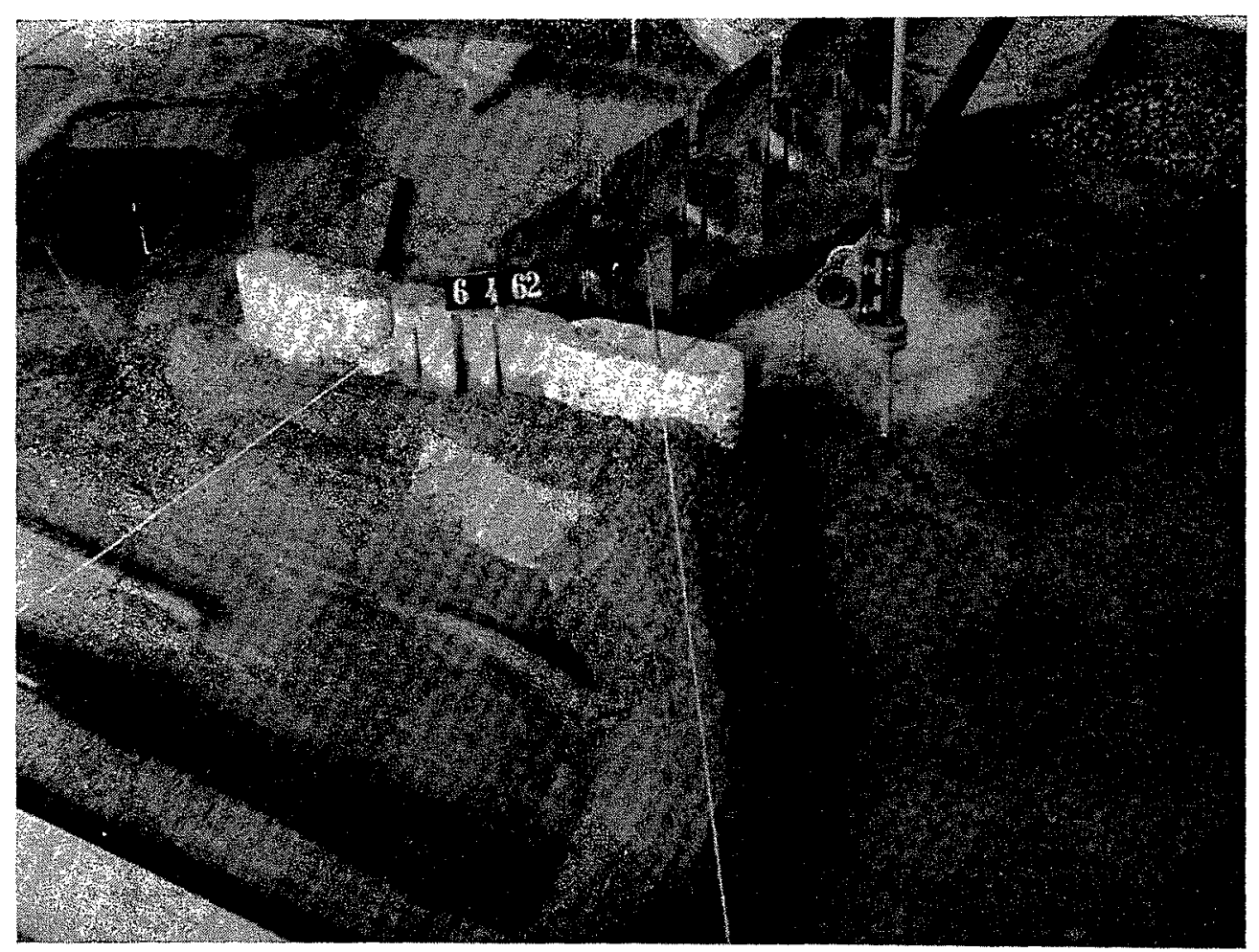

FiG. 11

(Photo Laboratoire national d'Hydraulique.)

UNE COUPURE DU RHONE

(étude sur modèle réduit).

Etude de la coupure rapide du Rhône, après construction des quatre passes rive droite du barrage de Charmes. La photo représente notamment la digue de coupure après la fin de l'opération; cette digue, partant de la berge rive gauche, se referme sur Jes cellules en palplanches qui protégeaient la pile $\mathrm{V}$ pendant sa construction. Le modèle a été vidangé, pour procéder au relevé des fonds; on apercoit les matériaux que le courant a étalés vers l'aval au cours de l'opération de coupure. 

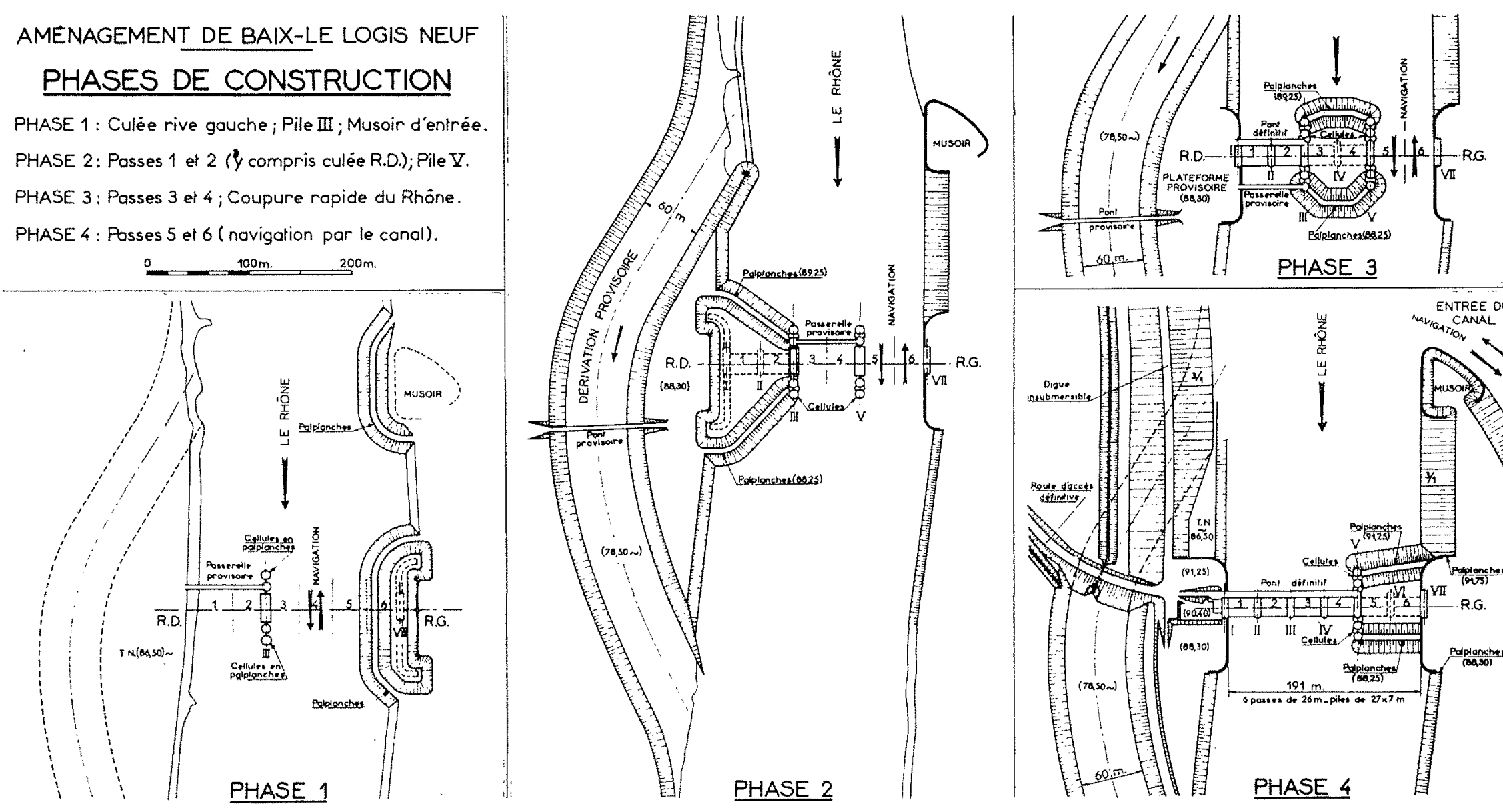

FIG. 12

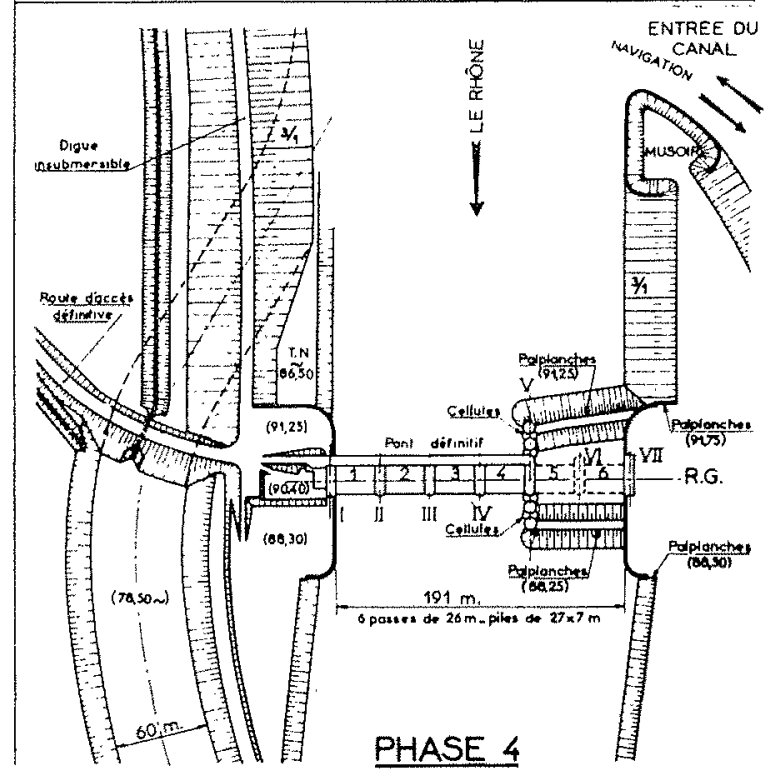

UN EXEMPLE DV PROCESSYS DE CONSTRUGTION DES RARRAGES EN RTYLRE (barrage de Loriol).

Phașes principales de la construction, dérivation provisoire et enceintes de protection. 
Lorsque la passe est siluée au milieu de la rivière et limitée par des piles ou cellules de palplanches entre lesquelles la vitesse est élevée, les essais comparatifs montrent, au contraire, qu'il vaut mieux commencer par le batardeau aval. La vitesse du courant est en effet plus faible en aval des cellules qu'au droit du rétrécissement et la proportion de matériaux entraînés au loin par le courant reste par conséquent plus modeste (Baix).

\section{c) Essais propre à chaque chate :}

Pour donner une idée plus précise des études hydrauliques lices à la définition du mode d'exécution des barrages, et pour éviter de rester dans les généralités, nous citerons quelques exemples choisis dans l'étude du barrage de Loriol (aménagement de Baix-Le Logis Neuf). Nous donnerons ensuite un aperçu des études hydrauliques entreprises pour les deux ouvrages actuellement en construction.

\subsubsection{PROCESSUS DE CONSTRUCTION DU BARRAGE} DE LORIOL.

a) Généralité et passage de la navigation:

Pour des motifs techniques et économiques, le nombre des passes du barrage a été fixé à six; d'autre part, les conditions hydrauliques et topographiques ont conduit à placer l'ouvrage entièrement dans le lit mineur du fleuve. Celui-ci n'est séparé du fond rocheux que par une mince couche de sables et graviers (de 0,5 à $1,5 \mathrm{~m}$ d'épaisseur). Afin de ne pas trop obstruer le lit, il paraissait judicieux de construire louvrage par tiers (trois phases de deux passes chacune) à l'abri de batardeaux (fig. 12).

Il importe de remarquer tout d'abord que pendant la construction des deux dernières passes, la navigation ne peut plus passer dans le Rhône; en effet, dans les quatre passes déjà construites, la largeur libre et le tirant d'air sont insuffisants. Il faut done que la navigation emprunte soit la dérivation provisoire, soit le canal de dérivation définitif :

- la premiere hypothèse aurait été la bienvenue, car elle aurait permis de construire entièrem ment le barrage avant la mise en retenue. Mais, nous l'avons dit, des essais détaillés sur modèle réduit ont conduit à éliminer cette solution;

- il faut donc se résoudre à la seconde hypothèse; les deux dernières passes du barrage ne peuvent être construites qu'après l'achèvement des autres ouvrages et la mise en retenue de l'aménagement. II en résulte certains inconvénients; le plus grave est que, pendant la construction des deux dernières passes, le débouché disponible pour l'écoulement des crues se trouve réduit: Ies deux tiers seulement du barrage sont disponibles, la dérivation provisoire est hors service pour permettre la mise en retenue, le débit dérivé est limité car tous les groupes hydroélectriques ne sont pas encore en ordre de marche. Pour réduire les risques, il convient donc que la durée de cette dernière phase ne soit pas trop longue.

Cette question préjudicielle étant réglée, des essais rapides ont confirmé la possibilité de construire le barrage en trois séries de deux passes, la dernière série étant réalisée après la mise en eau de l'aménagement; le débouché complémentaire nécessaire pendant la réalisation des deux premières séries peut être procuré par une dérivation provisoire de dimensions raisonnables.

\section{b) Implantation de la derivation provisoire:}

Il pouvait être tentant de placer cette dérivation en partie sur le tracé du canal définitif, afin de réduire le volume des terrassements (creusement, puis remblaiement ultérieur de la dérivation provisoire). Mais, comme nous l'avons dit, les essais ont montré que cette solution ne convenait pas. On a donc placé la dérivation provisoire sur la rive opposée à l'entrée du canal définitif, à savoir la rive droite.

\section{c) Phases de construction :}

Les batardeaux provisoires constituant chaque enceinte de construction se referment sur les rives du fleuve et sur certaines piles du barrage ódifiées préalablement sur caissons à l'air comprimé.

Le barrage est construit en allant de la rive droite à la rive gauche. Afin de réduire le délai d'exécution de la dernière phase (après la mise en eau de l'aménagement), il convient de construire à l'avance la culée rive gauche et les murs en aile qui la prolongent. Une première phase de travaux comporte donc la construction de ces ouvrages, ainsi que celle du musoir d'entrée et de la pile III (les passes ainsi que les piles et les culées sont numérotées à partir de la rive droite); en même temps, on creuse la dérivation provisoire,

La phase $\mathrm{n}^{\circ} 2$ comporte la construction des derx passes de rive droite, la phase $n^{\circ} 3$ celle des deux passes centrales; enfin, la phase $n^{\circ} 4$ concerne la construction des deux passes de rive gauche. Pendant les phases $n^{\circ} 1,2$ et 3 , la navigation continue d'emprunter le chenal navigable naturel, qui longe la rive gauche du fleuve; à partir de la phase $\mathbf{n}^{\circ} 4$, elle emprunte définitivement le canal usinier.

Des essais détaillés sur modèle réduit sont effectués pour chacune des phases, afin de vérifier les conditions de navigation, de prévoir l'évo. lution des fonds et de définir les protections des batardeaux provisoires. Ces essais se poursuivent 
pendant toute la durée du chantier, pour définir les mesures à prendre dans certaines circonstances qui n'avaient pu être prévues à l'origine des travaux. Nous donnons ci-après ( $\S \mathrm{e}$ ) un exemple de ces essais de circonstance.

\section{d) Coupure rapide du Rhône:}

Cette coupure s'est réalisée sans aucune difficulté, dans les conditions prévues sur le modèle. Le débit $\mathrm{du}$ fleuve était modeste (environ $\left.900 \mathrm{~m}^{3} / \mathrm{s}\right)$; la digue a pu être constituée par déversement de $12000 \mathrm{~m}^{3}$ de matériaux de carrière fins, sans incorporation de gros blocs; l'opération a duré seulement $16 \mathrm{~h}$.

Mais nous rappellerons le cas du chantier de Rochemaure (chute de Montélimar) [3 et 4], pour lequel le débit du Rhône pendant la coupure est passée de 1100 à $2200 \mathrm{~m}^{3} / \mathrm{s}$ environ; il a été nécessaire de déverser plus de $20000 \mathrm{~m}^{3}$ de matériaux fins auxquels ont été ajoutés, pendant la dernière partie de l'opération, 3000 à 4000 tonnes d'enrochements dont le poids unitaire variait de 200 à $500 \mathrm{~kg}$ environ; l'opération a duré au total $92 \mathrm{~h}$.

e) Exemple d'essais concernant les mouvements de fond du lit du Rhône:

Voici maintenant un exemple des mouvements de fond du lit susceptibles de se produire au cours des travaux, ainsi que des essais complémentaires sur modèle auxquels ils peuvent donner lieu.

Nous avons indiqué qu'au cours de la phase $n^{*} 1$ des travaux, il a été procédé au creusement de la dérivation provisoire contournant le chantier du barrage sur la rive droite. Cette dérivation devait être mise en service au moment de l'édification des batardeaux destinés à permettre la construction des passes nos 1 et 2 (phase $n^{\circ} 2$ ).

En fait, la dérivation a été ouverte le 6 juin 1958 ; mais, pour des raisons diverses de chantier, la construction des batardeaux n'a pu être entreprise immédiatement. Vers la fin juin, des dépôts de graviers étaient constatés dans le chenal navigable. Il a alors été demandé au laboratoire d'essais :

- de vérifier si le modèle rendait bien compte des atterrissements observés, compte tenu de l'état du chantier et des débits du Rhône;

- de définir des mesures propres à permettre le dégravement du chenal et son maintien ultérieur.

Les essais ont montré que les dépôts résultaient de l'accroissement de la section d'écoulement, provoqué par l'ouverture de la dérivation provisoire sans fermeture concomitante des passes nos 1 et 2. En outre, le débit du Rhône était resté un peu supérieur au seuil de charriage ( $1500 \mathrm{~m}^{3} / \mathrm{s}$ environ) pendant la plus grande partie du mois. Pour obtenir le dégravement du chenal, et assurer son maintien ultérieur, il convenait de fermer au plus vite les passes nos 1 et 2 et de constituer dans la dérivation provisoire un seuil qui supprime tout écoulement dans cette dérivation pour les débits du Rhòne inférieurs à $2000 \mathrm{~m}^{3} / \mathrm{s}$ environ.

Pendant la durée des essais complémentaires indiqués ci-dessus, l'engravement du chenal s'était poursuivi. Il n'en résultait pas d'inconvénient immédiat pour la navigation; en effet, le débit du Rhône restant assez soutenu, le mouillage était suffisant; mais, si un étiage était survenu, la navigation eût pu se trouver complètement arrêtée.

Le 12 juillet, la situation des fonds était celle représentée sur le croquis $A$ de la figure 13. Les zones dont l'altitude était supérieure à $(80) \mathrm{NGF}$ risquaient de provoquer l'arrêt de la navigation en basses eaux; elles sont représentées sur le croquis par des hachures.

Vers fin juillet, le programme des travaux permettait d'effectuer la coupure des passes nos 1 et 2. If en résultait un début de régression des engravements : le croquis $B$ représente la situation au 6 août. Enfin, vers le 15 août, une baisse de débit du fleuve permettait de réaliser le seuil déversant dans la dérivation provisoire. La régression des engravements s'accélérait alors; dès le début de septembre, une situation convenable se trouvait rétablie; le croquis C représente les fonds obtenus le 11 septembre.

Cependant, au début d'octobre, une crue subite emportait le seuil déversant constitué dans la dérivation provisoire. Après cette crue, les engravements progressaient de nouveau; la situation obtenue au 29 octobre est représentée sur le croquis $\mathrm{D}$; celle au 26 novembre est représentée stur le croquis $\mathbf{E}$.

Il était alors demandé au laboratoire d'essais :

- de vérifier la représentativité du modèle, en reproduisant les débits constatés sur le chantier entre le 29 octobre et le 26 novembre, la situation des fonds à l'origine étant bien entendu celle relevée in situ le 29 octobre (croquis D);

- d'examiner s'il était nécessaire de rétablir le seuil dans la dérivation provisoire, ou bien s'il serait préférable de barrer partiellement les passes $n^{\circ} 3$ et 4 (ce qui était devenu possible sur le chantier).

En ce qui concerne la premiere question, le croquis $\mathrm{E}_{m}$ représente les fonds obtenus sur le modèle après l'écoulement des débits correspondants aux débits réels observés entre le 29 octobre et le 26 novembre. On voit que le croquis $\mathrm{E}_{m}$ est assez comparable au croquis $\mathrm{E}$, ce qui confirme que les mouvements de fond sont reproduits convenablement par le modele.

Au sujet de la seconde question, les essais ont montré que la reconstitution du seuil dans la 

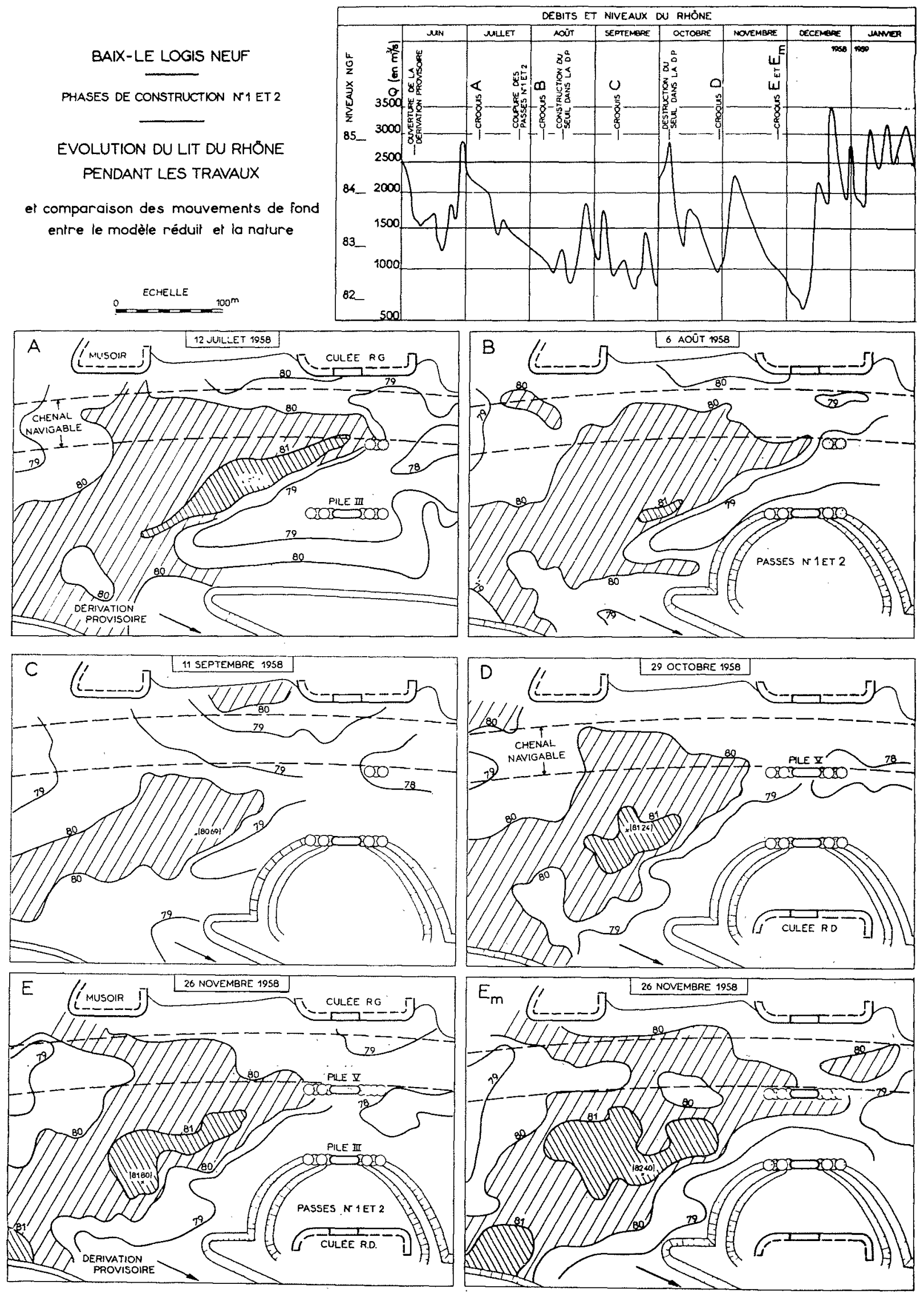

Fig. 13

Exemples de l'Évolution du lit du RHONe PENDANT la construction d'Un barkage.

Le dessin se rapporte à la construetion du barrage de Lotiol (aménagement de Baix-Le Logis Neuf). Il montre les modifications du fond du lit intervenues pendant une période de six mois environ (fin de la phase des travaux $n^{\circ} 1$ et début de la phase $n^{\circ}$ 2). Ce dessin permet également de comparer les mouvements de fond constatés sur le modèle réduit à ceux observés dans la nature. 
tent un accroissement de la hauteur de chute dans l'aménagement considéré et dans celui d'amont; lorsqu'ils sont exécutés dans la partie amont des retenues, ils favorisent également la navigation par une réduction des pentes et des vitesses $\mathrm{du}$ courant. Mais la réalisation de ces agrandissements du lit est fort onéreuse; on ne peut donc aller trop loin dans cette voie; de plus, il faut étudier avec soin leur répercussion éventuelle sur le cheminement du débit solide.

\subsubsection{Principales Études hydrauligues.}

En définitive, des études très complètes (techniques et économiques) sont nécessaires pour trouver le meilleur compromis entre les différents intérêts en cause : protection des riverains, développement industriel ou agricole de la vallée, augmentation de la production hydroélectrique et amélioration de la navigation, limitation du coût des aménagements et de leur exploitation (dragages éventuels d'entretien). Ces études doivent permettre de définir en conséquence: la nature des digues (submersibles ou insubmersibles), leur implantation exacte, la position et l'importance des agrandissements du lit mineur.

Les modèles réduits réalisés pour les études des prises d'eau et des barrages représentent généralement la partie aval des retenues, sur une longueur de 2 ou $3 \mathrm{~km}$. Les études d'implantations des digues et des agrandissements du lit mineur, pour ces tronçons de retenues, peuvent donc être effectuées avec l'aide des modèles en question (par de nombreuses comparaisons de lignes d'eau mesurées sur ces modèles).

Les études sont ensuite poursuivies vers l'amont, en déterminant les lignes d'eau par le calcul. Mais les calculs d'écoulement en régime varié sont laborieux : pour obtenir une précision suffisante, il faut prendre en compte des profils du Rhône assez nombreux; les calculs doivent être effectués pour une dizaine de débits du fleuve échelonnés de l'étiage à la crue millénaire, et plusieurs cas de fonctionnement doivent être considérés (retenue normale, chasses, crues, etc.). Préalablement, il convient de déterminer les coefficients de rugosité à utiliser, en se basant sur des lignes d'eau observées dans l'état naturel et en apportant les ajustements nécessaires. Enfin, des comparaisons doivent être effectuées pour différentes implantations de digues et pour des agrandissements du lit mineur plus ou moins importants.

$\mathrm{Au}$ total, ces études représentent un travail matériel assez considérable si on veut obtenir une précision suffisante. Nous avons pu mettre au point une méthode permettant de confier les calculs proprements dits à une calculatrice électronique. Le travail préliminaire de préparation reste très important, mais on peut obtenir ensuite quasi instantanément un grand nombre de résultats. C'est ce qui a été fait pour les retenues de Beauchastel et Pierre-Bénite.

Dans certains cas particulièrement délicats (régions submersibles habitées), nous sommes amenés à construire des modèles réduits complémentaires pour comparer les conditions de submersion dans l'état naturel et après réalisation de l'aménagement. Ces modèles permettent accessoirement de venir en aide aux calculateurs, car l'écoulement au droit des zones submersibles ne peut être défini avec une approximation suffisante par les seuls moyens mathématiques. Des modèles de ce genre ont été réalisés pour la chute de Baix-Le Logis Neuf (île de Printegarde, qui constitue un champ d'inondation de $500 \mathrm{ha}$ ) et celle de Beauchastel (îles de Blaud et du Chaffit).

Lorsque les travaux d'aménagement d'une retenue sont complètement définis, les calculs de lignes d'eau et de remous indiqués ci-dessus sont complétés par la détermination des pentes et des vitesses moyennes du courant, ce qui permet d'étudier les conditions de transport des matériaux graveleux ou en suspension et de mettre au point les consignes d'exploitation dont il est fait mention par ailleurs. Le report des chiffres obtenus sur des diagrammes de vitesses ou de pentes classées (fig. 14 et 15) donne aussi des indications précieuses sur les conditions qui sont réservées à la navigation dans les retenues en période de crues.

Le réaménagement nécessaire du confluent des principaux affluents qui débouchent dans les retenues (10) donne également lieu à des études diverses et notamment sur modèle réduit. A titre d'exemple, citons le cas de l'Eyrieux, torrent cévenol aux crues puissantes, dont le cours inférieur a dû être réaménagé sur un kilomètre au débouché de la retenue de Baix-Le Logis Neuf. II en a été de même pour la Drôme, dont le cours inférieur a dû être endigué sur, une longueur double et muni d'un important piège à graviers. Nous avons rendu compte ailleurs [21 et 23] de cette étude qui a permis d'utiles confrontations entre le charriage réel et celui observé sur modèle réduit. Nous étudions actuellement, dans des conditions analogues, les confluents de la Saône (retenue de Pierre-Bénite), de la Durance et du Gardon (chute de Vallabrègues).

(10) Nous ne parlons pas ici des affluents qui débouchent dans le Rhône courtcircuité, ou qu'il faut se résoudre à capter dans la dérivation (Roubion à Montélimar, Isère à Bourg-lès-Valence). Les problèmes hydrauliques posés (évacuation des crues, cheminement du débit solide, influence des courants traversiers sur la navigation) sont également délicats et en grande partie du ressort du modèle réduit. 
Fis. 14

Vitesses Molennes dans Les Retenues de Donzere et dr Montriman (en fonction du débit $Q$ du fleuve).

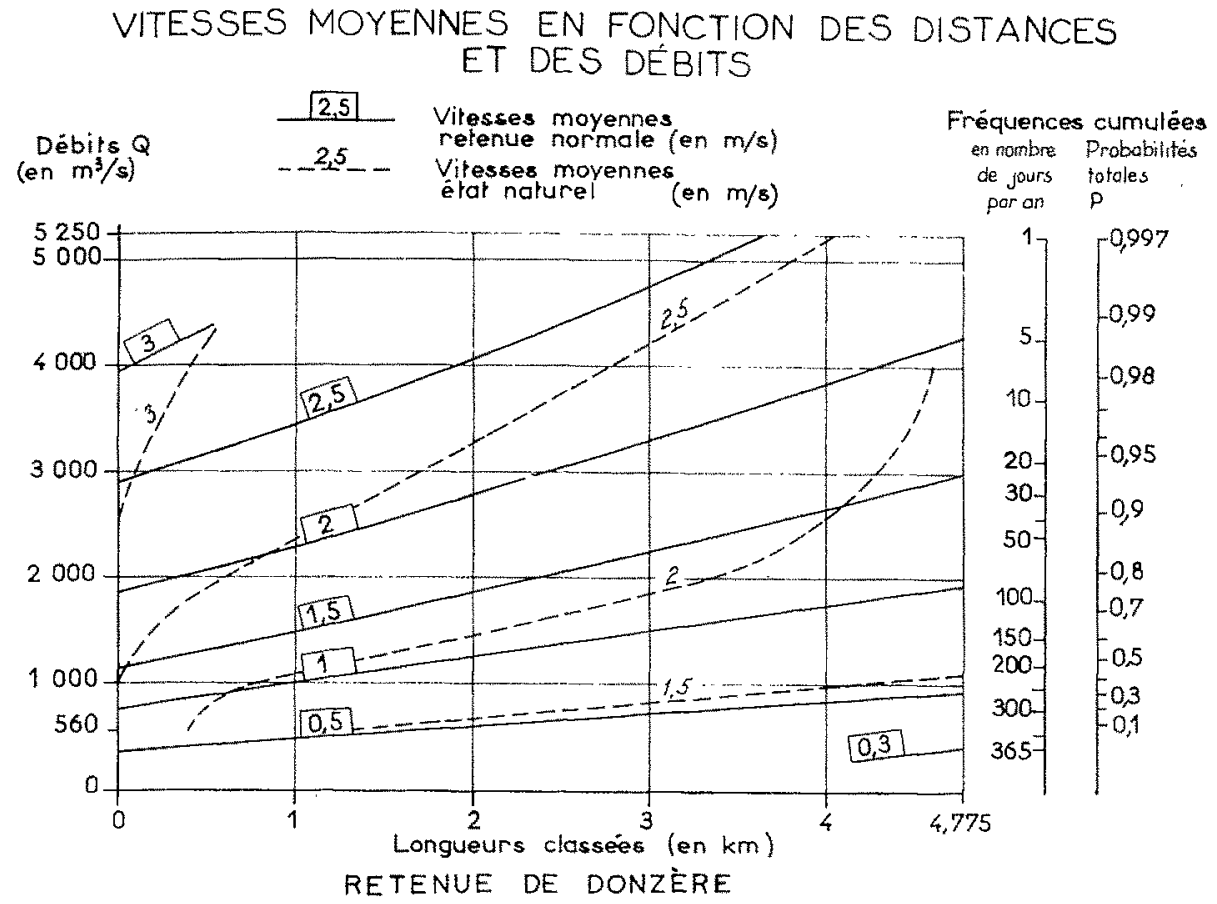

Dëbits $Q$

(en $\mathrm{m}^{3} / \mathrm{s}$ )

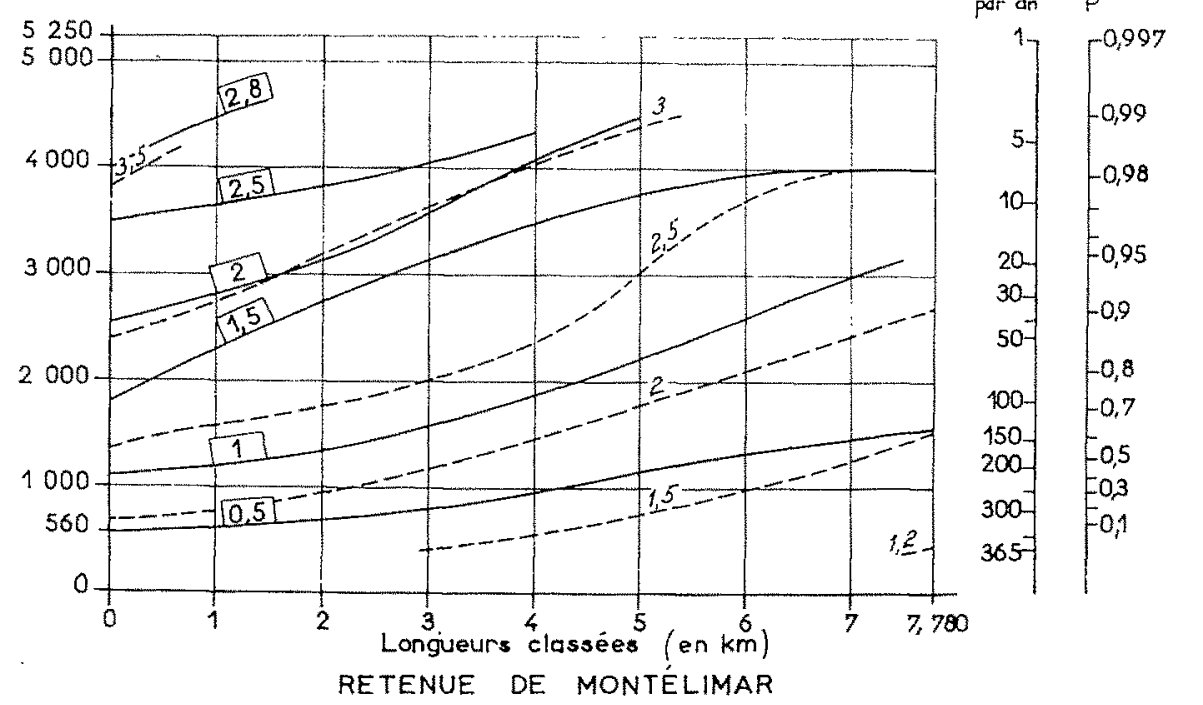

Les vitesses sont classées d'après les longueurs des tronçons où tlles règnent. Ces courbes permettent la comparaison entre l'état naturel cavant aménagement) et le régime normal d'exploitation.

Les courbes permettent egaleinent de comparer le cas d'une retenue basse (chute de Donzère-Mondragon) à celui d'uno retenue haute (chute de Montelimar).

L'examen comparatif de ces deux graphiques est riche d'enseignements :

a) Dépót des materriaux en suspension :

Dans l'etat naturcl, la vitesse moyenne ne descend jamais audessous du seuil de dépôt des matérianx en suspension $\left(V_{x}=1 \mathrm{~m} / \mathrm{s}\right)$. A Donzère (rete. nue basse), on constate que $100 \mathrm{j} / \mathrm{an}$ an moins les vitesses sont superieures à ce chiffre sur loute la longueur de la retenue, si bien que les rares dépots iventuels de materiaux fins sont régulièrement mobilisés et évacués par le barrage. A Montelimar au contraire (retenue haute), les matériaux fins peuvent se déposer pendant plus de $355 \mathrm{j} / \mathrm{an}$, au moins dans une partic de la retenue. Seuls le passage des grandes erues ou lexécution de chasses veuvent les mobiliser.

b) Charriage :

Dans l'ctat maturel du fleuve, Ja vitesse moyenne dépasse le seuil de charriage $\left(V_{o}=2 \mathrm{~m} / \mathrm{s}\right)$ sur la moitie de la longueus totale pendant une durée d'environ 150 jours par an. Apres aménagement, sauf manouvre spéciale de chasse, ces vilesses ne sont respectivement depassées que $18 \mathrm{j} / a n$ à Donzère et 4 j/an à Montélimar.

c) Novigation dans les retenues: Dans l'état maturel, les vitesses s'échelonnent sur l'ensemble du parcours du fleuve entre 2 et $4 \mathrm{~m} / \mathrm{s}$ pour la gamme des débits navigables (de 500 i $4000 \mathrm{~m}^{3} / \mathrm{s}$ ). Après aménagement, la retenue de Montelimar earacténise assez

bien la situation moyenne des retenues. Le graphique montre que la vitesse moyenne du courant y est diminuée d'environ $1 \mathrm{~m} / \mathrm{s}$ par rapport à la situation naturelle. La puissance de pointe nécessaire aux bateaux pour franclir les forts courants peut done être fortement diminuée. Mais le bénéfice est en réalité beaucoup plus considérable : les retenues ne règnent que sur moins de la moitié du parcours total alors que, dans les canaux, la vitesse est comprise entre 0,5 et $1,5 \mathrm{~m} / \mathrm{s}$, ce qui correspond à une diminution comprise entre 1,5 et $2,5 \mathrm{~m} / \mathrm{s}$ par rapport a la vitesse naturelle. A Donzère, l'amélioration par rapport à la situation antérieure (zone de rapides) est considérable, mais les vitesses résiduelles sont un peu plus élevées que précédemment, tant que certains travaux complémentaires envisagés ne seront pas réalisés. 


\section{PENTES CLASSEES SELON LA DISTANCE (en $\mathrm{cm} / \mathrm{km}$ )}
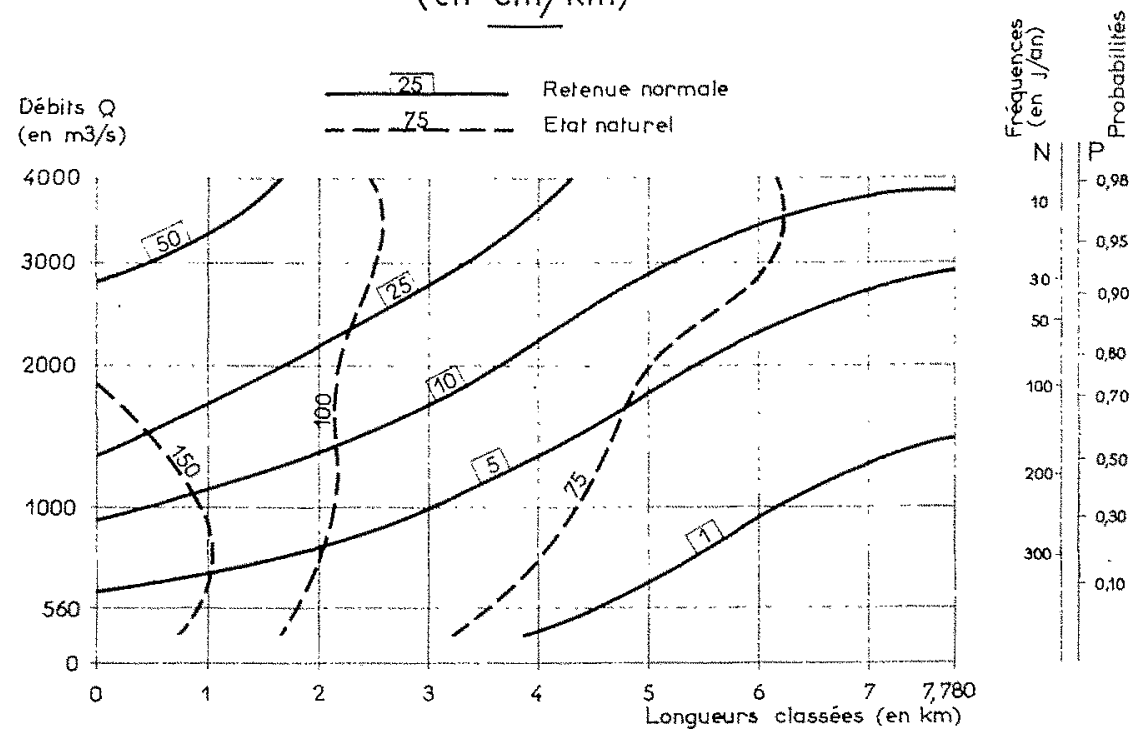

RETENUE DE MONTELIMAR

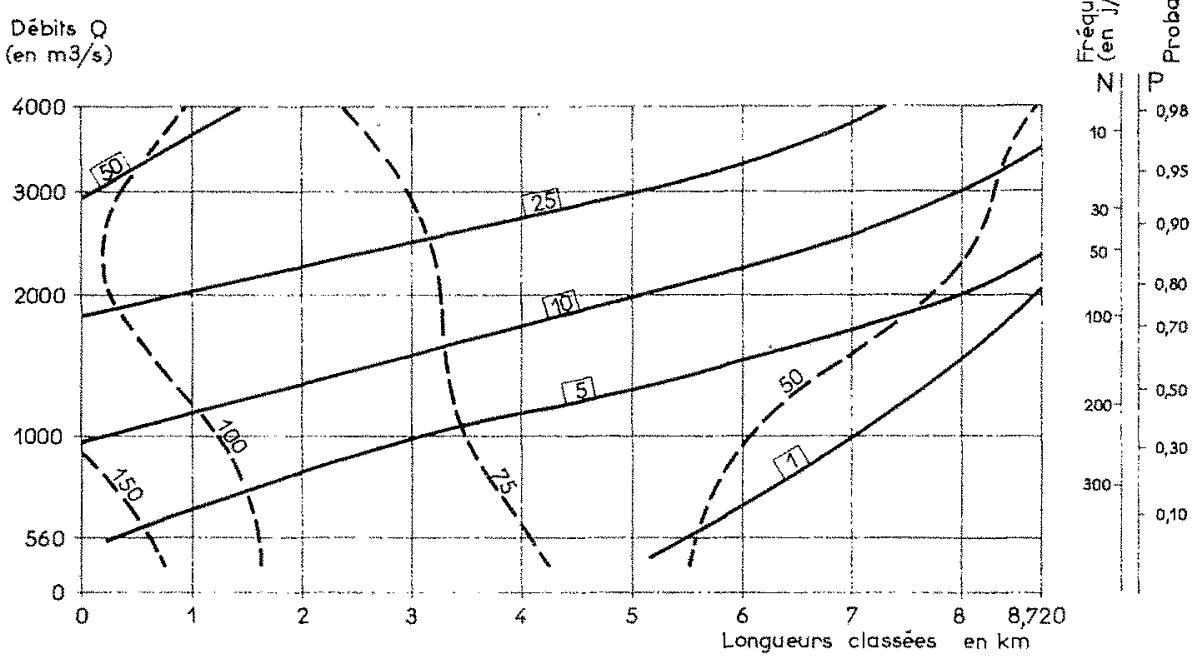

Fig. 15

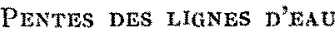
DANS LES RETENUES DE MonTÉllmar

ET BATX-Le Logis NeUf.

Les graphiques des pentes ont été établis de la même manière que les graphiques des vitesses moyennes (fig. 14). Ils permettent d'apprécier la réduction cousidérable des pentes dans les retenues par rapport à l'état naturel. Les deux aménagements considérés étant à retenue haute, les courbes correspondantes sont très comparables.

RETENUE DE BAIX-LE LOGIS NEUF

\section{2. - LA PRISE D'EAU}

L'implantation de la prise d'eau d'un aménagement mixte (énergie et navigation), sur un fleuve au débit solide relativement abondant, est une opération toujours délicate. En particulier, la prise doit satisfaire aux impératifs suivants : - prélever le débit liquide en évitant le dépót des matériaux en suspension, ainsi que l'introduction des matériaux de charriage dans la prise ou dans le canal;

- obtenir une répartition très régulière des vitesses à l'entrée évitant les courants traversiers gênants pour la navigation et les pertes de chute onéreuses pour la production énergétique.

Les conditions de fonetionnement d'une prise dépendent d'un grand nombre de paramètres que divers auteurs ont essayé d'analyser séparément [1 et 2,11 et 12]. Les uns sont liés aux dimensions et aux caractéristiques géométriques de la prise, les autres aux conditions de débit et de niveau du plan d'eau. Tous ces facteurs concourent en réalité à obtenir une bonne répartition des vitesses du courant dans la prise, qui semble être l'objectif essentiel à atteindre. 
Tableau III. - Caractéristiques géométriques des prises d'eau.

Cie tableau rassemble, pour les cinq aménagements du Bas-Rhône, les valeurs des paramètres essentiels qui caractérisent une prise d'eau.

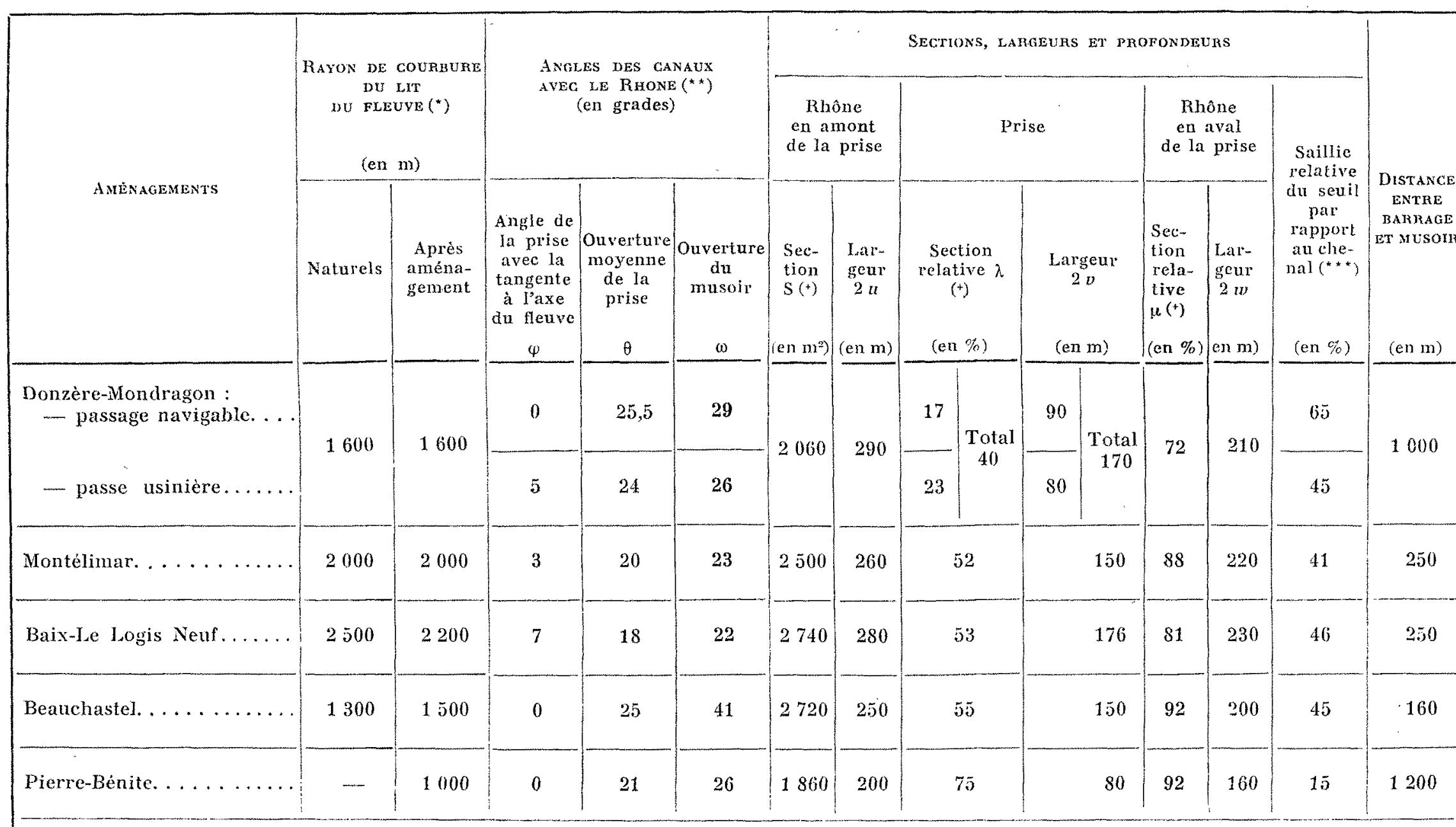

(") Rayon mesuré à $\pm 200 \mathrm{~m}$ près.

(*) Angles mesurés à \pm 1 à 2 gr près. Si on mesure en grades l'angle que fait l'axe du canal avec la tangente au chenal le plus profond du fleuve, on trouve $\mathrm{DM}=20, \mathrm{M}_{0}=18, \mathrm{~B} . \mathrm{L} . \mathrm{N} .=15, \mathrm{~B}_{\mathrm{e}}=18$, P.B. $=13$. D'autre part, à Bourg-les-Valence, les angles du canal avec le Rhône prennent les valeurs respectives suivantes : $\varphi=0 ; \theta=37 \mathrm{gr}$ et $\omega=44 \mathrm{gr}$.

$\left({ }^{* *}\right)$ Cette saillie relative est comptée par rapport à la profondeur du chenal sous la cote normale d'exploitation.

$(+)$ Les sections mouillées correspondant à la cote normale d'exploitation de l'aménagement (pour $Q=q_{e}$ ). Les sections relatives $\lambda$ et $\mu$ sont le quotient de la section de la prise ou du Rhône aval par la section $S$ du Rhône amont. 
La façon dont interviennent les différents paramètres fait que chaque aménagement est pratiquement un cas d'espèce et qu'une étude détaillée sur modèle réduit s'impose dans tous les cas.

De plus, des conditions étrangères à l'hydraulique doivent aussi être prises en considération : présence d'agglomérations en bordure du fleuve, existence de terrasses alluviales, recherche d'un bon terrain de fondation pour le barrage, etc. Au total, afin de concilier les différentes conditions, on est parfois conduit à remodeler le lit du fleuve dans la région de la prise et du barrage.

\subsection{Caractéristiques géométriques de la prise.}

Les principaux facteurs qui interviennent dans la définition géométrique de la prise d'eau sont : l'importance de la courbure du fleuve au voisinage de cette prise, la valeur de l'angle que forme l'axe de la dérivation avec l'axe du fleuve, les seclions respectives de la prise et du fleuve, la nature et la profondeur du seuil, la distance entre prise et barrage (tabl. III).

D'une manière générale, pour éviter l'engravement d'une prisé d'eau ef obtenir un entonnement correct du débit dérivé, il faut que la prise soit implantée sur la rive extérieure d'une courbe naturelle du fleuve, le canal d'amenée étant situé tangentiellement à cette courbe (ou formant avec la tangente un angle faible dirigé vers l'extérieur de la courbe) : ainsi, les courants de fonds chargés du débit solide de charriage suivent la courbure en direction du barrage, et une mouille se maintient devant la prise, mouille que l'on peut d'ailleurs accentuer par un seuil artificiel. Mais les conditions les meilleures au point de vue du débit solide ne sont pas toujours les plus favorables pour une bonne dérivation du débit liquide.

Nous allons indiquer succinctement l'influence des différents paramètres en cause et examiner quels sont ceux qui ont été déterminants dans les différentes prises du Rhône.

\subsubsection{Courbure du Fleuve.}

D'une manière générale, une courbure importante du fleuve, qui crée une forte dissymétrie des vitesses dans le profil en travers, permet d'éviter l'introduction du débit solide dans la prise. Mais elle entraîne de fortes vitesses à l'extérieur de la courbure, notamment au voisinage du musoir.

Inversement, si la courbure du fleuve au voisinage de la prise d'eau est trop faible, le courant d'eau est mal guidé vers le canal. Les vitesses dans la prise sont inégalement réparties: les plus grandes vitesses règnent au voisinage du musoir d'entrée, ce qui tend à diriger les bateaux trop près de ce musoir; au surplus, si la vitesse maximale est trop grande, il risque de se pro- duire des affouillements au pied du musoir, ce qui oblige à constituer un ouvrage très solide avec des fondations profondes, voire à le com pléter par un seuil en béton construit transversalement dans le plafond de l'entrée.

Vers la berge du canal opposée au musoir, il peut au contraire se produire des décollements de la veine liquide avec des zones d'eau morte, et même des courants de retour, ce qui favorise le dépôt des limons transportés en suspension par le fleuve; ce phénomène est d'autant plus intense en période de crue que le barrage est partiellement ou complètement ouvert.

En définitive, le rayon de courbure optimal est compris dans un certain intervalle, qui dépend d'ailleurs des autres caractéristiques de la prise.

Lorsque la courbure naturelle du fleuve n'est pas la plus favorable, il est possible de la corriger en déplaçant légèrement le lit du fleuve ou en remodelant son profil en travers par des guideaux longitudinaux qui attirent les courants, on des épis transversaux qui les freinent.

\subsubsection{ANGLe du CANAL AVEC LE FLEUVE.}

Lorsque le canal de dérivation ainsi que le fleuve en amont et en aval de la prise sont constitués de troncons rectilignes, il est facile de définir les angles (comptés dans le sens trigonométrique) que font le tronçon amont, d'une part avec la prise $(\alpha)$, et d'autre part avec le troncon aval $(\beta)$.

Lorsqu'on se trouve dans une courbe du fleuve, l'examen de la figure 16 (voir les notations employées) montre qu'on est amené à définir trois angles: à l'axe du fleuve (qui correspond sensiblement à $\alpha$ ), w ouverture du musoir, et l'angle intermédiaire $\theta$ qui semble correspondre à l'ouverture moyenne de la prise $(\theta \approx \alpha-\beta)$.

Les angles 0 et $\omega$ ne sont pas indépendants. Ils sont liés notamment à l'angle de la prise $\varphi$ ainsi qu'à la courbure par les relations :

$$
\begin{aligned}
& \cos \theta=\frac{\mathrm{R} \cos \varphi}{\mathrm{R}+w} \\
& \cos \theta=\frac{\mathrm{R} \cos \varphi--v}{\mathrm{R}+w}=\cos \theta-\frac{v}{\mathrm{R}+w}
\end{aligned}
$$

On voit immédiatement que lorsque $R$ est grand par rapport à $v$ et $w$, $\theta$ et $\omega$ tendent l'un et l'autre vers $\bullet$. D'autre part, lorsque $\%$ tend vers zéro, $\theta$ et $\omega$ tendent respectivement vers :

$$
\operatorname{arc} \cos \left(\frac{\mathrm{R}}{\mathrm{R}+w}\right) \text { et } \operatorname{arc} \cos \left(\frac{\mathrm{R}-v}{\mathrm{R}+w}\right) \text {. }
$$

La valeur optimale de l'ouverture de la prise est donc en partie liée à l'importance de la courbure et à la largeur des trois tronçons confluant à la prise. La différence $\omega-\theta$ entre l'ouverture 


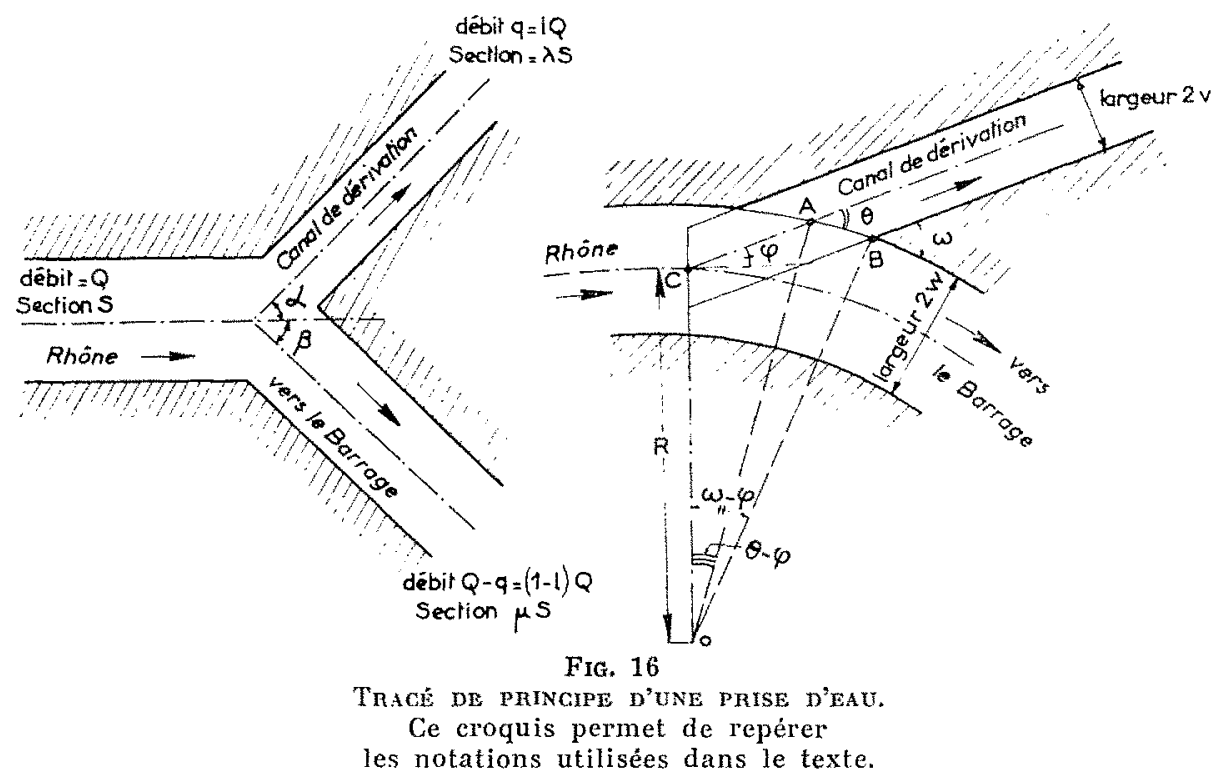

du musoir et celle de la prise est d'autant plus grande que la courbure est plus forte.

Nous avons vu que, pour éviter l'introduction des graviers dans la prise, il convient que l'angle - soit nul ou faiblement positif et que l'ouverture de la prise $\theta$ soit relativement grande. Cependant, si les angles $\theta$ ou $\omega$ sont trop ouverts, la vitesse du courant est trop grande au voisinage du musoir, et il se forme au contraire des zones d'eau morte sur la rive opposée de la prise. Inversement, si les angles sont trop faibles, l'élargissement de la section d'écoulement au droit de l'entrée règne sur une plus grande longueur; l'étendue de la zone de faible vitesse croît, et il en résulte, en période de charriage, des dépôts de gravier devant la prise.

\subsubsection{LE SEUIL.}

La section de la prise doit être suffisante pour que les vitesses ne provoquent pas d'érosion locale et ne facilitent pas l'introduction des matériaux charriés pour les forts débits dérivés et les faibles hauteurs de retenue. Trop grande, elle provoquerait par contre un ralentissement du courant, générateur de dépôt des matières en suspension dans la prise, ou des matériaux de charriage dans le lit du fleuve.

D'autre part, il n'y a jamais intérêt à prévoir une prise trop profonde: une certaine revanche doit être prévue par rapport au chenal du fleuve. Celle-ci doit être forte (Donzère) ou peut être modeste (Pierre-Bénite) selon le risque plus ou moins grand d'introduction des graviers dans la prise d'eau que font courir les autres caractéristiques de l'entrée (notamment l'importance du charriage naturel et des vitesses du courant sur le seuil).

Si la courbure du lit est très marquée, les mouilles qui se forment naturellement sur la rive concave du fleuve s'opposent à l'introduction des matériaux; mais il faut protéger la berge contre les ćrosions, par exemple grâce à des enrochements (Beauchastel) ou des ouvrages en béton (Donzère).

Si la courbure naturelle est pen marquée, ou la distance entre prise et barrage trop grande, on peut être amené à remodeler les fonds et à favoriser l'action des chasses par l'exécution d'un seuil sur une partie de la largeur de la prise. Ce seuil, qui se raccorde au musoir, peut d'ailleurs faire un certain angle avec la berge (Montélimar et Baix-Le Logis Neuf).

\subsubsection{IMPLANTATIONS RESPECTIVS DU BARRAGE E'T DE LA PRISE.}

Alimentation du barrage. - Afin que le débit soit bien réparti sur toute la largeur du lit et que le débouché de l'ouvrage soit pleinement utilisé, il est préférable d'implanter le barrago en un point d'inflexion du lit du fleuve, ou plus précisément au point d'inflexion «hydraulique » où les vitesses sont réparties de manière symétrique dans le profil en travers du lit; plus la courbure est forte, plus ce point est silué en aval du point d'inflexion « géométrique».

Ainsi, quand la courbure du lit est très marquée, il est généralement préférable de placer le barrage assez loin de la prise d'cau (celle-ci est, en général, implantée au sommet de la courbe). C'est le cas des aménagements de Donzère (11)

(11) Bien qu'éloigné de lá prise, le barrage de Donzère a dû être placé, pour diverses raisons, au point d'inflexion gémétrique et non hydraulique : la dissymélyie des vitesses qui résulte de cette situation a contribuc a gener le passage des baleaux pendant certaines phases de la construction de l'ouvage. 
et de Pierre-Bénite, et il en sera vraisemblablement de même pour celui de Bourg-lès-Valence.

Inversement, lorsque la courbure du lit est peu marquée, la dissymétrie du courant est faible et le barrage peut, sans inconvénient majeur, être placé notablement en amont de sa position optimale théorique. C'est ce qui explique que la distance entre la prise et le barrage est beaucoup plus faible à Montélimar et à Baix-Le Logis Neuf (fig. 17) que dans les aménagements précités.

Interactions du barrage et de la prise. - Dans la réduction de la distance entre prise et barrage, on est cependant limité par une autre considération. En effet, si le barrage est trop près de la prise (distance inférieure à la largeur du lit, soit 150 à $200 \mathrm{~m}$ sur le Rhône), il se forme, lorsque le barrage ne débite pas, des rouleaux d'axe vertical dans la zone comprise entre les deux ouvrages. Ces rouleaux perturbent l'entrée de l'eau dans la prise : les vitesses y sont mal réparties et il naît des courants traversiers qui peuvent être gènants pour la navigation.

Par contre, si c'est l'usine qui est très proche de la prise (cas de Pierre-Bénite, où le canal d'amenée est très court), il y a intérêt à éloigner le barrage vers l'aval de manière à rendre aussi indépendantes que possible les alimentations de ces deux ouvrages.

Efficacité des chasses. - Enfin, il convient de tenir compte d'une autre condition, concernant le débit solide. En effet, si le barrage est trop éloigné de la prise (quatre à cinq fois la largeur du lit), les chasses n'ont qu'un effet médiocre sur les dépôts qui ont pu se former au droit et en amont de la prise. Pour éviter la formation de ces dépôts, il faut abaisser le niveau de la retenue, même pour les crues moyennes du fleuve, et réduire beaucoup le débit dérivé, ce qui diminue la production de l'usine. Lorsque le niveau normal de la retenue est élevé, la considération du débit solide est en général prépondérante, et il y a grand intérêt à rapprocher le barrage de la prise, en dépit des inconvénients qui peuvent en résulter pour la répartition du débit au barrage si la courbure du lit est notable (cas de Beauchastel).

Conclusions. - Au total, le choix de la distance entre prise et barrage résulte d'un compromis entre le désir d'obtenir la meilleure répartition du débit liquide au barrage et celui de favoriser le transit du débit solide dans la partie aval de la retenue. Le premier incite à

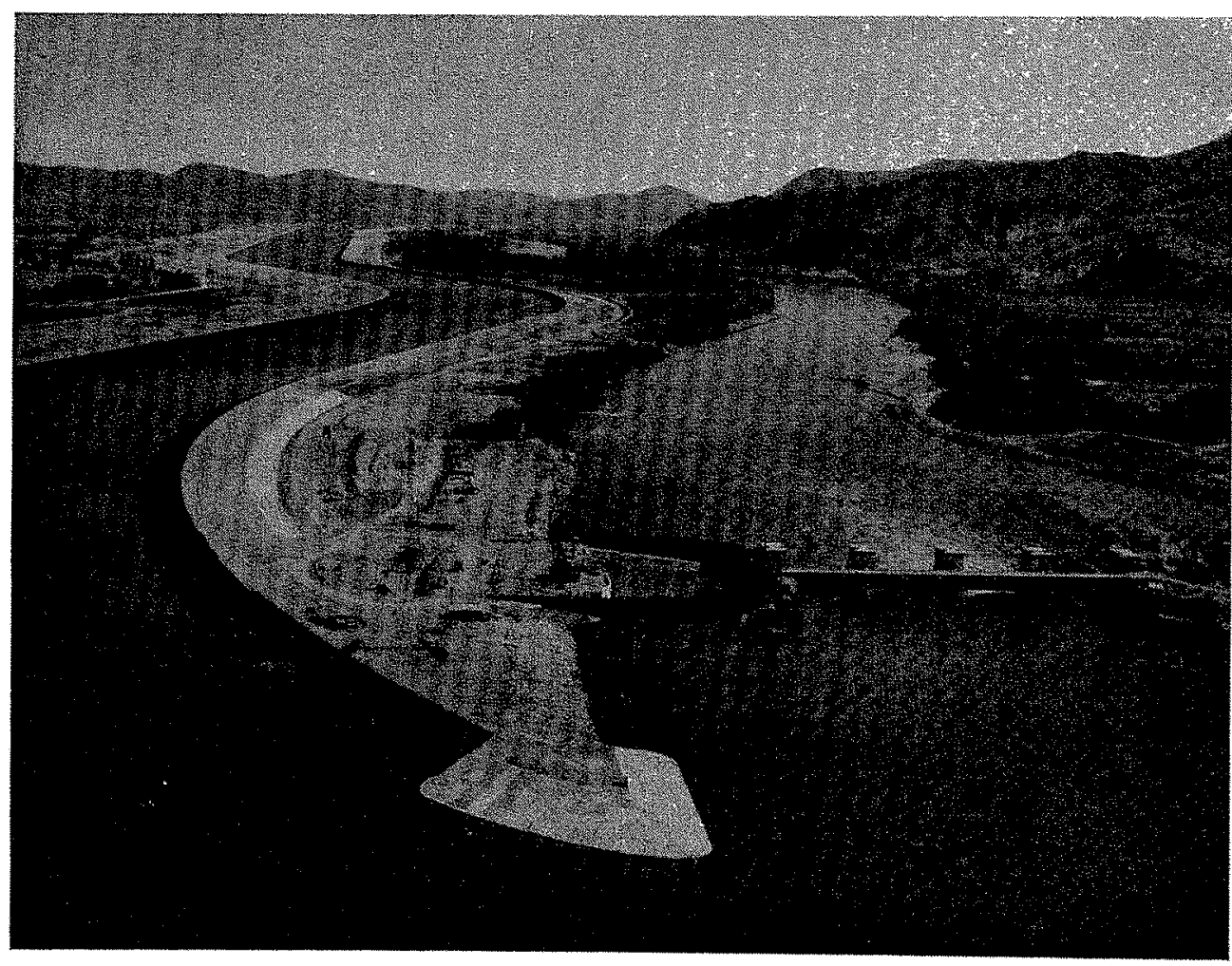

FIG. 17

(Photo Cellard,

UN Exemple de prise D'eav (aménagement de Baix-Le Logis Neuf).

Vue aérienne du canal et du barrage (au cours de sa dernière phase de construction) an premier plan : le musoix d'entrée. La retenue n'est pas encore portée à son niveau définitif. 
Tableau IV. - Prises d'eau oes aménagements de Bas-Rhone.

Ce tableau rassemble, pour les cinq amenagemcnis considérés et pour quelques fr'équences caractéristiques du déỏit $\mathrm{Q}$ du fleuve, les valeurs des débits, niveaux st vitesses moyennes du courant au droit des prises d'eau.

\begin{tabular}{|c|c|c|c|c|c|c|c|c|c|c|c|c|c|c|}
\hline & \multicolumn{3}{|c|}{$\begin{array}{l}\text { DÉB IT D'ÉQGIPEMENT } \\
\text { DE L'USINE }\end{array}$} & \multicolumn{2}{|c|}{$\begin{array}{c}\text { ETIAGE } \\
(355 j / a n)\end{array}$} & \multicolumn{2}{|c|}{$\begin{array}{l}\text { CRUE DḰPASSËE } \\
(10 \mathrm{j} / \mathrm{an})\end{array}$} & \multicolumn{2}{|c|}{$\begin{array}{l}\text { GRUE DÉPASSEE } \\
1 \mathbf{j} / \mathrm{an})\end{array}$} & \multicolumn{2}{|c|}{$\begin{array}{l}\text { DÉBIT MINIMAX } \\
\text { OUVERTURE } \\
\text { TOTALE } \\
\text { DU BARRAGE }\end{array}$} & \multirow{2}{*}{$\begin{array}{c}\text { DÉNIVEL- } \\
\text { I.ATION } \\
\text { ENTRE LE } \\
\text { NIVEAU DE } \\
\text { LA CRUE } \\
\text { MLLLE- } \\
\text { NAIRE } \\
\text { M ET DE } \\
\text { L'ETAGE } \\
\text { E } \\
\\
\text { (en m) } \\
\end{array}$} & \multicolumn{2}{|c|}{$\begin{array}{c}\text { POSITION } \\
\text { DES NIVEAUX } z \\
\text { DE RETENLE } \\
\text { PAR MAPPORT } \\
\text { A M ET E : } \frac{z-\mathrm{E}}{M-\mathrm{E}} \\
(\text { en } \%)\end{array}$} \\
\hline & $\begin{array}{c}q^{*} \\
\left(\mathrm{ean}^{\mathrm{n}}\right. \\
\left.\mathrm{m}^{3 / \mathrm{s}}\right)\end{array}$ & $\begin{array}{c}\text { Frú- } \\
\text { Quence } \\
\text { (en j/an) }\end{array}$ & $\begin{array}{c}\text { VITESSR } \\
\text { MOYENNE } \\
\left({ }^{*}\right) \\
(\mathrm{en} \mathrm{m} / \mathrm{s})\end{array}$ & $\frac{q_{0}}{Q}$ & $\begin{array}{l}\text { VITESSE } \\
\text { MOYENNE } \\
\left({ }^{*}\right) \\
(\mathrm{en} \mathrm{m} / \mathrm{s})\end{array}$ & $\frac{q 0}{Q}$ & $\begin{array}{c}\text { VITESSE } \\
\text { MOYENNE } \\
\left({ }^{*}\right) \\
\text { (en m/s) }\end{array}$ & $\frac{q_{e}}{Q}$ & $\begin{array}{c}\text { VTrESSE } \\
\text { MOYENNE } \\
\left({ }^{*}\right) \\
(\mathrm{en} \mathrm{m} / \mathrm{s})\end{array}$ & $\begin{array}{c}\mathrm{Q} m \\
(\mathrm{en} \\
\left.\mathrm{m}^{3} / \mathrm{s}\right)\end{array}$ & $\begin{array}{c}\text { Fris- } \\
\text { QUENCE } \\
\text { (cn j/an) }\end{array}$ & & $\begin{array}{c}\text { Chasse } \\
\text { Cote } \\
\text { minimale } \\
z=\mathrm{H}_{0}\end{array}$ & $\begin{array}{c}\text { Retenue } \\
\text { normale } \\
\text { Cote } \\
\text { maximale } \\
z=H_{n}\end{array}$ \\
\hline $\begin{array}{l}\text { Donzère-Mondragon : } \\
\text { - passe navigable. } . \\
\text { - passe usinière. . . } \\
\text { - total. . . . . . . . . . }\end{array}$ & $\begin{array}{r}600 \\
1100 \\
1700\end{array}$ & 113 & $\begin{array}{l}1,66 \\
2,30\end{array}$ & 3,33 & $\begin{array}{l}0,50 \\
0,69\end{array}$ & 0,46 & $\begin{array}{l}1,44 \\
2,04\end{array}$ & 0,32 & $\begin{array}{l}1,44 \\
2,04\end{array}$ & 4500 & 3 & 7,90 & 50,7 & 62,7 \\
\hline Montélïmar. . . . . . . & 1920 & 89 & 1,47 & 3.8 & 0,39 & $0, \overline{2} 2$ & 1,47 & 0,37 & 1,64 & 6000 & 0,3 & 7,78 & 54,7 & 119 \\
\hline Baix-Le Logis Neuf..... & 2100 & 72 & 1,45 & 4.12 & 0,36 & 0,57 & $1,7 \overline{3}$ & 0.40 & 1,96 & 5500 & 0,6 & 7,55 & 49,8 & 120 \\
\hline Beauchastel. . . . . . . . & 2100 & 60 & 1,41 & 4,47 & 0,31 & 0,61 & $1, \overline{3} 2$ & 0,42 & 1,91 & 5400 & 0,5 & 8,79 & 52,4 & 125 \\
\hline Pierre-Bénite. . . . & 1400 & 78 & 1,00 & 4,38 & 0,23 & 0,32 & 1,00 & 0,36 & 1,35 & 3500 & 2,2 & 8,26 & 48,5 & 69,8 \\
\hline
\end{tabular}

(*) Les vitesses moyennes au droit de la prise correspondent pour chaque chute aux niveaux normaux d'exploitation. 
éloigner le barrage de la prise, tandis que le second incite à le rapprocher. De plus, la distance doit être suffisante pour éviter les interactions gênantes entre l'alimentation du barrage et celle de l'entrée du canal.

\subsection{Conditions générales de niveau et de débit.}

Comme nous l'avons déjà relevé, la répartition des vitesses au droit de la prise dépend non seulement de la forme des ouvrages, mais aussi des conditions de débit et de niveau qui $y$ règnent (tabl. IV).

Au droit de la prise, le débit $Q$ du fleuve se partage en un débit dérivé $q$ (dont le maximum $q_{e}$ correspond en général au débit d'équipement de l'usine) et en un débit non dérivé $Q-q$ (qui ne doit pas descendre en dessous du débit minimal réservé $r$ ). Tant que $Q \leqslant q_{c}+r$, la satisfaction des besoins énergétiques de l'usine conduirait à dériver le débit maximal en laissant le barrage fermé et en maintenant la retenue au niveau maximal $\mathbf{H}_{m}$ compatible avec les installations riveraines et la cote de submersion des endiguements.

Lorsque $Q$ augmente, le barrage serait ouvert progressivement pour laisser passer le débit $Q-q_{\epsilon}$, la retenue étant maintenue à un niveau maximal qui peut d'ailleurs décroître quand $\mathrm{Q}$ augmente pour que le remous créé à l'amont ne provoque pas de submersion (12).

Au-delà d'un débit maximal $Q_{m}$, il est nécessaire en toute hypothèse d'ouvrir complètement le barrage pour ne pas créer de surélévation excessive dans la retenue. Il faut noter que, dans la partie aval de celle-ci, le niveau dépend non seulement du niveau $\mathrm{H}$ au droit de la prise et du débit $Q$ du fleuve, mais aussi du débit dérivé q. Celui-ci provoque en effet au droit de la prise un certain remous d'abaissement qui s'atténue rapidement vers l'amont.

Mais les nécessités d'exploitation doivent tenir compte d'autres considérations liées à l'évacuation du débit solide du fleuve. Il résulte de nos études antérieures $[22 ; 25]$ que le problème du charriage peut être schématisé comme suit sur le Bas-Rhône : lorsque la vitesse moyenne $\mathrm{V}$ dans un profil en travers donné dépasse un

(12) A Donzère et Pierre-Bénite (dont les retenues sont bordées par des digues submersibles), et méme à Montélimar (digue insubmersible), cest le nivean géméral de la retentue qui doit être abaissé en période de crues. A Baix, Beauchastel et Bourg-lès-Valence, les digues insubmersibles situees en aval font place a des digues submersibles dans la partie amont de la retenue; pour éviter de perdre de la chute en basses eaux, on ste. amené à faire pivoter les lignes d'eau au droit des points critiques de submersion pour la gamme des débits de crue.

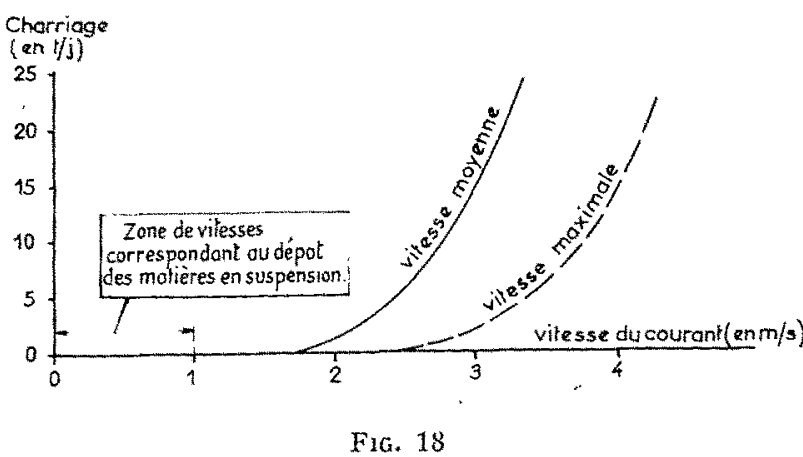

IMPORTANCE DU GHATRIAGE

EN FONCTION DE LA VITESSE DU COURANT (région de Donzère).

lic vitesse, moyenue ou maximale, est celle qui a une chance sur deux d'être dépassée dans un profil en travers quelconque du fleuve arant aménagement.

certain seuil de vitesse $V_{0}$ (la vitesse maximale dans le profil atteignant suivant le cas de $1,3 \mathrm{~V}$ à $1,5 \mathrm{~V}$ ), on observe un charriage généralisé des matériaux grossiers du fond du lit. La vitesse $V_{t}$, qui est de l'ordre de $2 \mathrm{~m} / \mathrm{s}$ sur le tiers central, correspond dans la situation naturelle du Rhône, à l'écoulement du débit critique $Q_{\text {. }}$ (voisin de $1500 \mathrm{~m}^{3} / \mathrm{s}$ ).

Pour des vitesses moyennes $\mathrm{V}$ supérieures à $V_{c}$, le charriage croît à peu près proportionnellement à $\left(\mathrm{V}^{2}-\mathrm{V}_{c}^{2}\right)^{3 / 2}$ ainsi que l'indique la figure 18. D'autre part, lorsque les vitesses sont très lentes et inférieures à une autre limite $V_{s}$ (de l'ordre de $1 \mathrm{~m} / \mathrm{s}$ ) les matériaux fins qui sont transportés en suspension par le fleuve ont tendance à se déposer.

La mise au point des consignes d'exploitation dont il est rendu compte ci-dessous montre qu'en période de crues, il n'est pas possible de dériver en permanence le débit maximal $q_{e}$ sous la cote de retenue maximale $\mathrm{H}_{m}$, sous peine d'engraver dangereusement la prise ou la retenue ou de favoriser l'envasement: en certaines circonstances, il est indispensable de diminuer temporairement le débit dérivé et la cote de la retenue.

Pour chaque chute, on est donc amené à définir un niveau normal d'exploitation $\left(\mathrm{H}_{t h} \leqslant \mathrm{H}_{m}\right)$ et même un niveau minimal de chasse $\left(\mathrm{H}_{0} \leqslant \mathrm{H}_{n}\right)$ correspondant aux différents dẻbits $Q$ du fleuve. Pour Baix-Le Logis Neuf, ces niveaux sont figurés sur la figure 27.

De manière à pouvoir juger de la situation respective des différents aménagements au point de vue de la fréquence des débits et des niveaux, nous avons reporté sur un même graphique la raleur des débits $q_{0}, \mathrm{Q}_{*}$ et $\mathrm{Q}_{m}$ pour les cinq aménagements considérés, et indiqué la fréquence avec laquelle le fleuve atteignait, dans 
son état naturel, les cotes $H_{n}$ et $H_{0}$ ci-dessus définies (fig. 19).

Dans tous les aménagements, on constate que le niveau minimal de chasse $H_{0}$ est voisin de celui dépassé par le fleuve libre un ou plusieurs mois par an. Les conditions de charriage naturel sont donc rétablies devant la prise, ce qui nécessite une forte diminution du débit dérivé et justifie le respect rigoureux des conditions géométriques (rappelées ci-dessus), propres à éviter l'engravement de la prise. Par contre, en retenue normale $H_{n}$, la situation à Donzère et Pierre-Bénite (où $\mathrm{H}_{n}$ est très bas), est bien différente de celles des autres retenues plus élevées. Il en résulte une assez grande diversité dans le détail des consignes d'exploitation propres à chaque aménagement.

\subsection{Etudes des différentes prises du Rhône.}

L'étude des prises a été menée avec un très grand soin sur des modèles réduits à fond mobile construits à grande échelle $(1 / 60$ ou $1 / 100)$. Le fleuve a été reproduit sur plusieurs kilomètres de longueur (à Baix-Le Logis Neuf : $5 \mathrm{~km}$, si bien que le modèle avait une longueur réelle totale de $85 \mathrm{~m}$ ). Les essais ont été effectués en reproduisant le passage d'un cycle de crue, soit à partir des fonds naturels, soit à partir de fonds supposés engravés (essais de sécurité). Dans diverses situations d'engravement et de débit de crue $Q$, on a fait varier indépendamment les deux paramètres $\mathrm{H}$ et $q$. La variété des dispositions auxquelles on a été conduit pour les prises témoigne de la diversité des conditions à respecter (fig. 20).

\subsubsection{RETENUES BASSES.}

Si la courbure est relativement forte à Donzère, le niveau très bas de la retenue entraîne des vitesses élevées au droit de la prise et des risques d'engravements pour des débits très fréquemment atteints. De plus, le Service de la Navigation a demandé que la passe navigable soit située dans l'exact prolongement du chenal amont, alors que l'emplacenent le plus favorable pour une prise mixte impliquait une légère courbure du chenal et une entrée située un peu plus en aval.

On a donc été conduit à réaliser une entrée double, dont les deux musoirs doivent être protégés contre l'érosion. La passe navigable, siluce dans la direction du courant, est dotée d'un seuil assez élevé limitant le débit qui l'emprunte. L'autre passe, située plus en aval, est chargée d'admeltre la plus grande partic du débit dérivé; le seuil qui l'équipe comporte un auvent de béton armé dont la forme a ćté étudiée pour favoriser le dégravement sous la cote de retenue normale et le débit d'équipement. Grâce à ces dispositions, on a pu accepter une assez grande distance entre prise et barrage (ce
Fig. 19

COMPARAISON DES FRÉQUENCES DES DÉBITS ET DES NIVEAUX.

Ce schéma permet de comparer, pour les cinq aménagements considérés, les fréquences des débits et des niveaux caractéristiques.

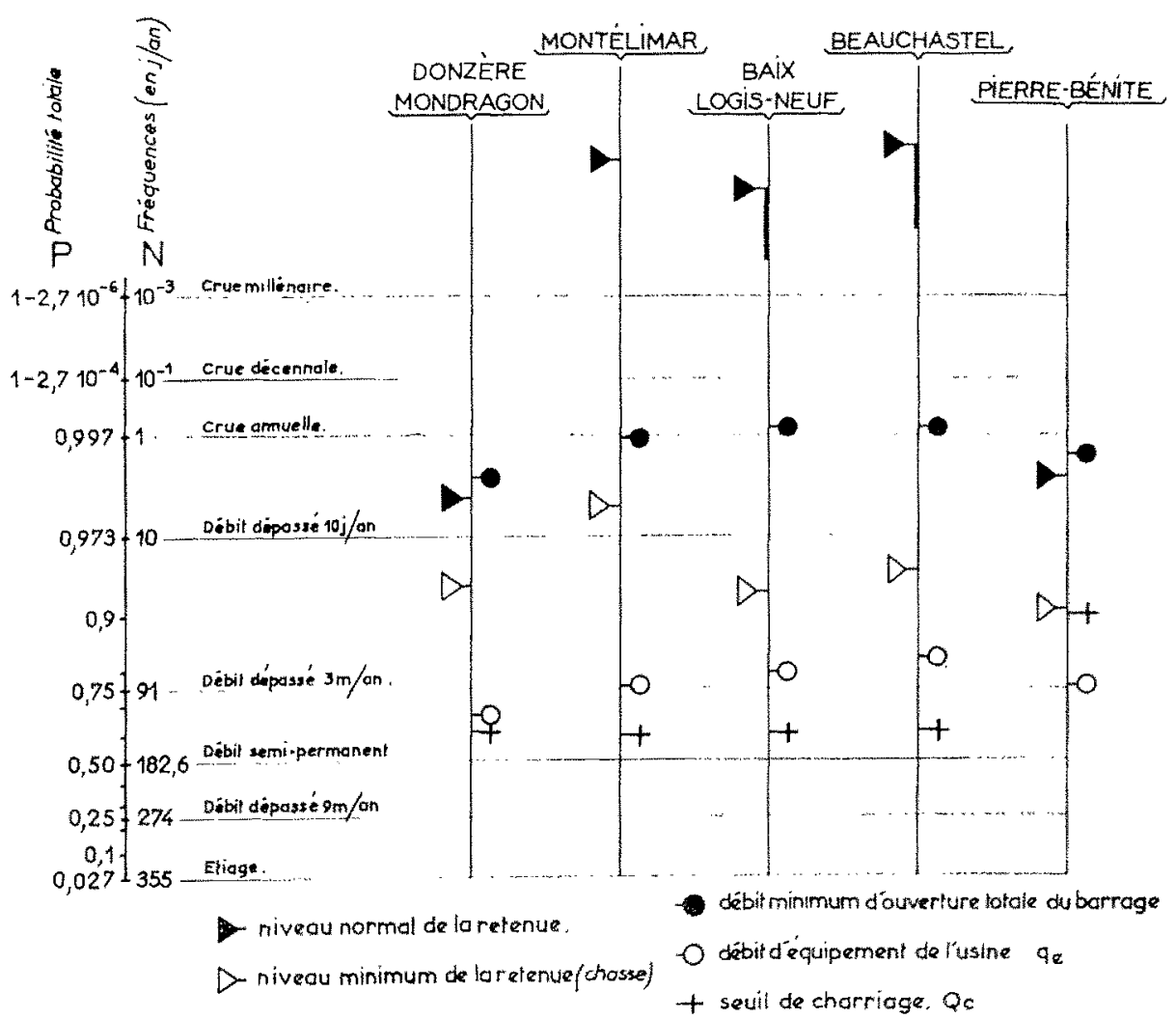




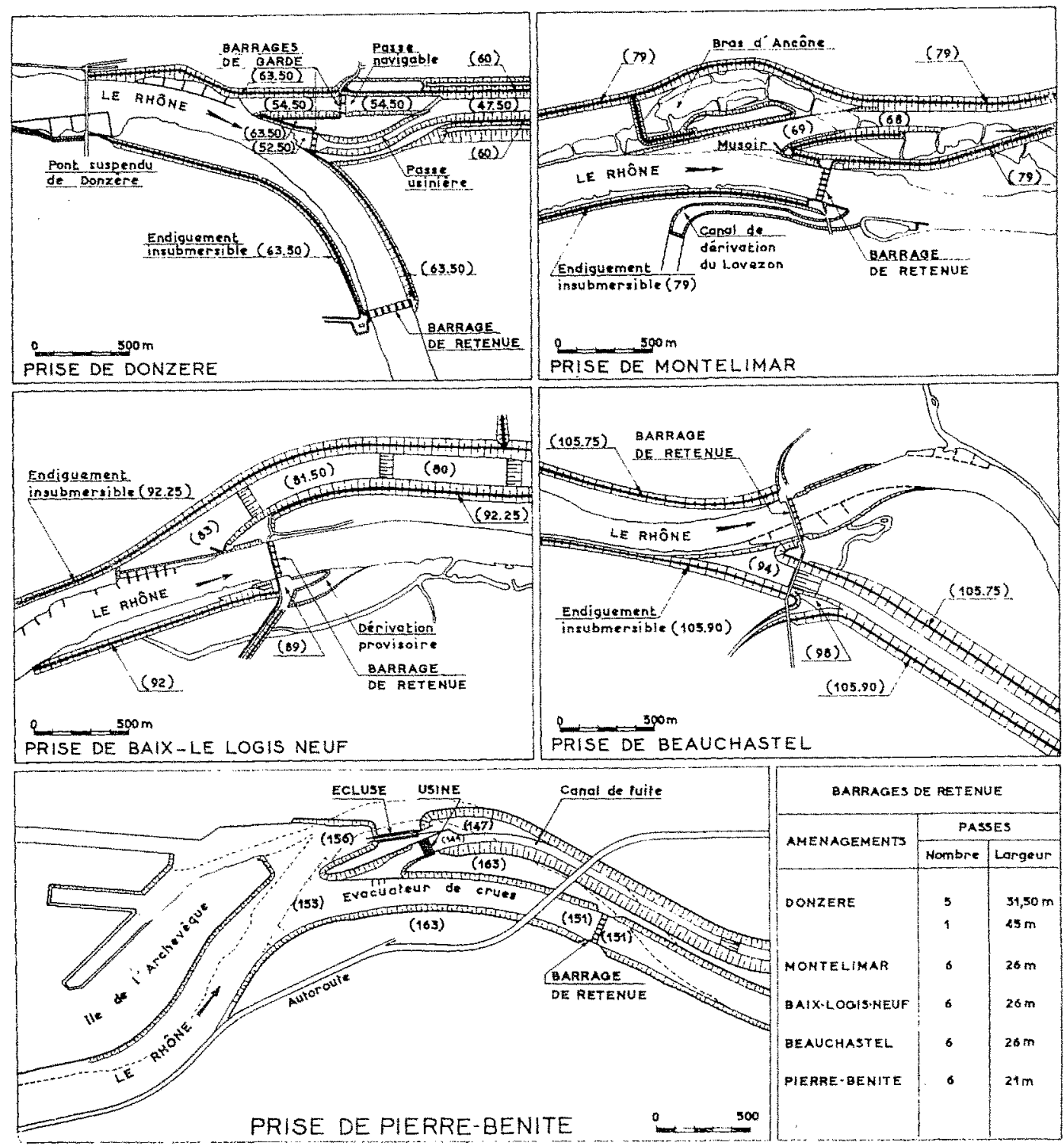

FIG. 20

PRISES D'EAU DES AMENAGEMENTS DU BAS-RHONE.

Plans schématiques des prises d'eau des trois aménagements existants (DonzèreMondragon, Montélimar et Baix-Le Logis Neut) et des deux aménagements en construction (Beauchastel et Pierre-Bénite).

qui est favorable à la bonne alimentation des passes de ce dernier) au prix, il faut le reconnaître, d'une moindre efficacité des chasses. En période de crue, comme nous le verrons plus loin, l'exploitation doit être menée avec une grande prudence, en raison des vitesses assez fortes qui règnent à l'entrée.

A Pierre-Bénite, la prise d'eau est entièrement artificielle. Compte tenu du débit plus modeste du fleuve (et par conséquent d'un débit d'équipement de l'usine plus réduit), ainsi que de la faible importance du charriage, les essais sur modèle ont conduit à une courbure beau- coup plus forte que dans les aménagements du tiers central.

Si les vitesses au voisinage de la prise restent assez modestes, une répartition régulière du courant a ćté particulièrement difficile à obtenir pour des raisons d'ailleurs très différentes du cas précédent : en amont des ouvrages, les vitesses les plus importantes sont situées en rive gauche par suite de la courbure, mais l'écoulement y est perturbé par la zone d'eau calme qui règne à l'entrée du port industriel et de l'écluse. Comme la prise alimente l'usine par l'intermédiaire d'un canal très court, toute dissymétrie des vitesses 
à l'entrée entraîne une répartition irrégulière de celles-ci devant les groupes de l'usine. Une implantation satisfaisante des ouvrages n'a pu être obtenue que par une étude très minutieuse des formes en plan et des talus des musoirs rive droite et rive gauche.

Bien qu'il ait fallu éloigner le barrage vers l'aval, il ne doit pas en résulter d'inconvénient pour le transit du débit solide et l'efficacité des chasses, en raison du faible charriage naturel du fleuve en aval du confluent avec la Saône, ainsi que du niveau bas de la retenue qui réduit les risques d'engravement.

\subsubsection{RETFNUES HAUTES.}

Dans la retenue haute de Beanchastel (fig. 20) un rayon de courbure voisin de celui de Donzère s'est avéré proche de l'optimum (pour les conditions de débit et de charriage régnant dans cette région). Nonobstant cette forte courbure du lit, le barrage a dû être implanté très près de la prise pour des motifs de fondation. La faible distance adoptée est évidemment très favorable à l'efficacité des chasses. Elle reste légè- rement supérieure alı minimum correspondant à la formation des rouleaux gênants pour la prise. En revanche, elle aurait pu ctre franchement insuffisante pour ce qui concerne la répartition du débit entre les différentes passes du barrage. Mais cet inconvénient a pu être corrigé dans une très large mesure, d'une part en décalant l'ouvrage par rapport au hit mineur du fleuve, d'autre part en le faisant piroler d'un certain angle par rapport à la normale à l'axc de ce lit. Grâce à ces dispositions, la mise au point de la prise d'eau n'a pas été très diflicile; le musoir est un simple massif en gravier protégé par des enrochements, el un seuil bétonné n'est pas nécessaire. Il en sera vraisemblablement de même à Bourg-les-Valence.

A l'inverse des cas précédents, la courbure était relativement faible à Montélimar. La mise au point de la prise d'eau n'a cependant pas présenté de grandes difficultés. Néanmoins, il a fallu fonder au rocher le musoir d'entréc en béton, et le compléter par un seuil également bétonné régnant sur une partie de la largeur de la prise. De plus, la présence d'une lòne

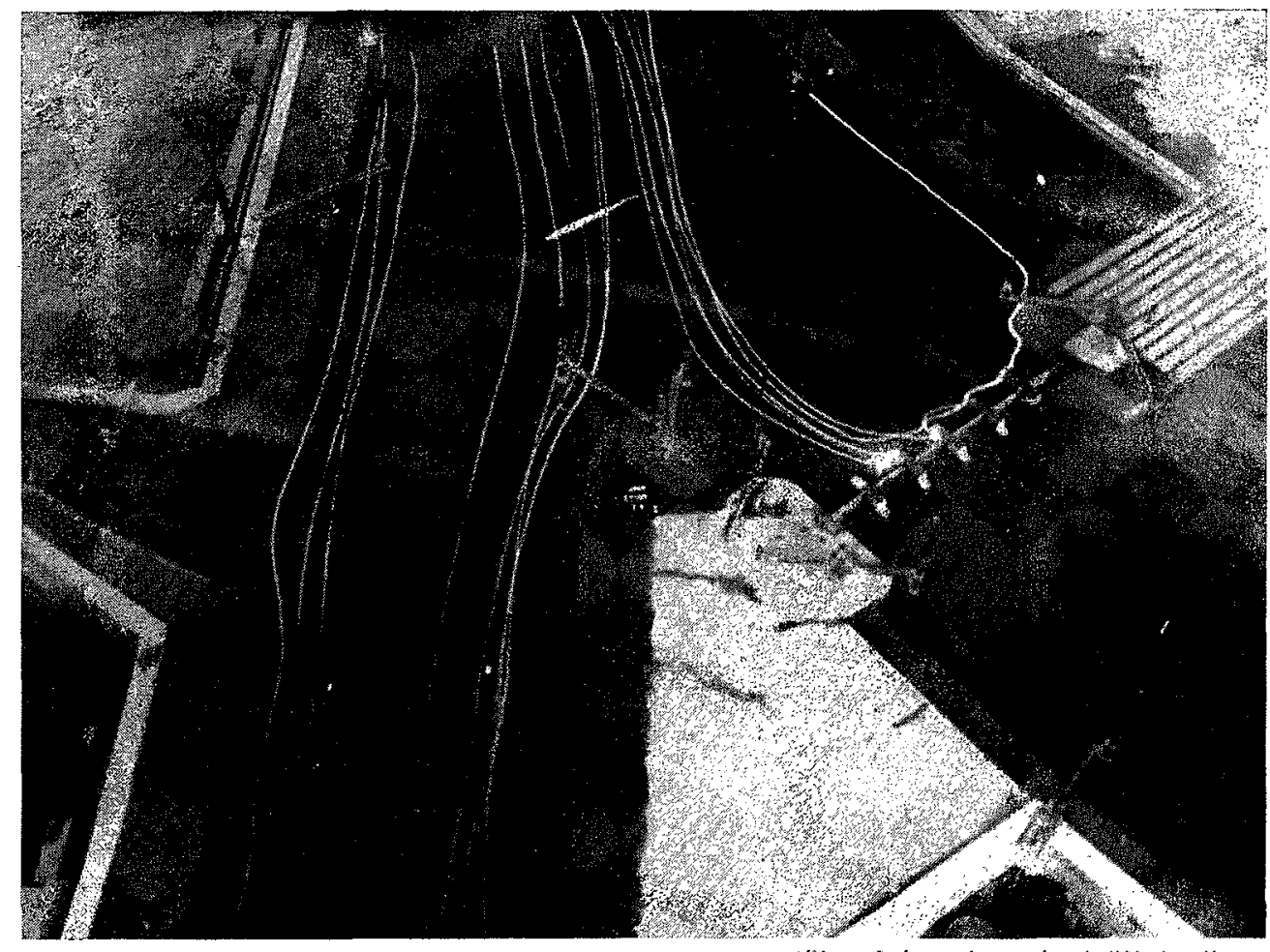

Fig. 21

(Photo Laboratoine nutional d'llydrauliuur,

ECOULEMENT DE L'EAU DANS DNE PHSY

(étude sur modèle réduit : aménagement de Beauchastel).

rue partielle du modèle réduit. Visualisation des lignes de courant dans l'entrée du canal d’amenée; le barrage de retenue est fermé, le débit du canal correspond au débít d'équipement et la retenue est à son niveau normal. Sur la gatuche de la pholo, on aperçoit l'exutuire de l'Embroye dans le canal. 
importante a nécessité l'établissement de guideaux (sur la rive gauche de la retenue en amont de la prise, sur la rive droite du canal en aval de celle-ci), ce qui a permis d'économiser des endiguements insubmersibles de grande hauteur qui eussent été nécessaires pour épouser les formes hydrauliques optimales de la prise.

A Baix-Le Logis Neuf, la courbure était nettement trop faible et il en résultait les inconvénients signalés plus haut. La mise au point de la prise a nécessité l'exécution de nombreux essais sur modèle. Finalement, les artifices adoptés (dérasement de la berge devant l'entrée, établissement d'épis) ont conduit à ramener le rayon de courbure à une valeur voisine de celle de Montélimar. Ici aussi, un seuil en béton d'une soixantaine de mètres de long facilite le creusement d'une fosse formant mouille artificielle et empêchant l'entrée des alluvions dans le canal.

Ajoutons que, dans ces deux dernières chutes, on a pu, sans précautions spéciales, profiter de la courbure modérée du lit pour rapprocher le barrage de la prise et améliorer ainsi l'évacuation du débit solide et l'efficacité des chasses malgré la grande hauteur de la retenue.

\subsection{Consignes d'exploitation en crues.}

Les consignes d'exploitation d'un aménagement en temps de crue doivent conduire à respecter un certain nombre de conditions de

\section{VITESSES MOYENNES DANS LE CANAL $\left|V_{1}\right| \mathrm{EN} \cdot \mathrm{m} / \mathrm{s}$}

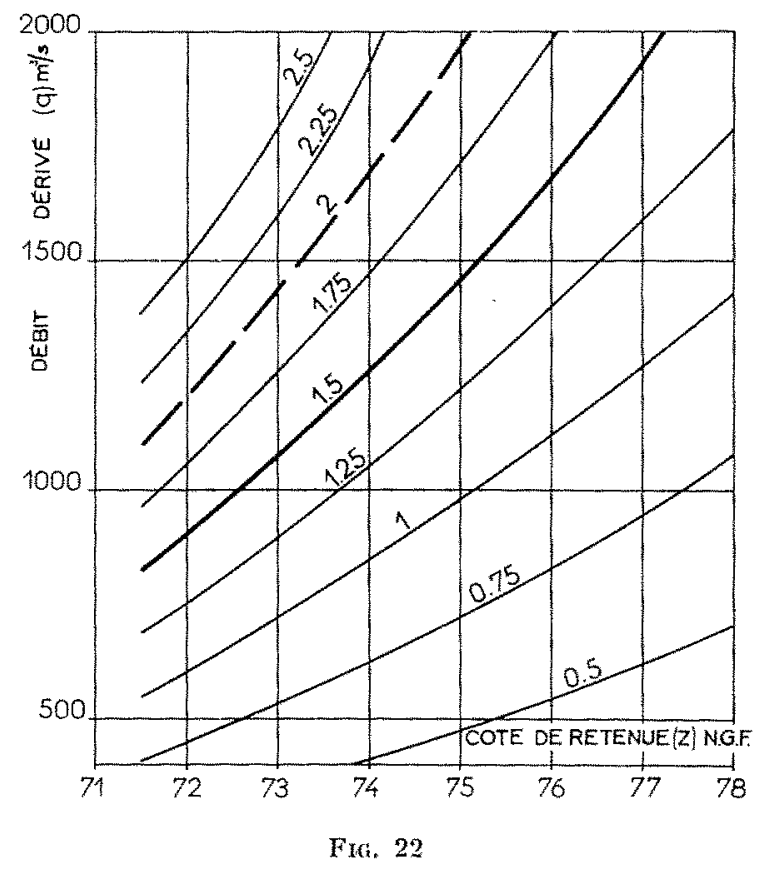

CONSGine D'exploitation de Montélimar.

Courbes des vilesses moyennes dans le caual (V1) en fonction du niveau de la retenue et du débit dérivé. vitesse que nous allons essayer de préciser cidessous.

En tous points de la retenue, de la prise, du canal de dérivation et du Rhône court-circuité, la vitesse dépend essentiellement de trois paramètres : $\mathrm{Q}$ (débit du fleuve), $q$ (débit dérivé), $\mathrm{H}$ (cote de la retenue au droit de la prise).

La mise au point de la consigne d'exploitation conduit notamment à définir pour chaque débit $Q$, les limites imparties aux deux autres paramètres pour qu'il n'en résulte pas d'inconvénients, tant en ce qui concerne l'exploitation de l'usine, le passage de la navigation que le cheminement du débit solide.

\subsubsection{Vitesses aU voisinage de La PRise.}

Examinons d'abord la situation au voisinage de la prise. Il faut veiller à ce que les vitesses au droit de la prise ou dans le canal de dérivation ne soient pas trop fortes:

- la vitesse moyenne dans le canal $\left(V_{1}\right)$ ne doit pas être trop grande, afin de ne pas gêner la navigation (13) et surtout d'éviter l'érosion des berges (qui sont seulement protégées à la partie supérieure par un léger revêtement de batillage) : sous ce rapport, il apparaît que $V_{1}$ ne doit en aucun cas dépasser $2 \mathrm{~m} / \mathrm{s}$ et il est souhaitable qu'elle reste inférieure à $1,5 \mathrm{~m} / \mathrm{s}$. De plus, eu égard au débit solide, il $\mathrm{y}$ a intérêt qu'elle soit comprise entre les vitesses limites $\mathrm{V}_{s}$ et $\mathrm{V}_{c}$ indiquées précédemment (fig. 22);

- de manière à éviter l'introduction de graviers dans la prise d'eau, la vitesse maximale $\left(V_{2}\right)$ devant l'entrée du canal doit également être limitée. Le modèle réduit a montré qu'il est souhaitable que $V_{2}$ n'atteigne pas $2,5 \mathrm{~m} / \mathrm{s}$ et qu'il est indispensable qu'elle ne dépasse pas $2,75 \mathrm{~m} / \mathrm{s}$; toutefois, pour les crues importantes de fréquence très faible $(Q$ de l'ordre de $6000 \mathrm{~m}^{3} / \mathrm{s}$ ), les valeurs ci-dessus peuvent sans inconvénients majeurs être portées respectivement à $2,75 \mathrm{~m} / \mathrm{s}$ et $3 \mathrm{~m} / \mathrm{s}$ (fig. 23 ).

Le respect de ces deux conditions peut conduire, en période de crues, à réduire le débit dérivé $q$ à une valeur inférieure à $q_{e}$.

(13) Dans les parties du fleuve non aménagées, les bateaux circulent pour des débits $Q$ compris entre l'étiage (dépassé $355 \mathrm{j} / \mathrm{an}$ ) et la crue dépassée $10 \mathrm{j} / \mathrm{an}$. Moyennant certaines précautions, ils peuvent naviguer sur certains tronçons pour des crucs correspondant à une fréquence $1 \mathrm{j} / \mathrm{an}$.

Pour les débits extrêmes, les bateaux sont en général moins gènés par les vitesses excessives que par l'insuffisance des mouillages ou des tirants d'air. Dans les canaux, on a cependant fixé à $2 \mathrm{~m} / \mathrm{s}$ la vitesse maximale à ne pas dépasser en exploitation normale pour les besoins de la navigation, alors que la flotte actuelle doit souvent s'accommoder de ritesses supérieures a $4 \mathrm{~m} / \mathrm{s}$ dans les parties non aménagées du fleuve. 

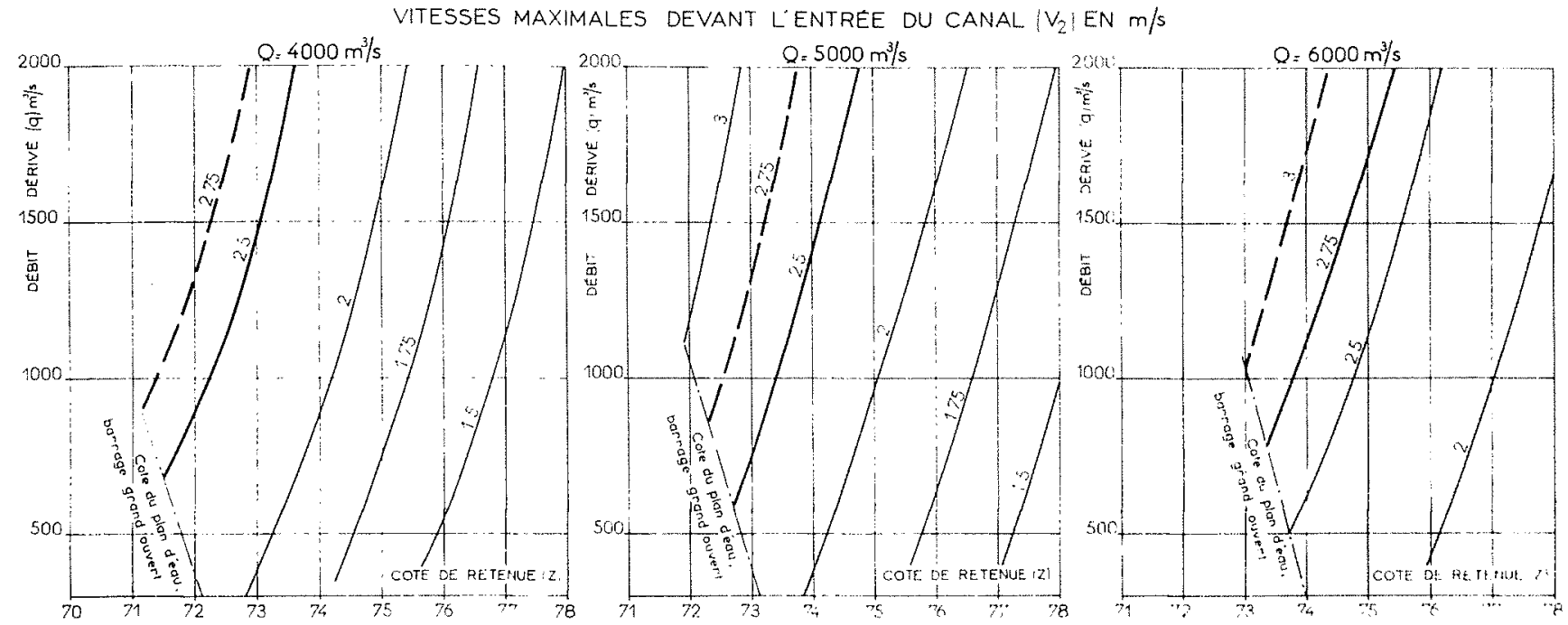

Fig. 23

Consigne d'exploitation de Mon'télimar.

Courbes des vitesses maximales devant l'entrée du canal (v2) en fonction du nireau de la retenue et du débit dérivé.

\subsubsection{Vitesses DANS LA RETENUE.}

Examinons maintenant, pour un débit donné $Q$ du fleuve la situation des vitesses moyennes $\mathrm{V}_{r}$ dans les différents profils en travers de la retenue situés en amont de la prise. Elles dépendent :

- de la position amont ou aval du profil dans la retenue;

- du débit dérivé $q$ (en raison du remous d'abaissement provoqué par la dérivation);

- et du niveau $\mathbf{H}$ de la retenue au droit de la prise, niveau qui peut être le niveau maximal de retenue $H_{m}$, ou un niveau abaissé $\left(H_{n}\right.$ ou $\mathrm{H}_{0}$ ).

Pour chaque situation d'exploitation (caractérisée par $\mathrm{Q}, \mathrm{H}$ et $q$ ), on pent distinguer dans la retenue les zones suivantes, en fonction de la valeur prise par la vitesse du courant $V_{r}$ : - zone d'envasement :

$$
\mathrm{V}_{r} \leqslant \mathrm{~V}_{\mathrm{s}}
$$

- zone sans charriage ni envasement:

$$
\mathrm{V}_{\mathrm{s}}<\mathrm{V}_{\mathrm{r}}<\mathrm{V}_{\mathrm{v}}
$$

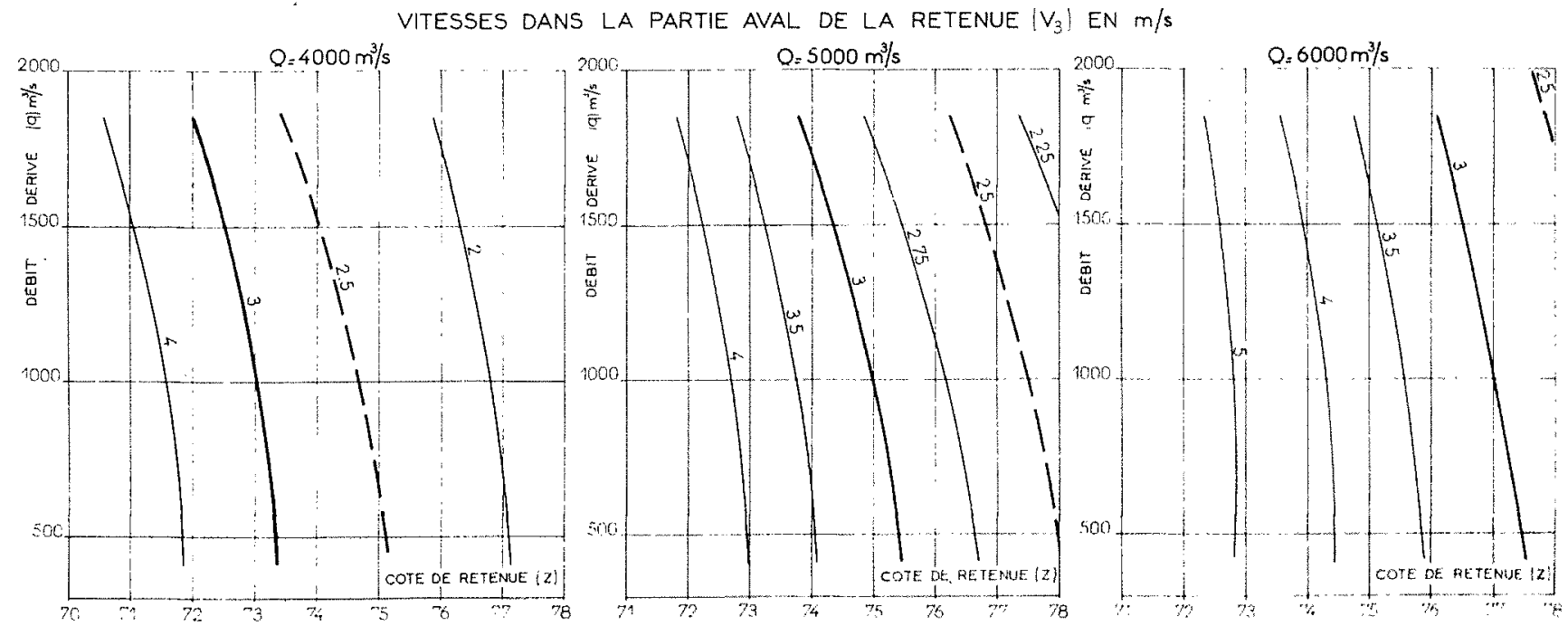

Fig. 24

Consigne n'explotitation dE Montélimar,

Courbes des vitesses moyennes dans la partie aval de la retenue (V3) en fonction du niveau de la retenue et du débit dérivé. 
- zone de charriage généralisé :

$$
\mathrm{V}_{r} \geqslant \mathrm{~V}_{e} \text {. }
$$

Il y a lieu d'examiner de mème la situation du troncon compris entre prise et barrage où la vitesse moyenne $\mathrm{V}_{r}$ dépend de $\mathrm{H}$ et de $\mathrm{Q}-q$.

En modifiant la valeur des parametres sur lesquels il est possible d'agir (H et $q$ ) pour un mème débit $Q$ du fleuve, on peut selon le cas, éviter ou favoriser le dépôt des matériaux fins, mobiliser les matériaux grossiers de l'amont de la retenue sans qu'ils puissent dépasser la prise (transit des matériaux de l'amont vers l'aval de la retenue), enfin évacuer tous les matériaux grossiers en aval de la prise on du barrage (chasse).

Il $y$ a lieu de veiller notamment aux points suivants :

- la vitesse dans la partie amont de la retenue (où se déposent généralement les graviers charriés par les débits moyens du fleuve)

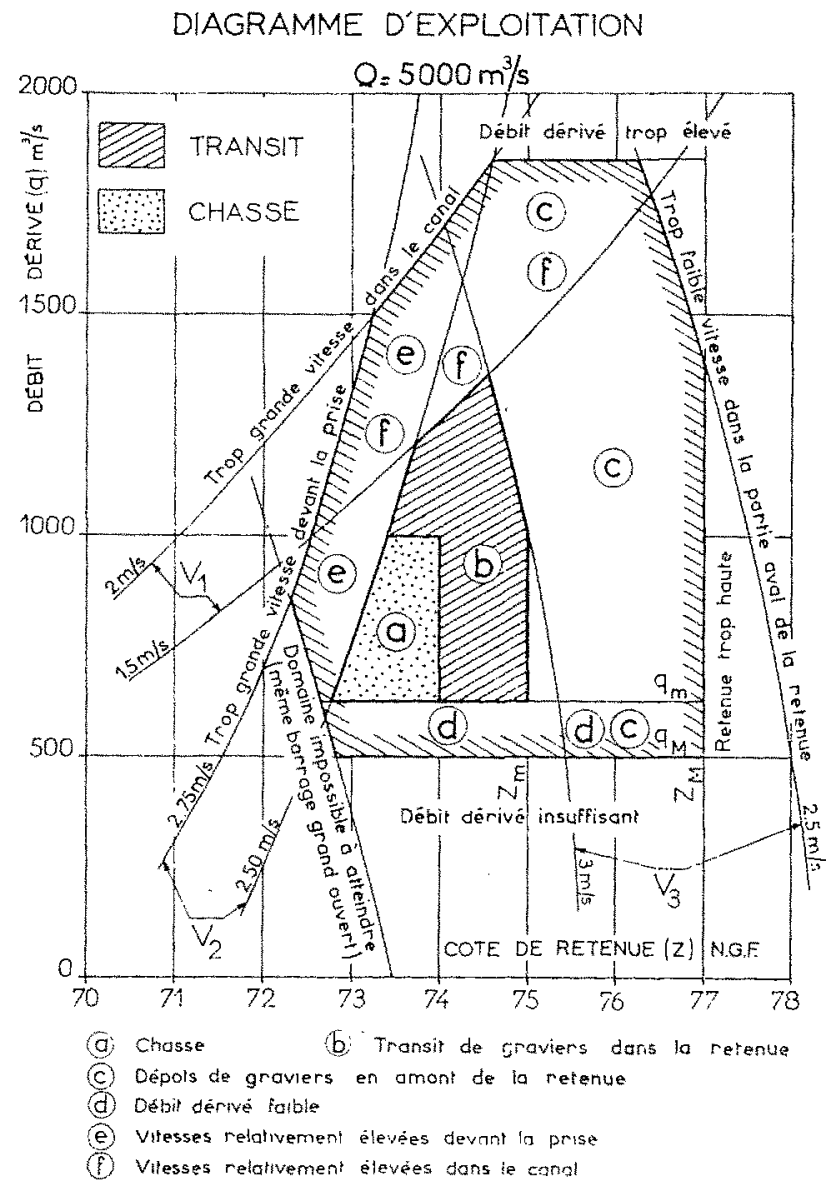

Fig. 25

Constge D'Explotitation de Montélimar.

Construction $d u$ diagramme donnant les differents domaines d'exploitation en fonction du niveau de la retenue et du débit dérivé (exemple de construction dans le cas du débit du Rhốne $\mathrm{Q}=5000 \mathrm{~m}^{3} / \mathrm{s}$ ). doit être suffisante pour mobiliser ces dépôts (régimes de transit et de chasse);

- la vitesse dans la partie aval de la retenue doit ĉtre assez grande pour entrainer vers le barrage (et en aval), les matériaux qui arrirent ou se sont déposés dans cette partie de la retenue (régime de chasse).

Pour que les chasses soient efficaces, il faut notamment que la vitesse dans la partie aval de la retenue $\left(V_{3}\right)$ soit supérieure à $2,5 \mathrm{~m} / \mathrm{s}$; il est mème préférable qu'elle dépasse $3 \mathrm{~m} / \mathrm{s}$ (fig. 24 ).

Lientrainement d'es graviers vers l'aval de l'aménagement est facilité d'autant plus que la cote de retenue est plus basse et que le débit dérivé est plus faible. Mais les nécessités de l'exploitation conduisent à limiter les chasses en nombre et en durée. En effet, l'abaissement de la retenue provoque une certaine perte de production à l'usine et entrave la circulation des bateaux (vitesses trop fortes, tirant d'eau diminué). La diminution du débit dérivé agit sur la production de l'usine de manière encore plus importante. Pour ne pas arrêter complètement la centrale, on est amené à fixer une valeur minimale $q_{0}$, au débit dérivé. Cette valeur, qui est en général de l'ordre de $500 \mathrm{~m}^{3} / \mathrm{s}$, est portée, en période de crue à $Q / 6$ ou $Q / 8$ selon les chutes.

\subsubsection{Consigne DE CHASSE PROpre A CHAQUE CHUTE.}

Le report sur un même graphique des différentes conditions énoncées ci-dessus (conditions matérialisées par les courbes représentant la liaison des divers paramètres entre eux) permet de délimiter, pour chaque aménagement, des domaines correspondant à des situations d'exploitation déterminées.

A titre d'exemple, la figure 25 donne la construction du diagramme d'exploitation pour l'aménagement de Montélimar (retenue haute) dans le cas d'une crue de $5000 \mathrm{~m}^{3} / \mathrm{s}$. Le domaine indiqué par un semis de points (situation de chasse) procure des conditions d'exploitation convenables pour une crue de longue durée, ou pour une crue survenant alors que la retenue comporte déjà un certain engravement; le domaine indiqué par des hachures serrées (situation de transit) convient pour une crue de courte durée et une retenue non engravée; le domaine limité par des hachures espacées correspond à des conditions d'exploitation sans doute possibles, mais qu'il est préférable d'éviter pour les différentes raisons indiquées sur le graphique. L'exploitation hors de ces domaines doit être rigoureusement proscrite.

La figure 26 permet de comparer, pour trois 


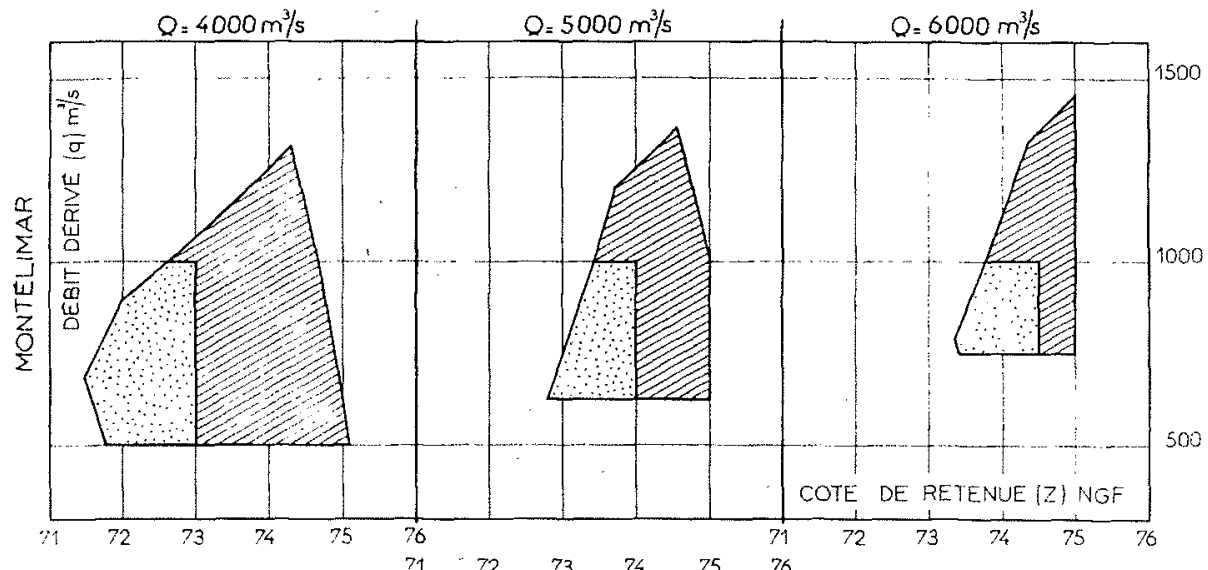

FIG. 26

CONSIGNES D'EXPLOITATION DES GHUTES

Comparaison des diagrammes d'exploitation des chutes de Montélimar et Baix-Le Logis Neuf (régime de chasse et régime de transit), pour trois valeurs du débit du Rhône $\mathrm{Q}$ $\left(4000,5000\right.$ et $\left.6000 \mathrm{~m}^{3} / \mathrm{s}\right)$.

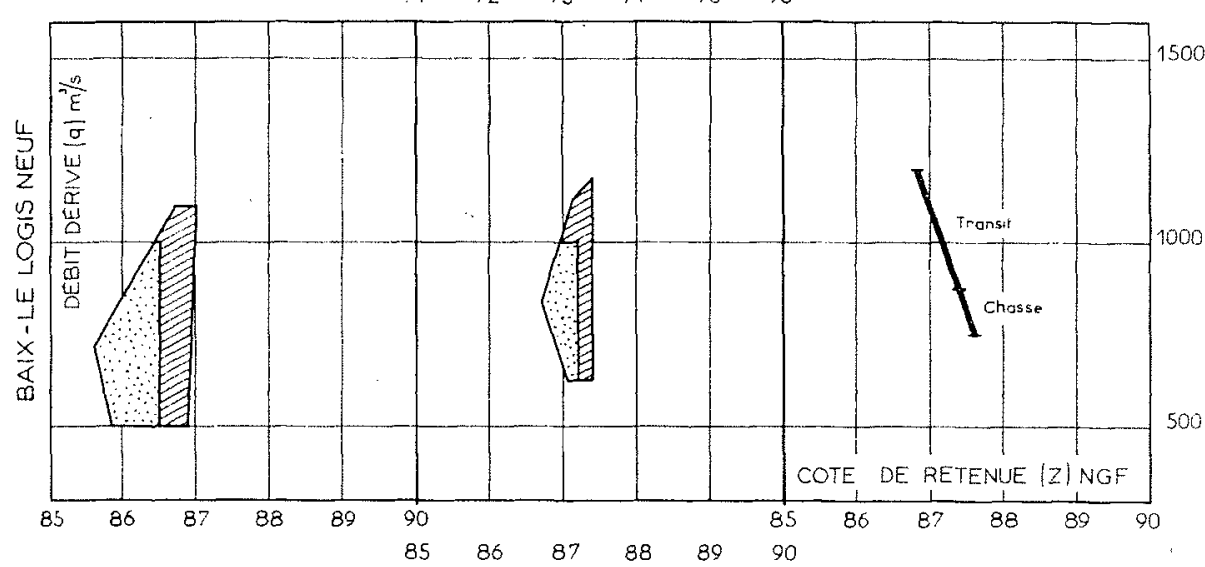

Regime de chasse regime de transit des materiaux

Fig. 27

Explortation de la chute de BaIX-Le Logrs Neuf

Courbes donnant, en fonction du débit du Thlône (Q) : - le niveau de la retenue (au droit de la prise d'eau), - le débit dérivé $(q)$,

- le rapport $q / Q$,

pour différents rêgimes d'exploitation : exploitation normale, régime de chasse, retenue maximale et débit dérivé maximal (ce dernier régime, purement théorique, ne tient pas compte des phénomènes liés au débit solide : il correspond aux niveaux maximaux admissibles pour les riverains et à un débit dérivé constant égal au débit d'équipement).

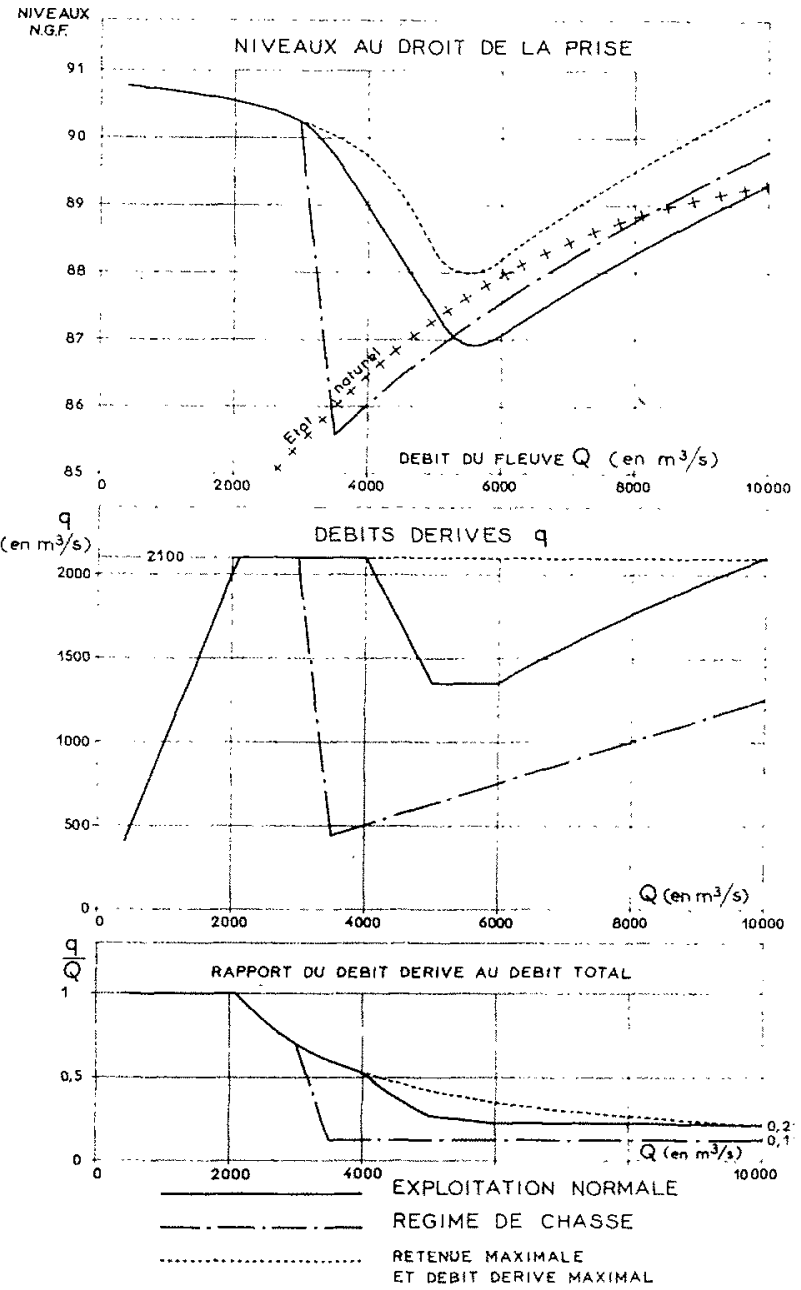


débits $Q$ de crue, les domaines d'exploitation obtenus pour les aménagements de Montélimar et Baix-Le Logis Neuf (seules la situation de chasse et la situation de transit sont représentées).

Grâce à ces graphiques, il est possible de définir une consigne d'exploitation indiquant pour chaque débit $Q$ du fleuve, la valeur optimale à donner au débit dérivé $q$ et au niveau de retenue H. En réalité, on est amené à définir : une con- signe normale d'exploitation (voir fig. 27) qui respecte au maximum la production de l'usine; une consigne occasionnelle plus sévère (consigne de chasse) dans laquelle la production de l'usine est notablement réduite pendant certaines crues, dont la fréquence ne dépasse pas en moyenne un ou deux jours par an.

Pour juger de l'efficacité des consignes mises au point, nous avons reporté sur un même graphique (fig. 28) les vitesses moyennes au droit
BAIX-LE LOGIS NEUF

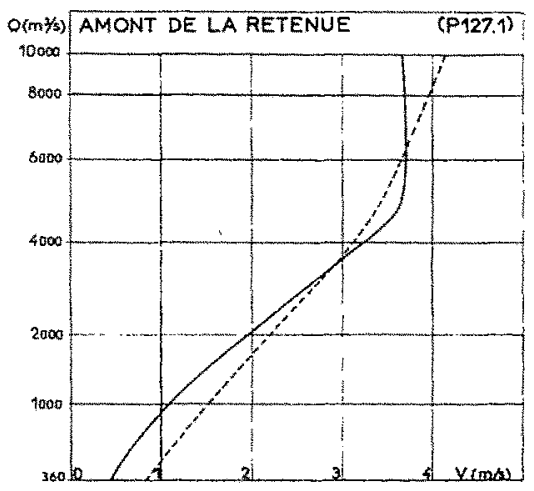

$Q(m / s)$ ENTRE PRISE ET BARRAGE (P135.6)

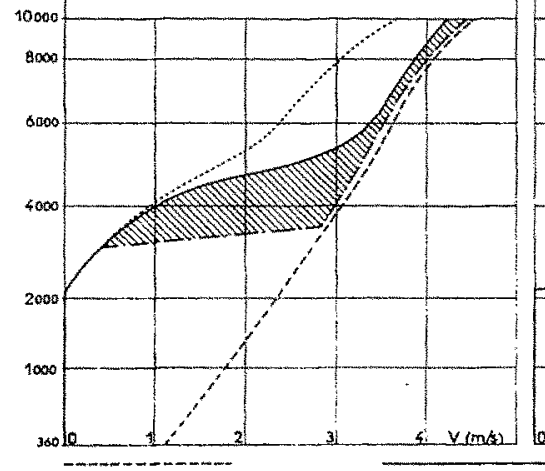

STAT NATURE!
EXPLOTATION NORMALE
VITESSES MOYENNES
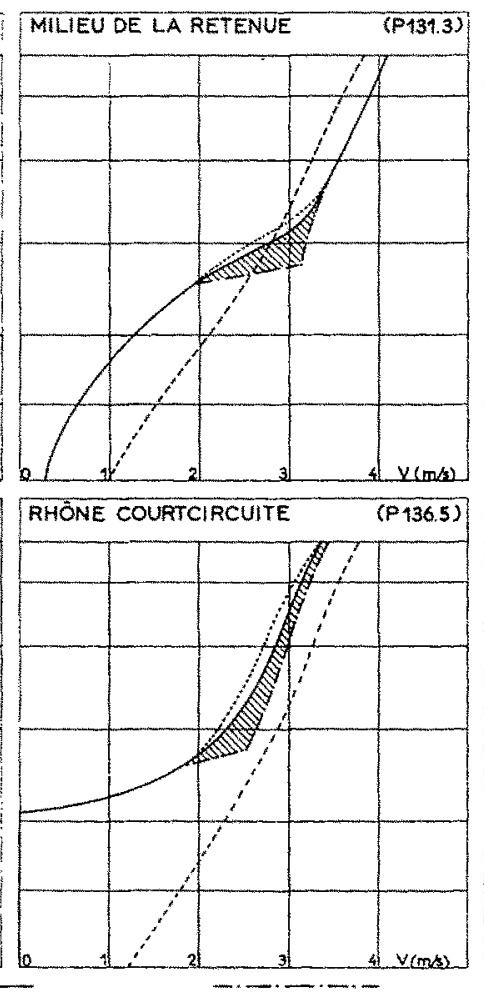

REGIME DE CHASSE
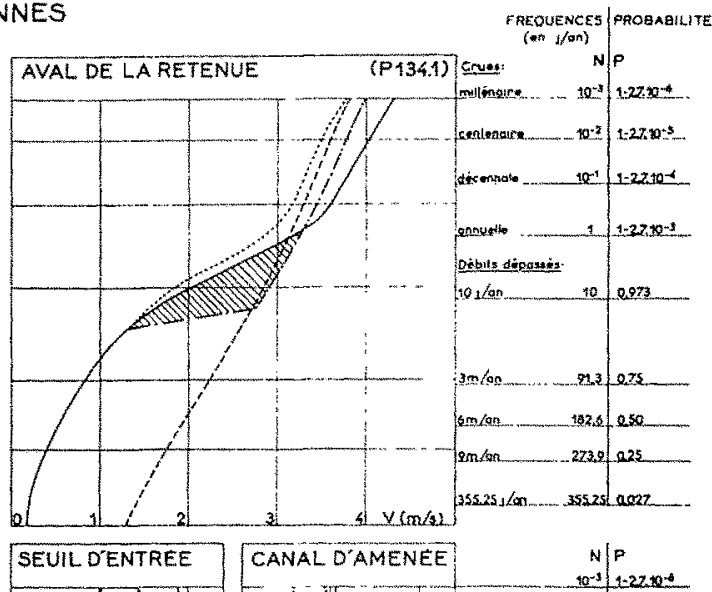

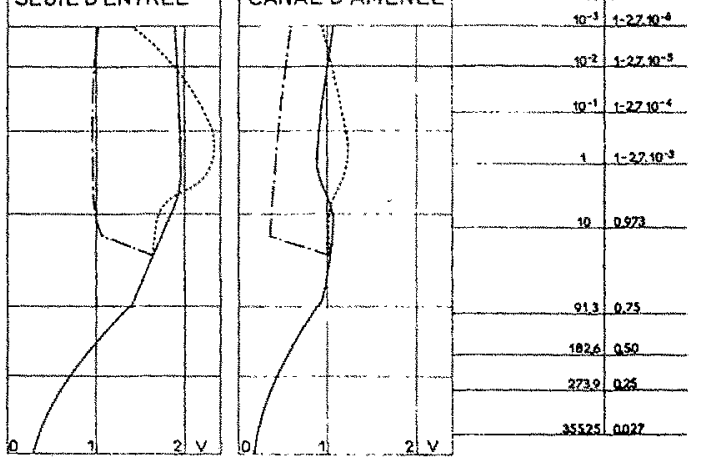

RETENUE MAXIMALE ET DEBIT DERIVE MAXIMAL

Fra. 28

Explottation de la Ghute de Baix-Le Logis Neur.

Courbes donnant la vitesse moyenne, en quelques points earacteristiques de l'nménagement, en fonction du debit du Rhône $(Q)$, pour différentes situations : état naturel (avant réalisation de l'aménagement), exploitation normale, régime de chasse, retenue maximale avec débit dérivé maximal (ce derniel cas, purement théorique, est explicité dans la légende de la figure 27 ).

les domaines hachurés sont ceux pour lesquels la vitesse en régime de chasse est supérieure à la vitesse en exploitation normale. On constate notamment que :

- entre la prise d'eau et le barrage, ainsi que dans le Rhône courtcircuité, la vitesse est plus faible après aménagement que dans l'état naturel; mais le régime de chasse accoit la vitesse par rapport a la situation de retenue normale;

- dans la partie amont de la retenue, le régime de chasse ne se distingue pas de la retenue normale;

- dans la partie mediane de la retenue, les chasses accroissent la vitesse pour les débits compris entre 3000 et $5500 \mathrm{~m}^{3} / \mathrm{s}$ environ; pour les débits supérieurs, le régime de chasse ne se distingue pas de la retenue normale;

- dans la partic aval de la retenue, les chasses accroissent la vitesse pour les débits compris entre 3000 et 5500 m ${ }^{3} / 5$ environ; elles la réduisent pour les débits supérieurs (mais, pour ces débits, la vitesse reste plus forte que dans l'état naturel); enfin, sur le seuil d'entrée et dans le canal, les chasses réduisent notablement la vitesse par rapport à la situation
de retenue normale. 
Frr. 29

VITESSES MOYENNES CLASSÉES DANS LA RETENUE de BaIX-Le Logis NeUf.

Courbes donnant en fonction du débit du Rhône $(Q)$, les vitesses moyennes dans la retenue (pour différentes situations : état naturel, retenue normale, régime de chasse).

Ces vitesses sont classées d'après les longuears des tronçons $d u$ fleuve où elles règment (à comparer avec la fig. 14).
RETENUE DE BAIX LE LOGIS NEUF

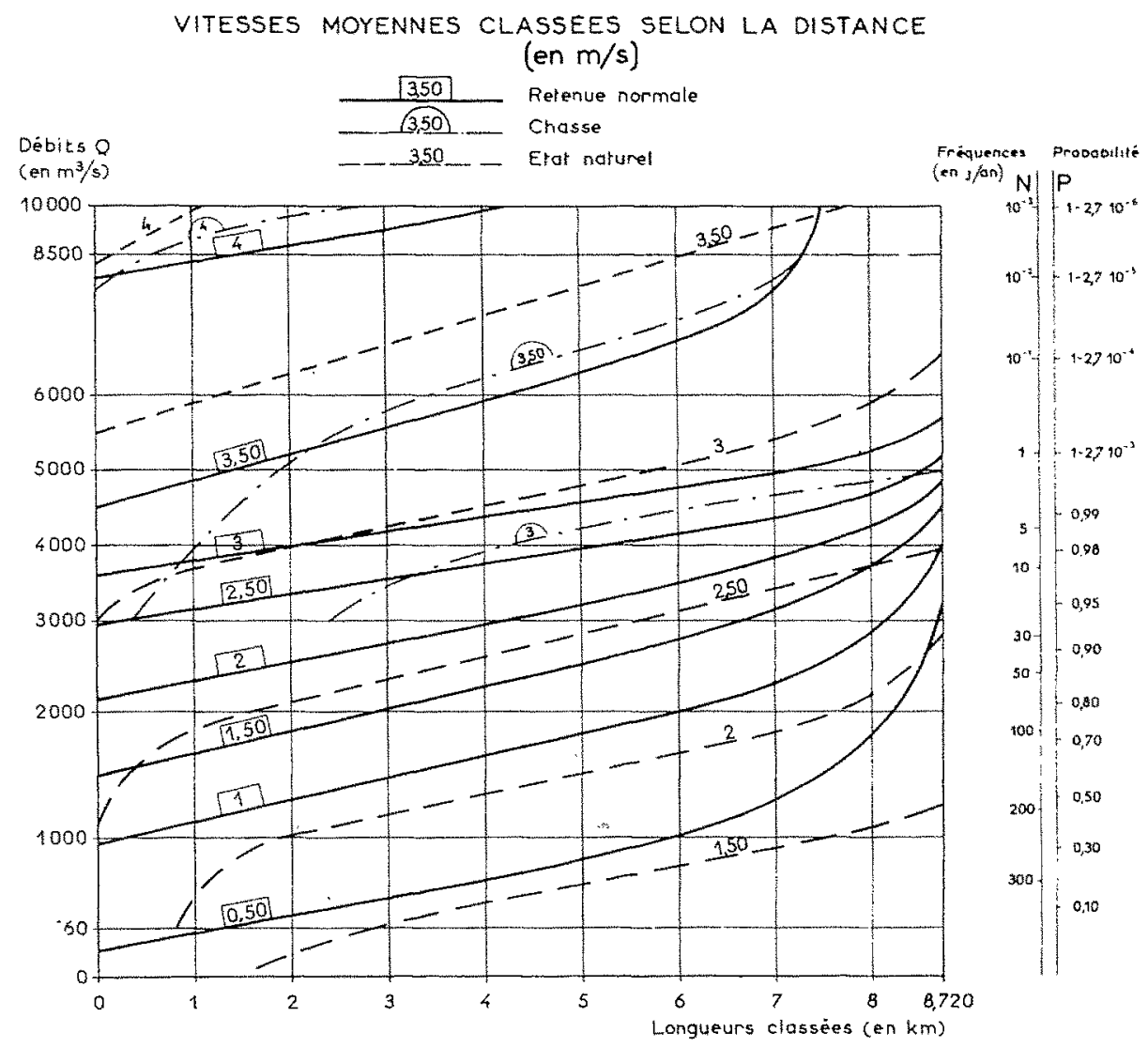

d'un certain nombre de profils en travers caractéristiques de l'aménagement de Baix-Le Logis Neuf, soit dans l'état naturel du fleuve, soit après aménagement. La consigne normale a déjà pour effet de réduire notablement les vitesses au droit de la prise et dans le canal, et d'augmenter dans la retenue les vitesses favorisant l'évacuation du débit solide. En situation de chasse, cet effet se trouve encore renforcé, sauf dans une partie très limitée de la retenue où les vitesses dépassent néanmoins $3 \mathrm{~m} / \mathrm{s}$ pour les débits supérieurs à $6000 \mathrm{~m}^{3} / \mathrm{s}$.

On peut aussi rassembler en un document unique les renseignements concernant les vitesses dans la retenue, classées en fonction de la fréquence et des distances d'application (fig. 29).

De tels graphiques permettent également d'étudier la rentabilité des chasses d'entretien, en comparant le prix de l'énergie perdue à l'usine avec celui des dragages qu'une mauvaise évacuation des graviers dans la retenue aurait nécessilés.

L'exploitation des retenues basses conduit à des consignes analogues aux précédentes. Si l'évacuation des dépôts dans la retenue est plus facile à assurer, la proteclion de la prise contre l'introduction des graviers nécessite par contre des précautions plus grandes. Nons avons dil que la prise de Donzère doit ètre, de ce point de vue, exploitée avec prudence. Lors de la crue de février 1955, époque à laquelle les consignes d'exploitation n'étaient pas encore parfaitement précisées, la retenue a été exploitée avec un niveau élevé et le débit dérivé a été maintenu à son maximum. Il en est résulté un engravement relativement important devant les seuils, mais les graviers n'ont pas pénétré dans la prise. S'i] devait continuer à s'engraver, par suite d'une exploitation trop audacieuse, le seuil usinier, malgré ses formes étudićes, ne pourrait plus joner le rôle qui lui est assigné pour la protection de la prise. Des chasses extrêmement sévòres ou même des dragages seraient alors nécessaires pour rétablir la situation.

Sans vouloir rendre compte ici des observatons très détaillées qui ont été faites sur le cheminement et le dépôt du débit solide dans nos aménagements et en particulier dans les retenues (nous en suivons l'évolution avec un soin extrême depuis plus de dix ans), nous nous con- 
tenterons d'indiquer que les consignes d'exploitation qui ont été mises au point ont permis de maintenir les prises libres de toute introduction de gravier. Les dépóts solides dans les retenues ont été limités à des volumes très modestes [25], que les chasses permettent d'ailleurs de mobiliser sans le secours de dragages. Seuls doivent être dragués quelques dépôts inévitables de matières en suspension dans les parties du canal de dérivation oir les vitesses sont faibles ou nulles (en particulier l'entrée des garages de navigation).

\subsubsection{Exploitation D'Une cascade de chutes.}

L'exploilation d'une série de chutes en cascade pose une autre catégorie de problèmes que nous n'avons pas encore abordée dans notre exposé. Malgré toutes les précautions prises, l'endiguement des retenues, en supprimant une partie des champs d'inondation naturels, tend à accélérer la propagation des crues et peut conduire à en majorer le débit de pointe. Le Rhône courtcircuité tend à provoquer un effet inverse, car son lit est soulagé du débit dérivé yers l'usine; mais ces deux effets ne se compensent pas forcément. D'autre part, les consignes d'exploitation normales ou exceptionnelles propres à chaque chute conduisent, pour certaines crues, à abaisser le nivean des retenues et, par consé- quent, à vider une partie des volumes accumulés. La vidange simultanée de plusieurs réservoirs tendrait évidemment à majorer notablement les crues en aval, malgré la faible capacité utile de chacun d'eux.

L'exploitation, en période de crues, d'une cascade d'aménagements pose done une série de problèmes délicats, qui relèvent plus de la recherche opérationnelle que du calcul élémentaire ou du modèle réduit. Il en serait de même de l'étude d'une exploitation rationnelle des chutes au point de vue de la puissance de pointe.

Nous procédons actuellement à la mise au point d'une consigne d'ensemble relative à l'écoulement des crues dans les aménagements successifs. Cette étude complexe s'effectue avec l'aide d'un «modèle mathématique», qui n'est autre qu'un programme comportant toutes les caractéristiques du lit et des ouvrages ainsi que les conditions de fonctionnement propres à chaque chute. Ce programme permet de représenter, sur un ordinateur électronique, l'écoulement des crues à travers la chaine des aménagements, et de comparer les niveaux et débits obtenus à ceux de l'écoulement naturel.

Grâce à ce modèle, on peut harmoniser entre elles les consignes propres à chaque aménagements en vue de satisfaire au mieux tous les intérêts en cause.

\section{3. - LE BLOC USINE-DECHARGEUR-ECLUSE}

Dans les chutes du Bas-Rhône, on a trouvé avantagenx de grouper l'usine, le déchargeur et l'écluse en un bloc unique : non seulement, le remplissage et la vidange du sas s'en trouvent facilités, ainsi que les conditions de navigation dans les garages de l'écluse, mais on aboutit aussi à un ensemble d'ouvages de forme ramassće qui permet d'importantes économies, tant en ce qui concerne la conception des projets (services communs d'alimentation d'énergie électrique, d'air comprimé, réseau unique de drainage), la construction des ouvrages (chantier unique logé dans une même fouille et bénéficiant des mêmes movens d'exćcution ef de bétonnage) que pour toutes les charges de l'exploitation.

Du voisinage de l'usine et de l'écluse, il résulte cependant quelques inconvénients que nous allons analyser ci-dessous. On verra qu'ils ont pu être supprimés ou suffisamment atténués, au prix d'études sans doute délicates, mais sans ouvrages ou dépenses supplémentaires notables venant remettre en question le bilan de l'opération.

\subsection{Alimentation et restitution du bloc usine- déchargeur-écluse.}

\subsubsection{AlIMENTATION.}

Le tracé des berges du canal d'amenée et les formes d'entrée du bloc usine-déchargeur-écluse doivent être étudiés très soigneusement de manière à faciliter au maximum l'entonnement des eaux vers les pertuis de l'usine et du déchargeur ainsi que vers la prise de l'écluse, tout en évitant la formation de zones d'eaux mortes ou de courants traversiers générateurs de vortex. 


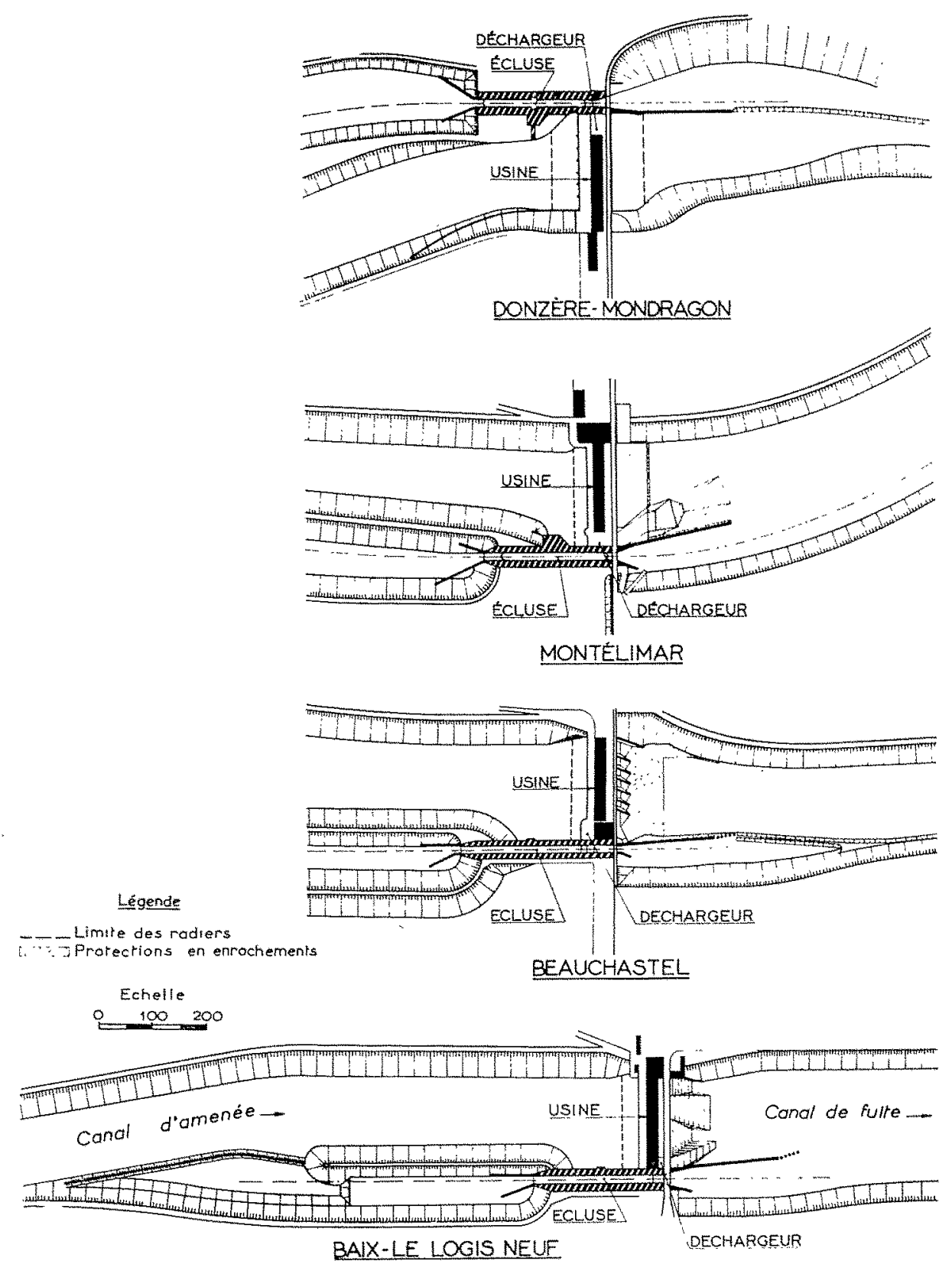

Fig. 30

Plans comparatifs des abords des ensembles nsine-dehargeur-écluse du Bas-Rhone.

Au droit du musoir de la digue séparant le canal usinier du garage de navigation (fig. 30), il faut également éviter que le courant empruntant le canal n'ait tendance à s'infléchir vers l'entrée du garage, car il en résulterait deux inconvénients principaux :

- les échanges d'eau, avec le garage de navigation, pourraient provoquer des envasements, favorisés par l'orientation des courants de densité;
- les courants traversiers, ainsi engendrés, pourraient entrainer les bateaux contre le merlon, à l'entrée du garage.

Le tracé des ouvrages a été mis au point après essais sur modèle réduit. Pour l'étude de l'écoulement au voisinage du branchement, on a procédé à de nombreuses chronophotographies en visualisant les courants à l'aide de confettis et en utilisant une maquette de bateau pour étudier les effets du courant (fig. 31). 


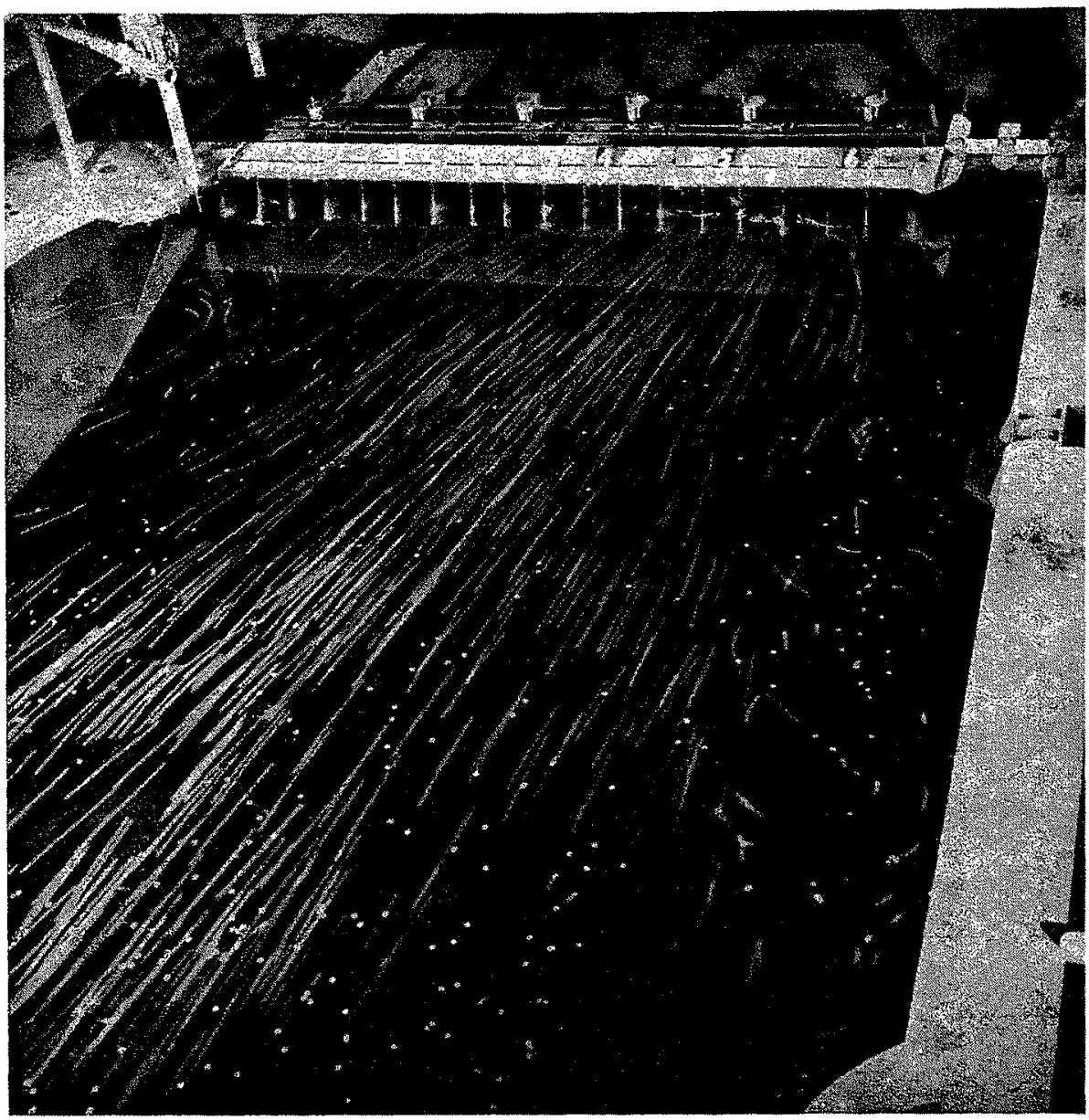

(Photo Neyrfim.)
Fia. 31

Etude sur modèle réduit des courants à l'amont de l'usine du Logis Neuf.
Il est apparu que, dans cette zone située à plusieurs centaines de mètres de l'entrée de l'écluse, les bateaux s'accommodent facilement de courants traversiers de plusieurs $\mathrm{dm} / \mathrm{s}$. N'ayant pas encore à ralentir, ils conservent en effet toute leur vitesse par rapport an courant et disposent ainsi des facultés de manœuvre nécessaires. Avec les formes qui ont été adoptées en définitive au voisinage du branchement, les courants traversiers ne dépassent jamais $0,25 \mathrm{~m} / \mathrm{s}$, et ont plutôt tendance à écarter les bateaux du musoir. D'autre part, on a pu éliminer presque complètement les échanges d'eau entre le bief usinier et le garage de navigation.

En définitive, les problèmes hydrauliques de l'amont ont été assez faciles à résoudre, du fait de la faible vitesse du courant $(1 \mathrm{~m} / \mathrm{s})$ et de la grande distance $(600$ à $800 \mathrm{~m})$ qui règne entre l'usine et l'entrée du garage de navigation. Grâce à des formes appropriées, on a pu éviter les guideaux ou les ouvrages spéciaux qui se sont révélés nécessaires à l'aval.

Par contre, les changements de régime de lusine entrainent dans le canal notamment, la formation d'ondes de translation dont certaines conséquences peuvent être gênantes: une étude très détaillée a été nécessaire, dont nous rendons compte ci-après.

\subsubsection{Restitution}

L'étude des problèmes posés à la restitution de l'usine s'est révélée beaucoup plus délicate que ceux de l'entrée, notamment par suite de la relative proximité $(150$ à $200 \mathrm{~m})$ entre la restitution de l'usine et l'entrée du garage aval de navigation. Un autre élément défavorable vient s'y ajouter : la vitesse du courant est notablement plus grande et plus inégalement répartie qu'en amont.

Alors qu'à Logis Neuf, la vitesse moyenne de l'écoulement dans le canal de fuite ne devrait pas dépasser $2 \mathrm{~m} / \mathrm{s}$, la vitesse à la sortie des groupes, (en moyenne : $2,43 \mathrm{~m} / \mathrm{s}$ ) atteint par endroits $6 \mathrm{~m} / \mathrm{s}$, car le débit est très inégalement réparti entre les deux pertuis de l'aspirateur $(74,6 \%$ sur R.G. et $25,4 \%$ sur R.D.), malgré les soins apportés par le turbinier. 
Cette distribution dissymétrique de la vitesse (voir fig. 45) donne lieu à des courants violents et à des mouvements tourbillonnaires locaux. II en résulte des pertes de charges notables par échange turbulent et même des risques d'érosion locale. Ces risques se sont d'ailleurs manifestés sur la berge rive gauche lors de la mise en eau de l'usine de Châteauneuf.

D'autre part, lorsque plusieurs groupes ne fonctionnent pas, la répartition du courant issu des turbines en service est aussi très inégalement assurée dans la largeur du canal. Du fait de la récupération de la quantité $V^{2} / 2 g$, les pentes peuvent s'inverser localement. Il se crée des contre-courants et des zones d'eau morte où s'établissent des rouleaux et courants de retour de grande amplitude, qui peuvent péném trer dans l'entrée du garage de navigation. IIs engendrent a leur tour des rouleaux secondaires dans la zone de contact entre l'écoulement du canal de fuite et les masses stagnantes du garage. Il peut en être de même lors du fonctionnement occasionnel du déchargeur.

Une étude très détaillée des formes aval a été nécessaire pour localiser les courants de retour et les zones d'eau morte dans le canal usinicr, et limiter au maximum l'ampleur et la vitesse des rouleaux. Ces derniers assureraient en effet un approvisionnement continu de matiores en suspension transportées par les eaux du flcuve, et ces matières se décanteraient dans les eaux calmes. De plus, les faibles courants, souvent instables, qui naissent à l'entrée du garage, peuvent, dans certaines conditions, provoquer une forte dérive des bateaux et rendre difficile, voire nême impossible, l'accès au garage aval ou à

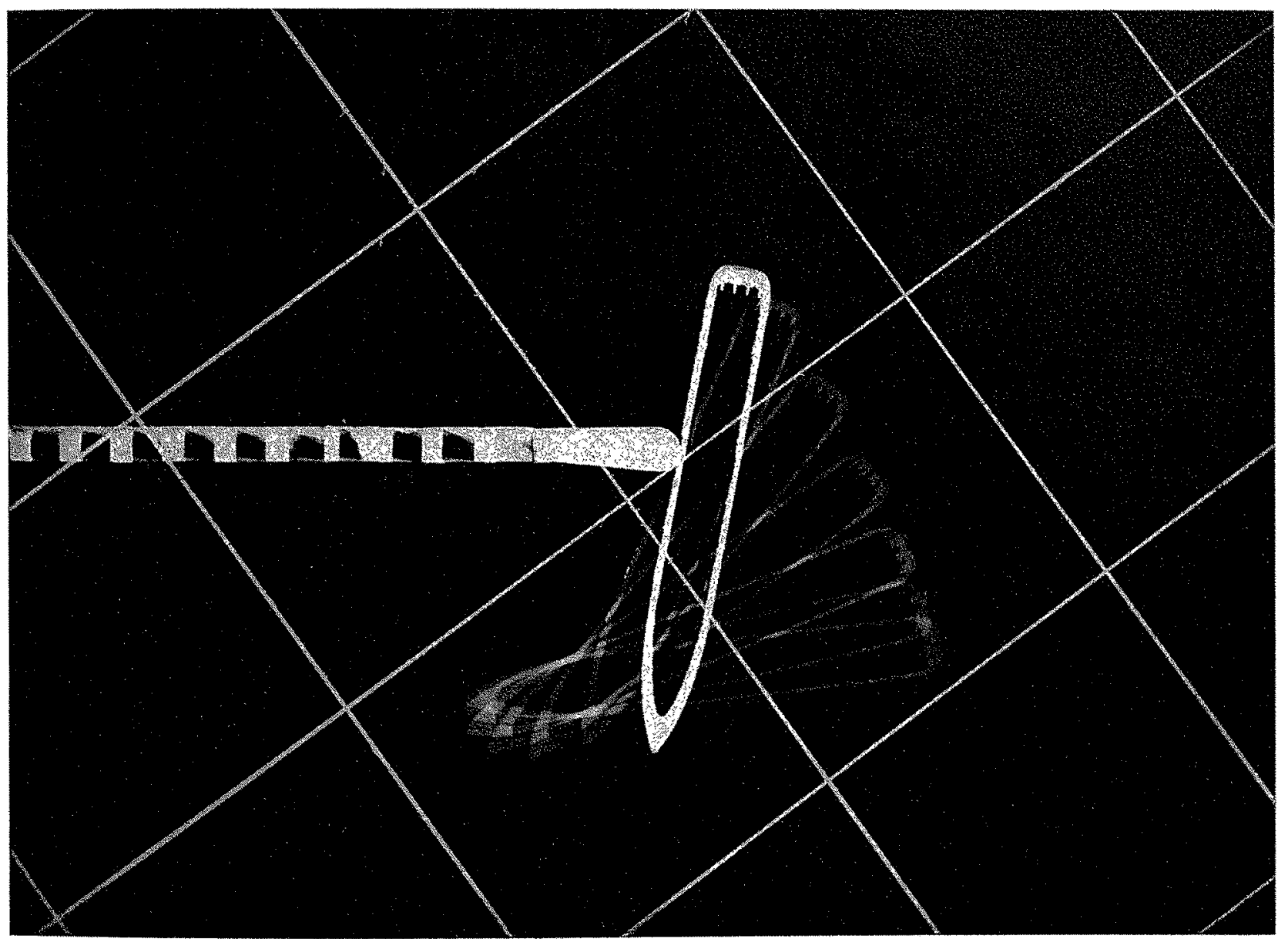

(Photo Noynflm,

Fid. 32

Conditions de navigation à l'ontrée du garage aval de Châteauneuf (premiere étude sur modele avant mise au point des formen défnítives). Chronophotographie d'un bateau ahandonné à lui-méme. 
l'écluse, notamment pour les longs convois qui, ralentissant à l'approche de l'écluse, sont difficiles à manœuvrer.

Avant que ces problèmes n'aient pu être complètement étudiés et résolus, on a constaté à Châteauneuf que certaines barques avaient tendance à ètre déportées accidentellement contre les ducs d'Albe ou le mur divisoir en rive gauche. Pour que ces inconvénients très exceptionnels ne se manifestent pas, l'étude et l'expérience ont montré qu'il est indispensable de limiter les courants traversiers éventuels à $20 \mathrm{~cm} / \mathrm{s}$ et souhaitable de les réduire à $10 \mathrm{~cm} / \mathrm{s}$ (fig. 32 ).

Il a été nécessaire de compléter les observations chronophotographiques concernant la vitesse et la direction des écoulements par une longue série d'essais mettant en évidence l'action du courant sur les trajectoires d'un bateau entrant dans le garage aval ou stationnant près de l'entrée. Comme les frottements, légèrement visqueux sur le modèle à $1 / 60$ utilisé, ne suivent pas rigoureusement la loi de similitude de Froude, les essais donnent d'ailleurs une vision un pen pessimiste des phénomènes. Les figures 33 et 34 montrent notamment le résultat de quelques essais réalisés à Logis Neuf avec la maquette d'un bateau de $70 \mathrm{~m}$ de long.

En définitive, l'étude des formes à donner aux ouvrages aval a été relativement longue et délicate. Le groupement de l'usine, du déchargeur et de l'écluse en un bloc unique facilite sans doute grandement le problème de l'alimentation et de la vidange des écluses, évite les notables courants longitudinaux qui régneraient sans cela dans les garages de navigation et conduit à des économies certaines sur l'ensemble des ouvrages.

Par contre, la sortie des turbines est séparée du garage aval par un mur divisoir que l'économie conduit à raccourcir au maximum $(180 \mathrm{~m})$; les difficultés hydrauliques évoquées plus haut seraient très facilement résolues si la longueur de ce mur était au moins deux à trois fois plus grande. Cependant, l'économie à attendre d'un raccourcissement $d u$ mur justifie pleinement l'ampleur des études délicates entreprises. A défaut de pouvoir agir sur les causes (dissymétrie dans la répartition des vitesses), il a fallu en limiter les effets en guidant judicieusement les courants néfastes, en les contrariant mutuellement, et en les freinant, grâce à la rugosité des fonds. Mais cette dernière, d'ailleurs génératrice de pertes de charge, ne peut agir que Iorsqu'un courant déjà notable s'est établi (fig. 30).

A Bollène, l'analyse des phénomènes relatifs à la navigation au voisinage de l'entrée du garage aval n'avait pas été menée de manière aussí complète. Le problème était d'ailleurs moins ardu que pour les aménagements ultérieurs (disposition favorable de l'écluse par rapport à la cour- bure du canal de fuite, et rapport de largeur plus important entre garage aval et canal de fuite, réduisant les dissymétries et facilitant l'entrée des bateaux). Les formes assurant un bon écoulement dans le canal ont été déterminées d'après un modèle comportant une répartilion uniforme des vitesses dans les aspirateurs. Le court mur divisoir $(150 \mathrm{~m})$ comportait des séries d'ouverture destinées à économiser le béton tout en favorisant l'amortissement des ondes de disjonction.

L'expérience a montré que le garage aval était soumis à un envasement relativement rapide et que la dissymétrie des vitesses et la dissipation de l'énergie à la sortie de l'usine engendrent non seulement des rouleaux qui peuvent être gènants dans certains cas, mais aussi des oscillations du plan d'eau, de faible amplitude (une dizaine de centimètres environ) et de fréquence 3 à $4 \mathrm{~s}$, préjudiciables au sommeil des équipages des bateaux stationnant contre le mur divisoir. Une représentation correcte de la distribution des vitesses opérée sur le modèle après la mise en eau de l'aménagement a permis de reconstituer les phénomènes et de trouver les moyens d'y remédier.

A Châteauncuf, la dissymétrie des vitesses à la sortie des aspirateurs tendrait à favoriser les courants sur la rive gauche. Mais du fait de la courbure du canal qui s'incurve précisément vers ce côté, et de sa largeur plus grande, les courants sont finalement relativement modestes et bien centrés.

Pour éviter la formation de rouleaux à l'entrée du garage aval, il apparaît nécessaire qu'une partie des courants soit dirigée vers l'extrémité du mur divisoir préalablement allongé $(180 \mathrm{~m})$. Cette condition s'est trouvée réalisée avec les formes de berges prévues. Un petit mur déflecteur a cependant été nécessaire pour éloigner les courants de la rive gauche, contre laquelle ils avaient tendance à coller. De plus, il a fallu prévoir une saignée dans le plafond du canal à la sortie du déchargeur, pour éloigner les courants sortant de cet ouvrage du mur divisoir contre lequel, sans précautions spéciales, ils atteindraient des vitesses trop fortes.

Au Logis Neuf, à l'inverse du cas précédent, le canal de fuite s'incurve vers la droite, la courbure étant toutefois plus faible et plus éloignée de l'usine: les conditions naturelles sont done moins bonnes.

Cependant, lors de l'exécution des fondations de l'usine, l'entrepreneur a été amené à exécuter, pour faciliter l'accès de ses camions au fond de fouille, deux rampes inclinées assez profondes, une sur rive droite, l'autre au tiers du chantier sur rive gauche. Ces rampes devaient normalement être remblayées après les travaux pour l'établissement du radier de pente uniforme qui 


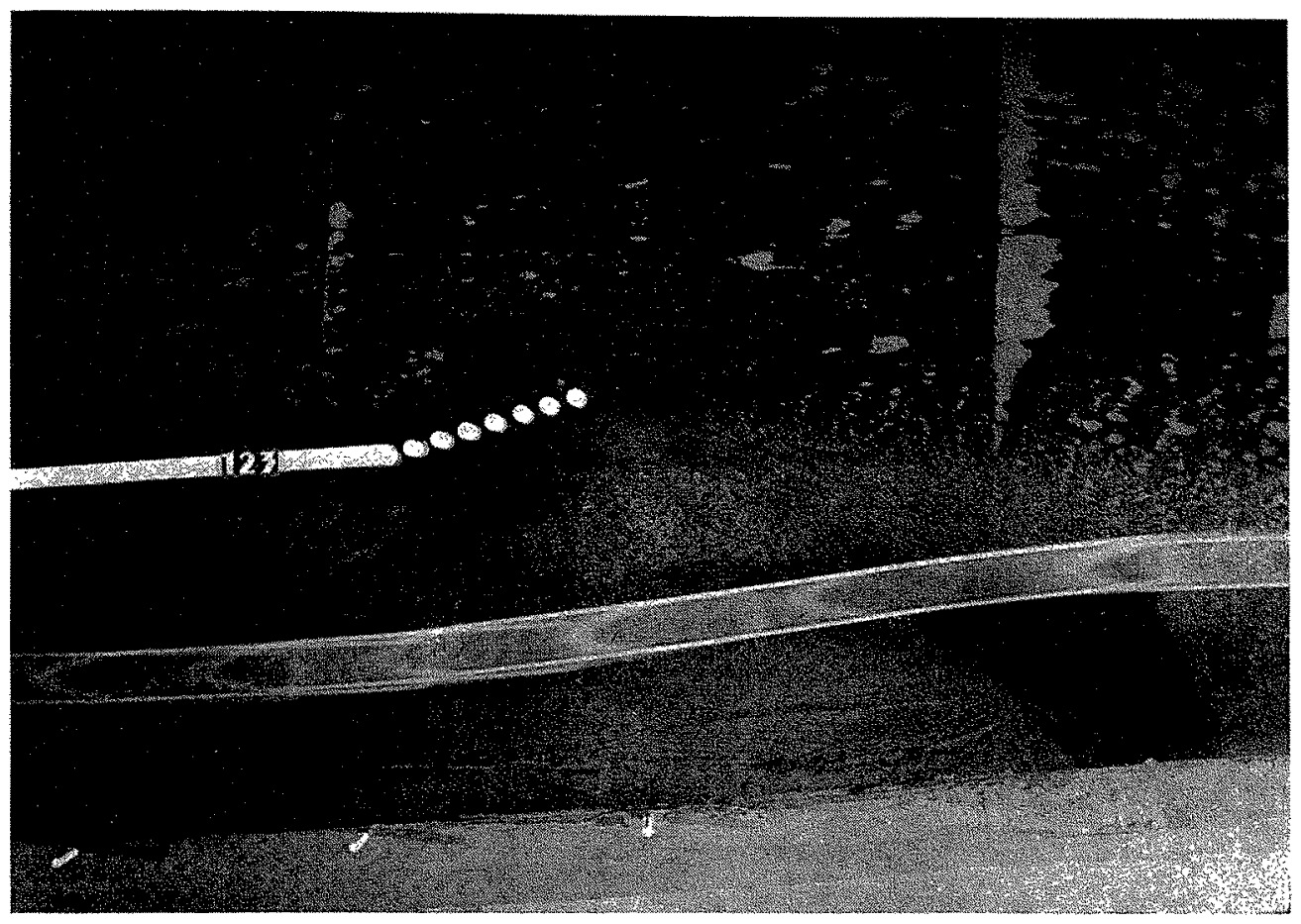

Fig. 33

(Photo Neyrfilm.)

Conditions de navigation à l'entrée du garage aval du Logis Neuf (étude sur modèle après mise au point des formes définitives). Chronophotographie d'un bateau laneé.

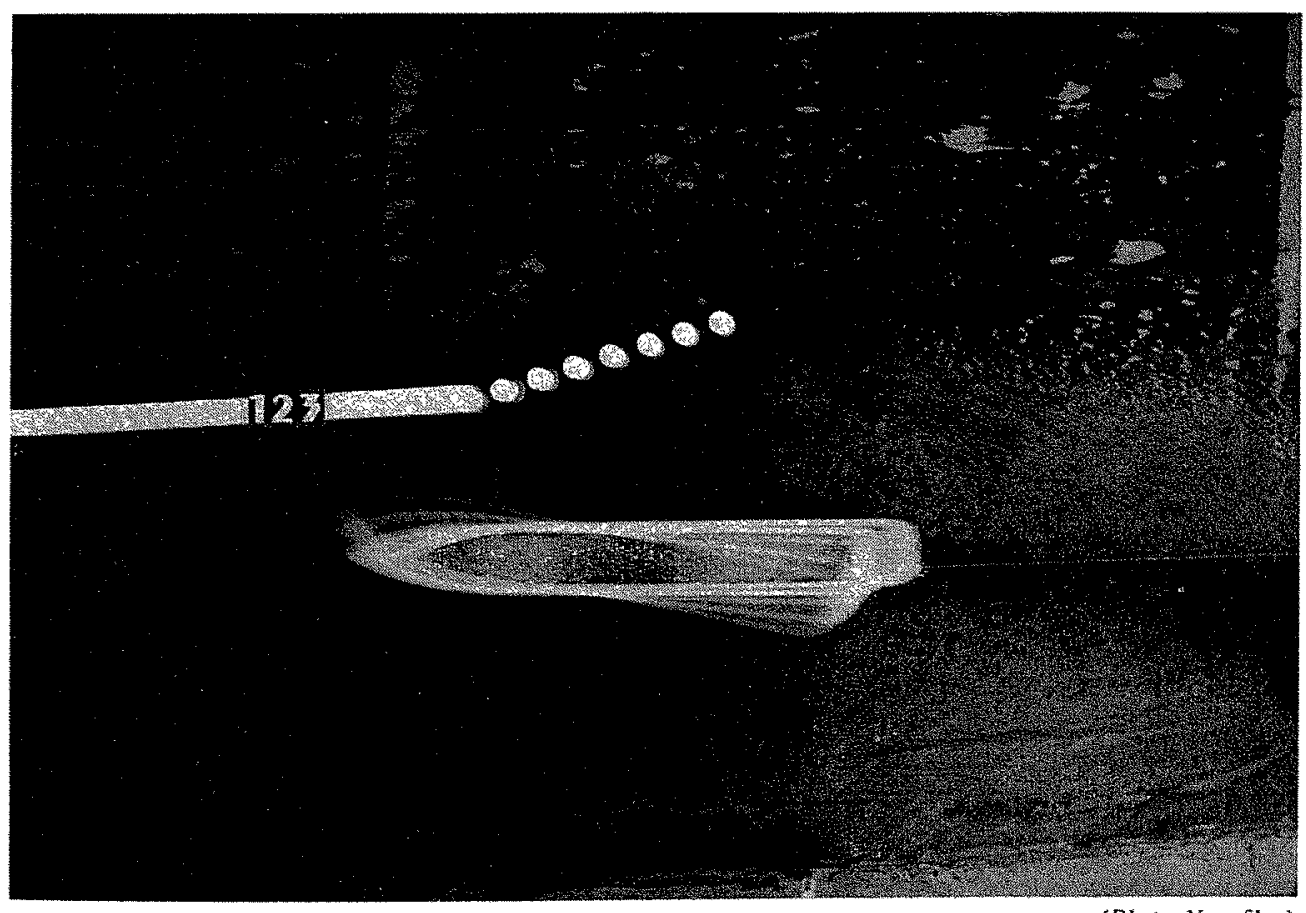

Fia. 34

(Photo Neyrfinm.)

Conditions de navigation à l'entrée du garage aval du Logis Neuf (étude sur modele après mise au point des formes définitives). Chronophotographie d'un batea arrêté. 
Fig. 37

Courants à l'aval de l'usine et de l'écluse du Logis Neuf. Vue d'ensemble de l'écoulement sur modèle réduit.

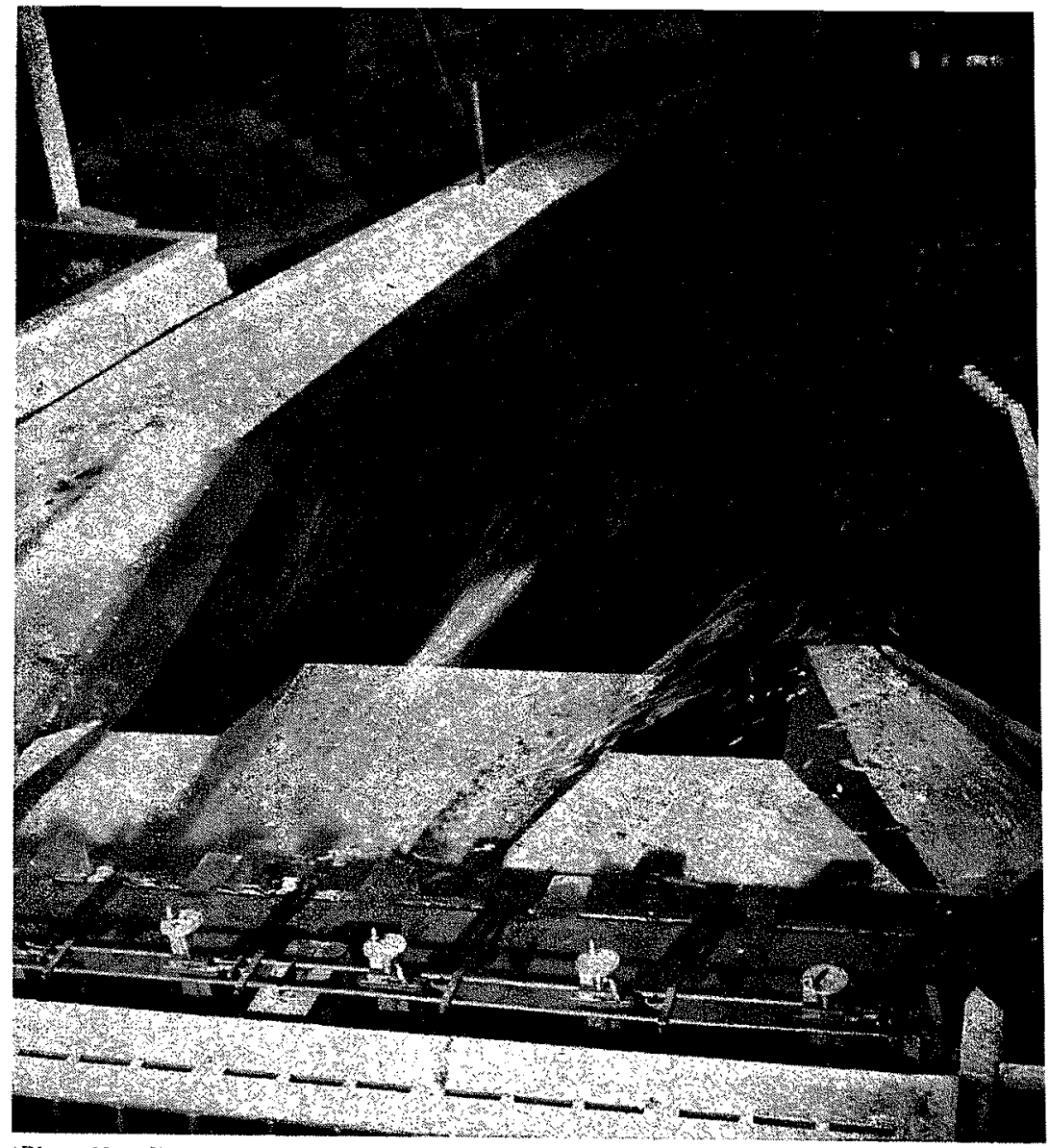

(Photo Neyrfilm.)

garage aval a été mené avec un soin extrême. Il est apparu que l'intensité et la direction des courants traversiers observés dépendent non seulement de la forme des ouvrages, mais aussi du nombre et de la position relative des groupes débitant dans l'usine (fig. 37). On n'a pas cherché à supprimer complètement les courants traversiers dans tous les cas de figures possibles, mais on s'est attaché à les supprimer ou à les réduire dans tous les cas qui peuvent se présenter en pratique.

Les soixante-trois combinaisons possibles correspondant à la pleine marche ou l'arrêt des groupes, ont toutes été essayées (fig. 38). L'étude a montré que les courants traversiers sont négligeables (moins de $7 \mathrm{~cm} / \mathrm{s}$ ) lorsque trois au moins des six groupes sont en service quelle que soit leur situation respective.

Pour un nombre de groupes en service inférieur à trois, les courants traversiers engendrés sont souvent négligeables; mais, dans quelques cas (huit combinaisons sur le total de soixantetrois), ils sont simplement faibles (de 15 à $20 \mathrm{~cm} / \mathrm{s}$ ). En fait, on ne se trouve jamais dans une telle situation en exploitation normale de la chute: même en période d'étiage, où le débit pourrait être turbiné par deux groupes seulement, le dispatching impose en elfet de répartir toujours la charge entre trois groupes. De plus, lors de la mise en service de l'aménagement, où l'on est amené à n'utiliser qu'un ou deux groupes sans avoir la latitude d'en choisir

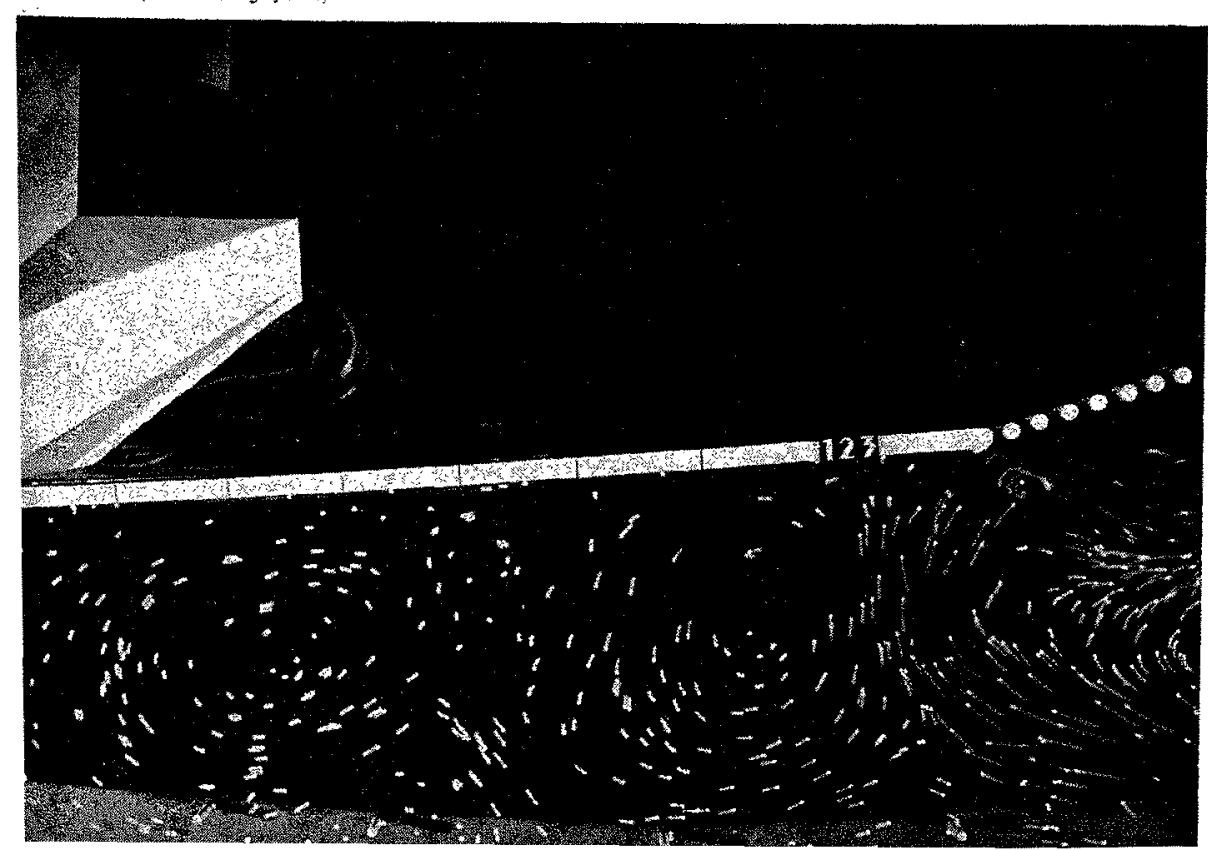

Fic. 38

Conditions de navifation à l'entré du garage aval de l'écluse du Logis Neuf. Chronophotographie des confettis sur le modele réduit. 
judicieuscment l'implantation, on a également la possibilité de supprimer les courants éventuels par l'utilisation du déchargeur, le débit disponible dans le fleuve étant alors surabondant.

Le tableau $V$ donne une récapitulation des différentes situations dont il vient d'être question.

A Beauchastel, le problème était plus difficile à résoudre pour deux raisons:

- à la sortie de l'usine (fig. 39), le canal s'incurve vers la rive droite, alors que les courants, comme nous l'avons vu, ont tendance à se diriger vers la rive gauche; il $\mathrm{y}$ a donc, à l'entrée du garage aval, une zone d'eau morte favorable à la formation de rouleaux;
TABLEAU V

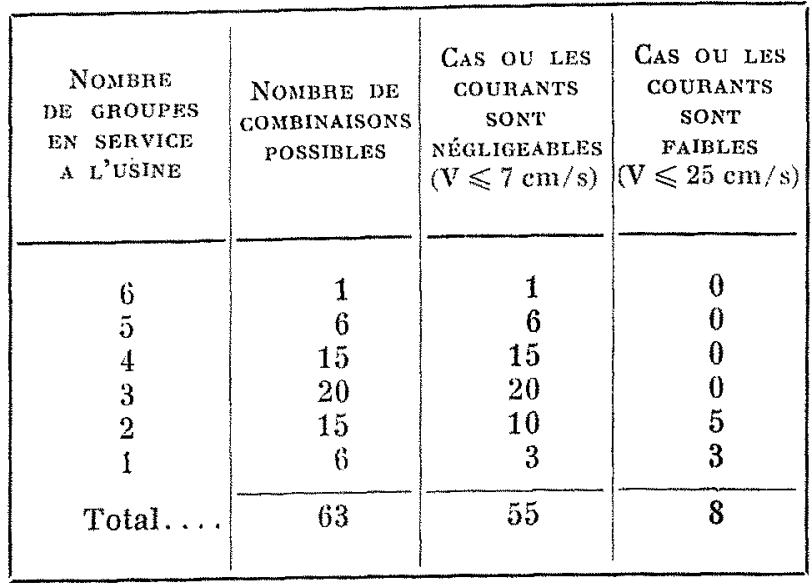

\section{USINE}

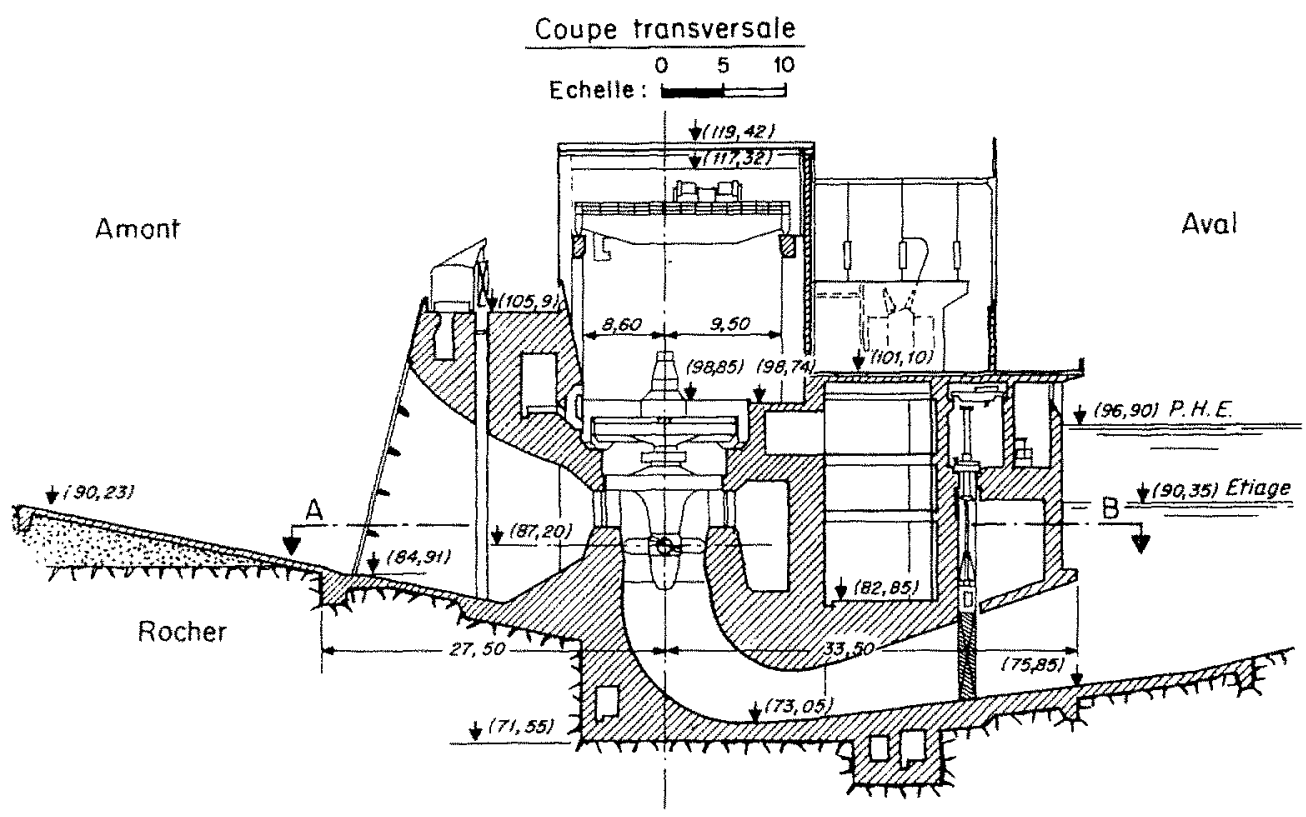

Plan-coupe A-B (sur 1 groupe)

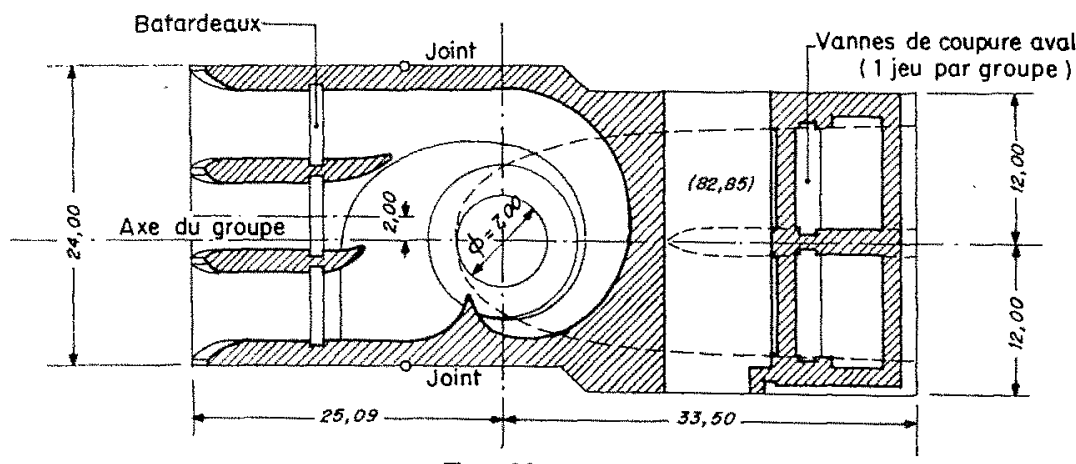

Fig. 39

Coupes transversale et horizontale (au niveau de la bâche spirale) de l'usine de Beauchastel équipée de 6 groupes Kaplan à axe vertical (chacun $34000 \mathrm{kVA} ; 350 \mathrm{~m}^{\mathrm{z}} / \mathrm{s}$, $79 \mathrm{tr} / \mathrm{mn})$. 
- la profondeur du canal étant plus grande qu'au Logis Neuf, l'action de saignées éventuelles creusées dans le fond est nettement plus faible sur l'ensemble des courants.

Les études ont montré que des conditions acceptables pour la navigation ne pouvaient s'obtenir que si un dispositif placé à la sortie des aspirateurs assurait une bonne diffusion des courants sur toute la largeur du canal. La mise au point de ce dispositif a été longue; il se compose en définitive (fig. 40):

- de murs guideaux d'une trentaine de mètres de longueur séparant l'écoulement de chaque groupe; ces guideaux ont, par rapport au sens de l'écoulement, une obliquité de $15^{\circ}$ vers la droite; entre ces guideaux, les radiers se relèvent vers l'aval et se déversent également vers la droite;

- un mur déflecteur est implanté sur la rive gauche, tandis qu'une saignée établie sur l'autre rive dirige vers le centre du canal les courants provenant du déchargeur;

- comme au Logis Neuf, une série de piles circulaires prolonge le mur divisoir.

Enfin, un tapis d'enrochements complète ce dispositif : tout en évitant les affouillements de la marne sous-jacente (fig. 41) il contribue par sa rugosité à la diminution et à la régularisation des vitesses. Ces dispositions (décrochements dans le tracé des radiers, et tapis d'enrochements), qui ont été rendues nécessaires pour les seuls besoins de la navigation, seront les bienvenues lorsque l'écoulement des groupes sera étranglé par les vannes aval, que ce soit pour les besoins d'une coupure d'urgence, ou dans le cas prévu du fonctionnement des groupes en déchargeur (14).

(14) Voici à titre de comparaison les vitesses maximales (en $\mathrm{m} / \mathrm{s}$ ) observées sur le radier bétonné et le plafond du canal de fuite du modèle du Logis Neuf, a une distance $x$ de l'axe des vannes aval de l'usine :

\begin{tabular}{|c|c|c|c|c|c|c|}
\hline & $\begin{array}{l}0 \\
11 \\
0\end{array}$ & $\begin{array}{l}\stackrel{*}{*} \\
\Xi \\
\vec{\Xi} \\
\| \\
\ddot{\ddots}\end{array}$ & 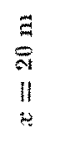 & $\stackrel{\vdots}{9}$ & $\begin{array}{l}\stackrel{*}{*} \\
\stackrel{*}{\Xi} \\
\stackrel{\Xi}{\Xi}\end{array}$ & $\begin{array}{c}\stackrel{E}{a} \\
\stackrel{\Xi}{E} \\
\| 1 \\
0\end{array}$ \\
\hline $\begin{array}{l}\text { Marche, normale (vamne } \\
\text { oxverte, } \mathrm{g} \times 0 \mathrm{pe} \text { en } \\
\text { charge) } \ldots \ldots \ldots \ldots \ldots \ldots\end{array}$ & 6,0 & 4,1 & 3,9 & 3,6 & 3,0 & 2,8 \\
\hline $\begin{array}{l}\text { Controle du debit par } \\
\text { laval (vanne fermée à } \\
90 \% \text { coupure d'urgence } \\
\text { ou marche des groupes } \\
\text { en dechargeur) ......... }\end{array}$ & 15,0 & 11,0 & 8,4 & 5,6 & 3,7 & 2,8 \\
\hline
\end{tabular}

Si les formes du radier aval sont très différentes dans les deux aménagements, les conditions de niveaux et de débits sont pratiquement les mèmes au Logis Neuf et à Beauchastel.

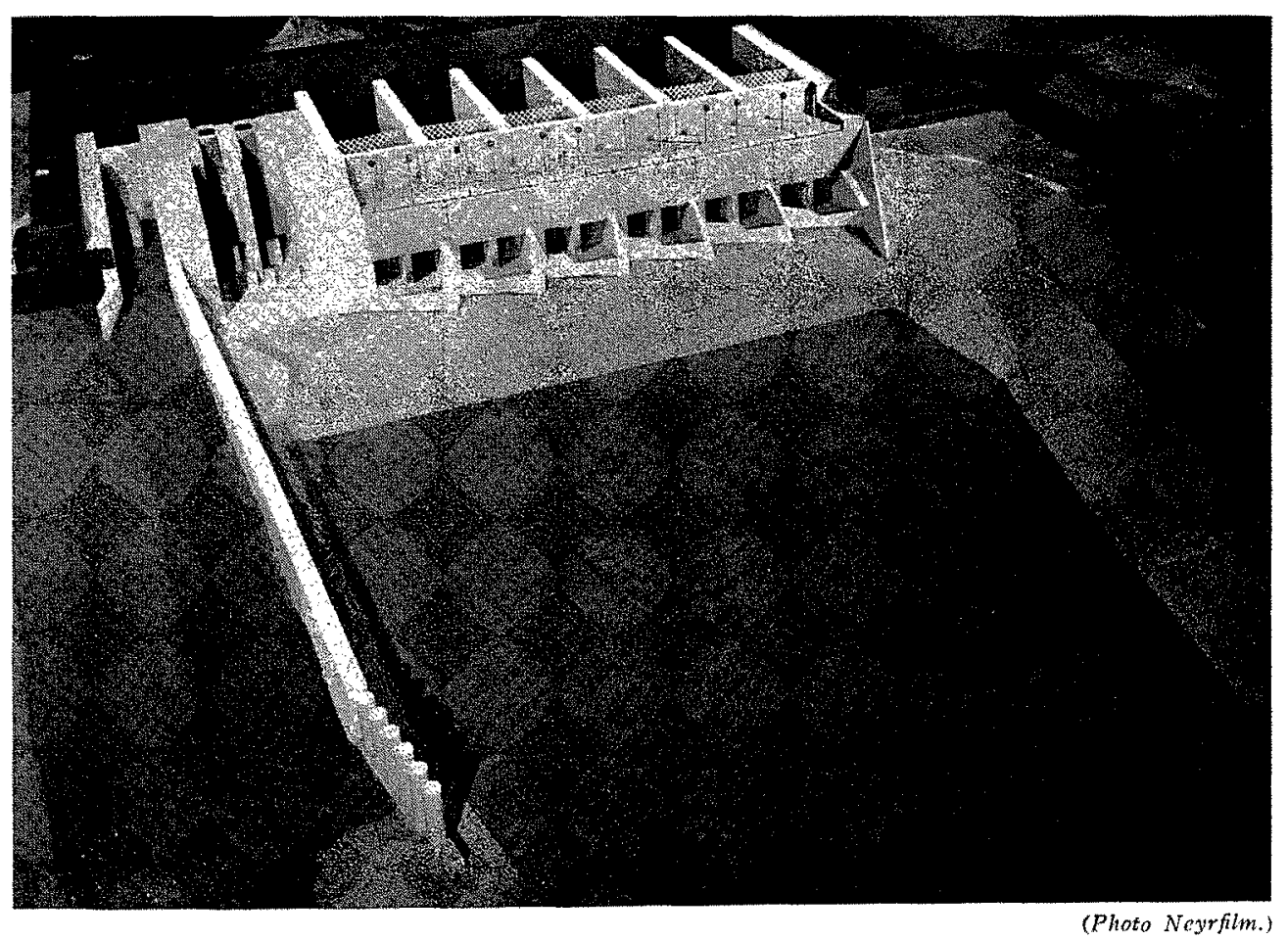

Fra. 40

Vue aval du modẻle représentant l'ensemble usine-déchargeur-écluse de Beauchastel : guideaux et radiérs inclinés, protections en enrochement. 


\subsubsection{ETUdes DES couRANTS FAITES SUR PLACE.}

Les écoulements en aval du bloc usine-déchargeur-écluse, qui ont été étudiés avec beaucoup de soin sur modèle, ont donné lieu à des relevés comparatifs dans la nature grâce à un semis de flotteurs lumineux dont on a photographié les trajectoires de nuit et par temps calme (sans vent) depuis trois observatoires différents. La restitution des photos prises a permis de reconstituer les trajectoires et de vérifier la bonne correspondance du champ des vitesses réelles avec celui déterminé sur modèle. De tels essais, qui ont été effectués il y a quelques années à Donzère, viennent d'être renouvelés tout récemment au Logis-Neuf (fig. 42).

\subsection{Etudes hydrauliques de l'écluse.}

\subsubsection{Alimentation DU Sas.}

En plus d'un bon accès au garage de navigation et au sas de l'écluse, il a été nécessaire d'assurer aux bateaux d'excellentes conditions d'éclusage. Or, l'alimentation et la vidange d'une écluse de grande dimension pourrait, sans précaution, provoquer certains phénomènes hydrauliques gênants :

- Lurbulence dans le sas;

- courants et ondes de translation dans le sas susceptibles d'amorcer des seiches;

- courants dans les garages de navigation.

Pour des surfaces de sas $(195 \times 12 \mathrm{~m})$ et des hauteurs de chute aussi importantes que celles des écluses du Rhône (hauteur maximale de $26 \mathrm{~m}$ à Saint-Pierre, $19 \mathrm{~m}$ à Châteauneuf, 12 à $13 \mathrm{~m}$ pour les autres aménagements), la plupart des solutions classiques ne peuvent s'envisager : alimentation par les têtes, aqueducs latéraux et aqueducs larrons. Nous ne rappellerons pas le détail de l'étude hydraulique de l'écluse de SaintPierre qui a donné lieu à des solutions originales et a fait l'objet de publications diverses $[37$ et 32$]$. Mentionnons simplement les points principaux suivants :

- pour assurer une équirépartition du débit entre les différentes parties du sas, il est nécessaire que les pertes de charge à l'intérieur des différents circuits soient égales entre elles en régime permanent; il en est de même pour les temps de mise en vitesse de ces différents circuits en régime transitoire;

- pour éviter les turbulences, il suffit de ménager un matelas d'eau suffisant et d'introduire l'eau dans le sas par de nombreux per- tuis de section convenable ménages au fond du radier;

- enfin, pour éviler les courants dans les yarages amont et aval, il suffit de prendre l'eau dans le bief usinier amont et de la restituer dans le bief usinier aval. Cette disposition présente en outre l'avantage d'éviter un renouvellement trop fréquent de l'eau des garages et, par suite, de réduire l'importance des envasements. Cette disposition, d'ailleurs très économique, conduit à grouper l'écluse, l'usine et le déchargeur en un bloc unique.

De plus, l'écluse de Saint-Pierre devant être équipée d'une porte intermédiaire située au milieu de sa longueur, on a assuré une alimentation séparée de chaque demi-sas amont et aval, à partir d'un bloc de vannage unique.

Grâce à ces dispositions, la montée du plan d'eau peut s'effectuer sans aucune agitalion gêmante, en un laps de temps très court (7 mn) qui correspond à une vitesse moyenne de montée de $3,4 \mathrm{~m} / \mathrm{mn}$ et une vitesse maximale voisine de $6 \mathrm{~m} / \mathrm{mn}$.

L'éclusage complet peut même c̀tre réalisé avec le dispositif d'alimentation et de vidange d'un seul demi-sas : les vitesses sont alors un peu plus faibles, mais les conditions du sassement restent excellentes (fig. 43).

Ces dispositions, jugées satisfaisantes, ont cté conservées pour l'écluse de Châteanneuf-duRhône, à quelques détails constructifs près. La chute étant un peu plus faible, les sections des aqueducs ont été légèrement réduites pour des raisons d'économie, de manière à obtenir la même durée totale de sassement qu'à SaintPierre.

Aux écluses du Logis Neuf et de Beanchastel, la porte intermédiaire a pu être supprimée. Comme il n'était plus nécessaire d'alimenter séparément les deux demi-sas, on a simplific le système de vannage. Pour conserver une bonne sécurité, les blocs de vannage de remplissage el de vidange, logés cette fois-ci dans les bajoyers, comportent toujours deux séries d'organes en parallèle (vannes, batardeaux, aqueducs). D'autre part, la chute étant notablement plus faible que dans les deux écluses précédentes $(13,50 \mathrm{~m})$, le dispositif d'alimentation a été également simplifié : on a supprimé les chambres intermédiaires de répartition, et les aqueducs secondaires débouchent directement dans le sas par des séries de fentes transversales percées dans le radier de celui-ci. Enfin, on a encore diminué les sections de conduite pour obtenir le même temps total de sassement qu'à SaintPierre. Grâce à ces modifications et à des facilités de fondations, des économies substantielles ont été réalisées sur le coût de l'écluse malgré 
l'accroissement de sécurité de l'équipement (installation de deux portes aval dont l'une est susceptible de couper un débit).

A Pierre-Bénite, la chute est encore un peu plus faible $(11,50 \mathrm{~m})$. D'autre part, les nécessités de fondation ont conduit à loger les aqueducs principaux dans les bajoyers et non dans le radier. Le radier ne contient donc, dans sa partie centrale, que des chambres de répartition alimentées par des aqueducs secondaires. Ceuxci satisfont aux conditions précédemment énoncées d'équirépartition des débits, et débouchent dans le sas par des séries de fentes transver-
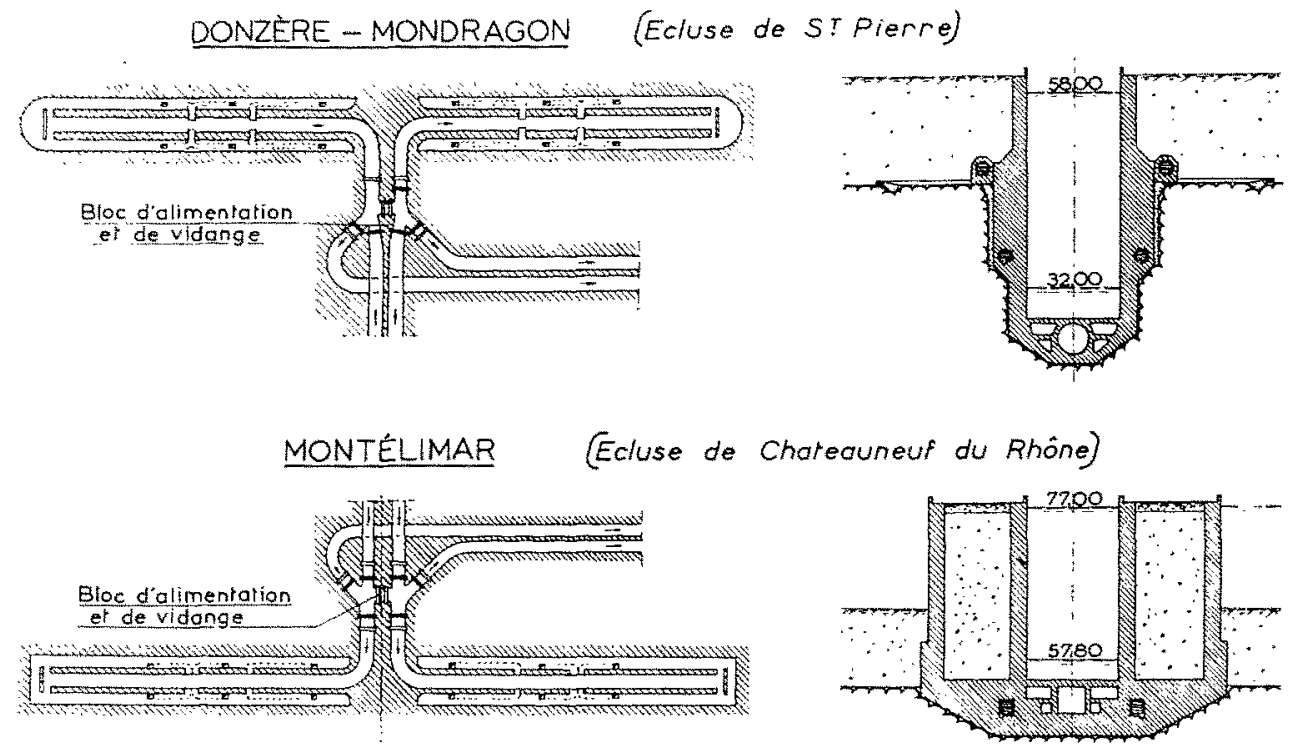

BAIX-LE LOGIS NEUF
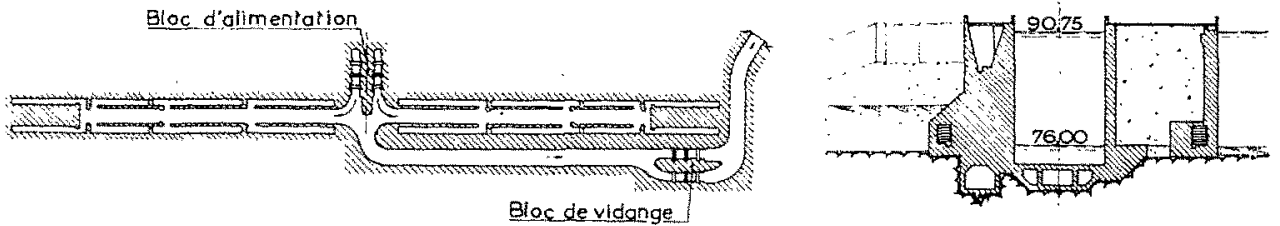

BEAUCHASTEL

Bloc dalimentation
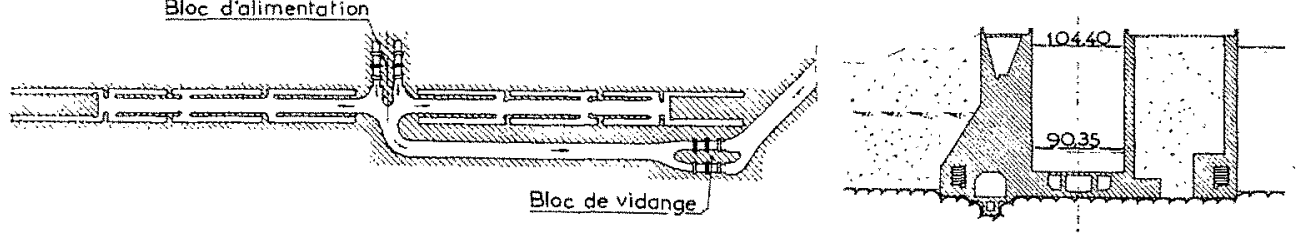

PIERRE - BÉNITE

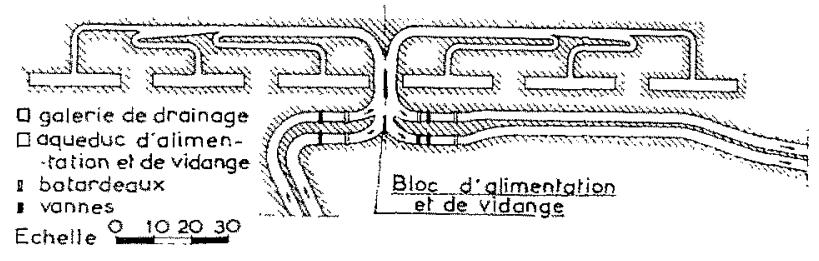

FIG. 43

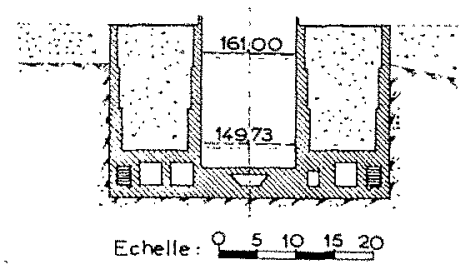

B.E $n \cdot 101$

Schéma des dispositifs dalimentation et de vidange des écluses. 
sales. Quant aux blocs d'alimentation et de vidange, ils ont pu être groupés dans le bajoyer de rive droite, sans augmentation de la largeur de celui-ci.

En résumé, la solution mise au point pour l'écluse de Saint-Pierre a permis de résoudre le problème de l'alimentation et de la vidange de toutes nos écluses de grande surface pour les chutes les plus importantes, avec de grandes vitesses de montée et de descente du plan d'eau, en conservant à l'intérieur du sas une surface très calme ne présentant ni agitation locale, ni ondes ou courants.

Pour les antres chutes plus faibles, de l'ordre de 10 à $14 \mathrm{~m}$, réalisées sur le Rhône, cette solution reste intéressante, en raison notamment des vitesses de montée du plan d'eau qu'elle permet d'obtenir; sa réalisation peut alors recevoir certaines simplifications.

Pour des chutes nettement inférieures à $10 \mathrm{~m}$, la solution perdrait une partie de son intérêt, le temps de montée ou de descente du plan d'eau ne constituant qu'une faible partie du temps total d'éclusage; les solutions d'alimentation par les têtes ou par aqueducs larrons peuvent alors devenir plus intéressantes.

\subsubsection{Chasses a trayers l'écluse.}

Nous avons indiqué que, malgré les précautions prises, des dépôts de limon peuvent se former à l'entrée des garagès amont et aval des écluses. Au surplus, le vent peut y accumuler des corps flottants en période de crue, et des glaces en période de gel, qui gênent l'évolution des bateaux. Dans toutes les écluses, et en particulier à Saint-Pierre et Châteauneuf, on peut sans doute utiliser les circuits hydrauliques alimentant le sas pour créer des chasses. Pour procéder, par exemple, à une chasse dans le garage aval, la porte amont étant fermée et la porte aval ouverte, il suffit d'ouvrir les vannes d'alimentation du sas alors que l'écluse est vide.

De manière à assurer la sécurité complète de ces mancuvres, qui doivent rester exceptionnelles, il faut disposer, sur chaque aqueduc, de deux bouchures en série mancuvrables en charge. Pour des raisons d'économie, on s'est contenté jusqu'à présent de doubler les vannes sur un seul des deux aqueducs de vidange, si bien que l'efficacité des chasses est conditionnée par le débit porté par un seul aqueduc. Ce débit étant relativement modeste (de l'ordre de $70 \mathrm{~m}^{3} / \mathrm{s}$ ), l'efficacité des chasses reste limitée aux abords immédiats des têtes amont et aval des écluses.

A Logis Neuf, Beauchastel et Pierre-Bénite, l'équipement des écluses a été complété par une porte aval manœuvrable en charge; permettant nori seulement un renforcement de l'effet des chasses mais, le cas échéant, la coupure d'un débit passant accidentellement à travers l'écluse. Des essais détaillés sur modèle réduit ont montré les limites d'utilisation de ce procédé. Pour une ouverture partielle de la porte aval intervenant alors que l'écluse est pleine, les vitesses sous le couteau de la vanne pourraient en effet atteindre $18 \mathrm{~m} / \mathrm{s}$. II faut donc localiser le ressaut sur le radier bétonné, et limiter les vitesses à $2 \mathrm{~m} / \mathrm{s}$ dans les parties non revêtues des garages, de manière à éviter l'érosion de berges tout en mobilisant les dépôts.

Moyennant ces limitations, il est possible de faire passer, sans difficultés, à travers les écluses ainsí équipées des débits de l'ordre de 150 à $200 \mathrm{~m}^{3} / \mathrm{s}$. Lors des essais en vraie grandeur exécutés au Logis Neuf, en avril 1961, on s'est limité volontairement à un débit de $130 \mathrm{~m}^{3} / \mathrm{s}$ correspondant à une ouverture de la porte aval de $0,80 \mathrm{~m}$ au-dessus de son seuil, les deux vannes d'alimentation $d u$ sas étant ouvertes. Les conditions générales de l'écoulement se sont révélées absolument conformes aux prévisions du modèle réduit, et l'efficacité des chasses a pu ètre démontrée.

\subsection{Les déchargeurs et les intumescences dans la dérivation.}

Les changements de régime du débit de l'usine provoquent la formation immédiate d'ondes de translation dans les canaux de dérivation. A terme, ils pourraient en outre conduire à un remplissage ou à une vidange exagérée des canaux si la compensation des débits n’était pas assurée.

Les perturbations du plan d'eau sont particulièrement sévères lors des disjonctions électriques, que la situation des lignes de transport rend trop fréquentes, et qui peuvent affecter l'ensemble de l'usine fonctionnant à plein débit. A pleine charge, six groupes Kaplan de l'usine turbinent le débit $q_{e}$ qui est de l'ordre de $2100 \mathrm{~m}^{3} / \mathrm{s}$.

Dès que l'incident se produit, les vannes du distributeur coupent le débit, qui est ramené en l'espace de quelques secondes au débit de marche à vide $v q_{e}$. La disjonction de l'usine se traduit done par la coupure rapide d'un débit $q_{c}=(1-v) q_{e}$ qui est de l'ordre de $0,9 q_{p}$.

Sans précautions spéciales, il pourrait en résulter les inconvénients suivants :

- en amont, le plan d'eau s'éleve et risque de déborder localement ou momentanément pardessus la crête des digues; 
- en aval, le tirant d'eau nécessaire à la navigation risque d'être insuffisant et provoquer le talonnement des bateaux;

- en amont et en aval, la stabilité des digues (15), l'amarrage et la circulation des bateaux risquent d'être affectés par des dénivellations trop importantes ou trop brutales.

Il est donc nécessaire d'étudier de près les répercussions hydrauliques des changements de régime du débit et d'en atténuer, s'il y a lieu, les effets, notamment par un ouvrage déchargeur.

Pour les différentes chutes du bas Rhòne, ces problèmes ont fait l'objet d'études approfondies.

Afin de rétablir le débit dans le canal aval, on dispose, pour les chutes récentes, et notamment au Logis Neuf et à Beauchastel (tabl. VI) :

- pour les effets à court terme, d'un organe à manœuvre automatique rapide: les pertuis de fond du déchargeur qui permettent de réablir environ $0,45 q_{0}$ en l'espace de quelques secondes;

- pour les effets différés, d'organes à manceuvre volontaire et lente (de 10 à $20 \mathrm{mn}$ ) : les pertuis de surface du déchargeur, les circuits de vidange de l'écluse. Il vient s'y ajouter les circuits hydrauliques des groupes de lusine équipés pour la marche en déchargeur automatique. Le débit global qu'il est ainsi possible de rétablir à terme dépasse $0,5 q_{c}$.

Pour les aménagements ultérieurs, dont certains comporteront, à l'instar de l'usine de Pierre-Bénite, des groupes bulbes, qui sont plus maniables que les groupes classiques à axe vertical, et où les pales sont moins sollicitées car les filets liquides ne subissent pas de prérotation avant d'aborder la turbine, nous tenterons de perfectionner encore la marche en déchargeur des groupes (16) de manière à éviter la coupure brutale d'un fort débit lors d'une disjonction. Si un tel perfectionnement paraissait techniquement et ćconomiquement réalisable, on pourrait réduire notablement (si ce n'est supprimer complètement) les effets à court terme que nous venons de signaler. Par voie de conséquence, le déchargeur proprement dit pourrait être encore réduit, voire supprimé.

(15) Ces risques se sont manifestés lors de la mise en eau de l'usine du Logis Neuf. Une disjonction de l'usine a provoqué une onde, qui a été aggravée par une fausse manouvre, alors que le déchargeur n'était pas encore en service: il en est résulté un important glissement dans le talus raide de la digue en gravier séparant en amont les biefs usinier et navigable.

(16) Par exemple par une mancuvre très rapide des organes controlant le debit du groupe : aubes du distributeur, pales, ou vanne aval de sécurité.
Si la totalité du débit ne peut être rétablie très rapidement par le bloc usine-déchargeurecluse, il est indispensable d'utiliser le barrage pour faire transiter le flot par l'intermédiaire du Rhône court-circuité. La capacité utile de la retenue et la tranche utilisable du canal de dérivation sont en effet très faibles devant l'importance du débit d'équipement des usines. Il suffirait d'une coupure complète du débit pendant vingt minutes pour entraîner des débordements.

\subsubsection{Etude hydrauligufe des dêchargeurs.}

L'étude sur modèle réduit des déchargeurs a permis de mettre au point les formes amont et aval des ouvrages de manière à obtenir le meilleur écoulement possible, malgré les vitesses particulièrement élevées $(15 \mathrm{~m} / \mathrm{s})$ qui règnent au droit de la section de contrôle et la quantité importante d'énergie qui se dissipe (plus de $100 \mathrm{MW}$ ).

En amont, on a veillé à une alimentation égale des deux pertuis, malgré la position excentrée du déchargeur, et on a adopté des dispositions spéciales pour limiter les vortex qui tendent à s'établir lorsque les seuls pertuis inférieurs sont ouverts.

En aval, règne une chambre de dissipation de l'énergie, en béton fortement armé, où l'écoulement est très turbulent. Grâce aux obstacles blindés dont elle est garnie, le ressaut se produit dans la partie amont de cette chambre; l'écoulement régulier obtenu à la sortie ne perturbe pas celui des groupes hydroélectriques voisins; il est suffisamment éloigné du régime critique pour éviter des ondulations gênantes en aval. On a étudié la disposition des ouvrages pour éviter des chocs hydrauliques sur la face aval des vannes et on s'est assuré d'une bonne évacuation des corps flottants provenant de l'amont; les formes choisies pour les rainures et les obstacles ont, en outre, permis de limiter la cavitation (fig. 44).

Un des problèmes les plus délicats a consisté à disposer les obstacles en aval des pertuis vannés de façon à obtenir une répartition des vitesses la plus uniforme possible et éviter les courants traversiers. De très nombreuses variantes ont été étudiées en détail sur modèle (plus de quarante pour chaque déchargeur). Le contrôle de la régularisation des vitesses était effectué dans une section, sensiblement rectangulaire, située à l'aval de la chambre de dissipation de l'énergie (à une distance du plan de la vanne de $38 \mathrm{~m}$ à Logis Neuf et $55 \mathrm{~m}$ à Beauchastel). La composante $V_{\text {: }}$ des vitesses dans le sens amont-aval était mesurée au moulinet, d'abord en neuf points distinets (essais de dégrossissage), puis en quinze ou vingt-cinq points 
Tableau VI. -- Conditions de coupure et de rétablissemenl du débit au droil du bloc usine-déchargeur-écluse dans les différentes chutes du Bas-Rhône.

\begin{tabular}{|c|c|c|c|c|c|c|c|c|c|c|c|c|}
\hline \multirow{4}{*}{ DÉSIGNATION DES CHUTES } & \multicolumn{5}{|c|}{ CARACTÉRISTIQUES des GROUPES } & \multicolumn{7}{|c|}{ RÉTABLISSEMENT DU DÉBIT } \\
\hline & \multirow{3}{*}{$\begin{array}{c}\text { DÉBIT } \\
\text { D'ÉQUI- } \\
\text { PEMENT } \\
q_{e} \\
\\
\left(\text { en m }{ }^{8 / s}\right)\end{array}$} & \multirow{3}{*}{$\begin{array}{c}\text { HaUteun } \\
\text { DE } \\
\text { CHUTE } \\
\\
\\
\text { (c) } \mathrm{m})\end{array}$} & \multicolumn{3}{|c|}{ FERMETURE DES GROUPES } & \multicolumn{3}{|c|}{$\begin{array}{l}\text { DÉchaRgEUR : } \\
\text { PERTUIS A MANGEUVRE RAPIDE }\end{array}$} & \multicolumn{4}{|c|}{ Débit rétahli au bout de 5 a $15 \mathrm{mn}$} \\
\hline & & & \multicolumn{2}{|c|}{ Temps de fermeture } & \multirow{2}{*}{$\begin{array}{c}\text { Débit } \\
\text { maximal } \\
\text { coupé } \\
(1-v) \\
(\text { en } \%) \\
\left({ }^{*}\right)\end{array}$} & \multicolumn{2}{|c|}{$\begin{array}{c}\text { Temps } \\
\text { de rétablissement } \\
\text { (cn s) }\end{array}$} & \multirow{2}{*}{$\begin{array}{l}\text { Débit } \\
\text { rétabli } \\
\qquad d_{r} \\
\left.(\text { en } \%)^{*}\right)\end{array}$} & \multirow{2}{*}{$\begin{array}{c}\text { Marche } \\
\text { des } \\
\text { groupes } \\
\text { en dé- } \\
\text { chargeur } \\
g \\
\left(\text { en } \sigma_{t}\right) \\
\left.()^{\circ}\right) \\
\end{array}$} & \multirow{2}{*}{$\begin{array}{c}\text { Déchar- } \\
\text { geur : } \\
\text { pertuis } \\
\text { a manœu- } \\
\text { vre lente } \\
d_{l} \\
(\text { en } \%) \\
(*)\end{array}$} & \multirow{2}{*}{$\begin{array}{c}\text { Ecluse } \\
e \\
e \\
(\text { en } \%) \\
(*)\end{array}$} & \multirow{2}{*}{$\begin{array}{c}\text { Total } \\
g+d_{\iota}+c \\
(\text { en } \%) \\
(*)\end{array}$} \\
\hline & & & $t_{a}$ & $t_{0}$ & & $t_{t}$ & $t_{d}$ & & & & & \\
\hline Donzère-Mondragon. . . . . . . . & 1700 & 24,0 & 3 & 4 & 90 & $\overline{\mathbf{j}}$ & 180 & 90 & - & 9 & 7 & 16 \\
\hline Montélimax.............. & 1920 & 18,5 & 3 & 4 & 90 & 5 & 90 & 47 & -- & 8 & 6 & 14 \\
\hline Baix-Le Logis Neuf......... & 2100 & 13,7 & 3 & 4 & 89 & 2 & 6 & 43 & 33 & 14 & j & 52 \\
\hline Beauchastel. . . . . . . . . . & 2100 & 13,7 & 3 & 4 & 89 & 2 & 6 & 43 & 57 & 10 & 5 & 72 \\
\hline Pierre-Bénite. . . . . . . . . . & 1400 & $10, \tilde{j}$ & 2 & 5 & 89 & 2 & 6 & 35 & 57 & 4 & 7 & 68 \\
\hline
\end{tabular}




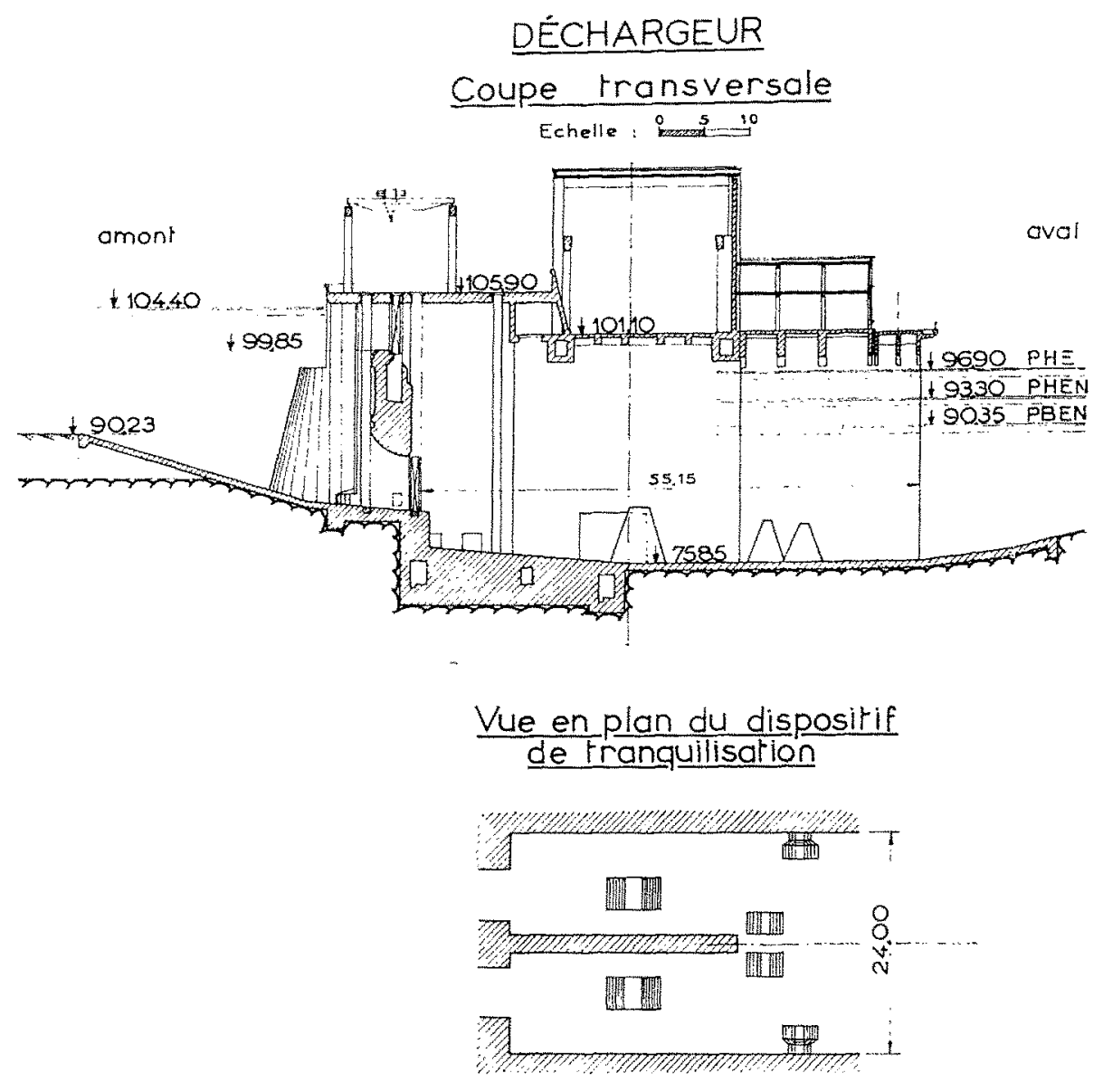

Fxc. 44

Déchargeur de Beauchastel et la chambre de dissipation de l'énergie.

uniformément répartis sur la section (comparaison plus précise des meilleures solutions obtenues).

Pour faciliter la comparaison entre les écoulements obtenus, on a essayé de caractériser par des coefficients chiffrés la valeur de la régularisation obtenue, tant en ce qui concerne l'estimation de la fraction de l'énergie non dissipée que la dissymétrie éventuelle de l'écoulement.

En ce qui concerne le premier point, on s'est ingénié à rendre minimale la dispersion relative $\mathrm{D}$ des vitesses $V_{i}$ autour de la vitesse moyenne :

$$
V_{m}: D=\frac{1}{\sqrt{N}} \sqrt{\sum\left(\frac{V_{i}}{V_{m}}-1\right)^{2}} ;
$$

on a ainsi défini un indice d'uniformité $r=100(1-\mathrm{D})$.

En ce qui concerne la dissymétrie, on a com- paré, pour un écoulement donné, les débits $q_{1}$ et $q_{2}\left(q_{1} \geqslant q_{2}\right)$ qui passent par chacune des deux moitiés de la section de contrôle rectangulaire supposée partagée par une droite passant par le centre. En faisant pivoter la droite de comparaison, la dissymétrie :

$$
\Delta=\frac{q_{1}-q_{2}}{q_{1}+q_{2}}
$$

varie en fonction du coefficient angulaire et passe par un maximum $\Delta m$. Comme précédemment, on a caractérisé la symétrie de l'écoulement par l'indice $\varrho=100(1-\Delta m)$.

Les indices $r$ et ' peuvent, le cas échant, prendre des valeurs négatives, notamment dans le cas d'une très mauvaise distribution des vitesses avec des courants de retour accusés (dispersion et dissymétrie très fortes). Ils peuvent s'annuler, notamment dans le cas où le débit est concentré avec des vitesses uniformes dans une seule 
moitié de la section; les indices atteignent la valeur 100 dans l'hypothèse d'un écoulement absolument uniforme régnant dans toute la section. La figure 45 montre que les résultats obtenus pour les déchargeurs de Baix-Le Logis Neuf et Beauchastel sont excellents. L'indice d'uniformité $r$ est compris entre 73 et 88 et l'indice de symétrie o entre $\$ 5$ et 95 . A titre de comparaison, nous avons fait figurer la distribution des vitesses à la sortie des aspirateurs d'un groupe de Baix-Le Logis Neuf $(r=33 ; 0=48)$.

\subsubsection{LES INTUMESCENCES.}

Les ondes provoquées par les changements de régime de l'usine ont fait l'objet d'études théoriques et d'essais très complets sur modèles réduits (17). De plus, nous avons pu exécuter un certain nombre d'essais en vraie grandeur à

(17) Les fluctuations de niveau dans le temps ont été mesurćes et enregistrées, sur le modèle à $1 / 60$, à l'aide d'un enregistreur graphique d'ondes a quatre roies, qui permettait de comparer simullanément les niveaux en quatre points differents des ouvrages (voir fig. 46 ). Cel appareil est constitué :

- de sondes à capacitance, formées de fil émaillé de très faible diamètre, qui sont plongées dans le courant sans domner lieu à aucun effet parasite de capillarité ou de résistance à l'écoulement;

- d'un bloc électronique détecteur et amplificateur;

- d'un enregistreur composó de galvanographes à très faible inertie se déplaçant devant une même baude enregistreuse.

La rapidité de réponse de eet appareillage permet d'enregistrer aisément, avec un amortissement négligeable, des trains d'ondes dont la période d'oscillation pourrait être inférieure à une demi-seconde (dans la nature).
Fra. 45

Répartition des yitesses à la sortic des déchargeurs et des groupes de l'usine. Mesures effectuées sur les modèles du Logis Neuf et de Beauchastel.

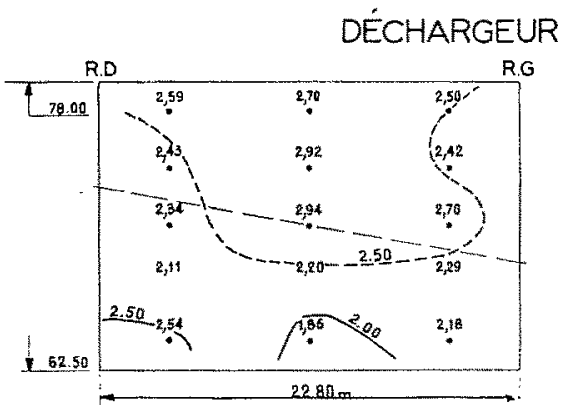

A. Ecoulement ovec 2 pertuis de fond

DU LOGIS NEUF

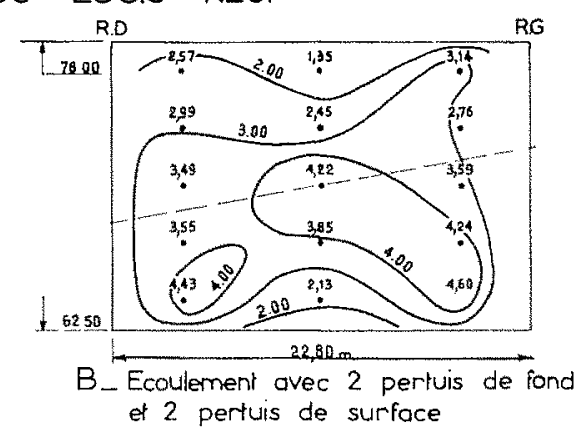

DÉCHARGEUR

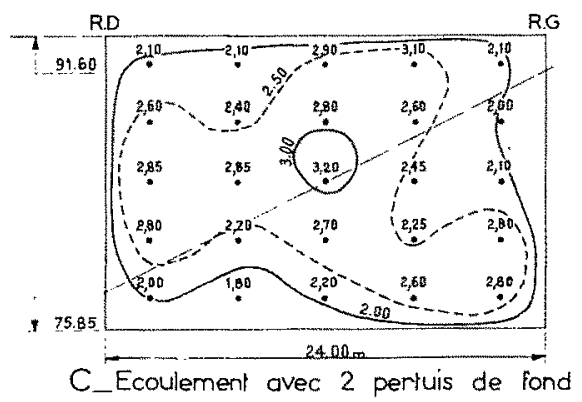

DE BEAUCHASTEL

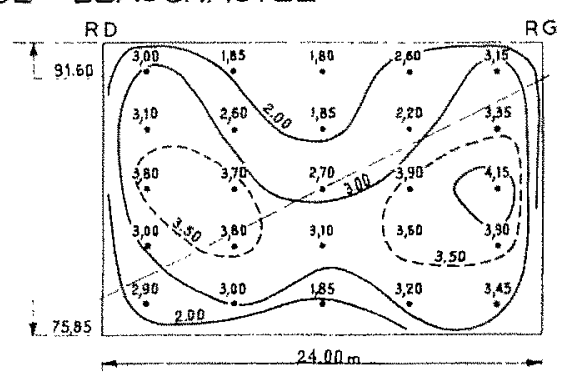

D_Ecoulement avec 2 pertuis de fond el 2 pertuis de surface

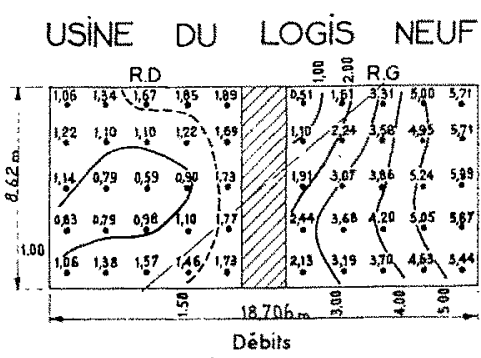

Perluis R.D $=89 \mathrm{~m}^{3} / \mathrm{s} \quad$ Perluis $R S=261 \mathrm{~m}^{3} / \mathrm{s}$

E_Sorlie des aspirateurs

\begin{tabular}{|c|c|c|c|c|}
\hline $\begin{array}{c}\text { ECOULEHEnt } \\
\text { ETHIIi }\end{array}$ & $\underset{\left(\operatorname{sen} \mathrm{m}^{2} / \mathrm{s}\right)}{\operatorname{digit}}$ & $\begin{array}{l}\text { Vitesst } \\
\text { Mothat } \\
{[0 \mathrm{sm} / \mathrm{s})}\end{array}$ & $\begin{array}{c}\text { inoice } \\
\text { outiconnitit } \\
2\end{array}$ & $\begin{array}{c}\text { indict DE } \\
\text { symetgit } \\
\rho\end{array}$ \\
\hline$A$ & 900 & 2.41 & 86 & 93 \\
\hline a & 1.100 & 330 & 73 & 85 \\
\hline c & 900 & 2.50 & 85 & 95 \\
\hline D & 1250 & 302 & 78 & 90 \\
\hline$\varepsilon$ & 350 & 2.58 & 33 & 48 \\
\hline
\end{tabular}

Legender Droite de disymárie maximale 
l'usine du Logis Neuf. C'est dans cette chute que nous choisirons les exemples chifrés qui sont exposés ci-dessous, en insistant spécialement sur les problèmes qui se posent en amont du bloc usine-déchargeur-écluse.

\section{a) Onde initiale en amont de l'usine.}

Rappelons que dans un canal usinier dont les dimensions principales dans la situation iniliale sont : largeur du plan d'eau L, section mouillée $S$, profondeur réduite : $H=(S / L)$, et où règne une vitesse de courant $V_{0}$, la hauteur du corps de l'onde provoquée par la coupure du débit $\Delta q$ est donnée par l'expression :

$$
\mathrm{h}=\frac{\Delta q}{\mathrm{~L}\left(\mathrm{C} \pm \mathrm{V}_{0}\right)} \text {. }
$$

On adopte le signe + si l'onde se propage dans le sens du courant, et le signe - dans le cas contraire.

La célérité $\mathrm{C}$ est donnće par la formule de Boussinesq :

$$
\mathrm{C}=\sqrt{g \mathrm{H}}\left(1+\frac{3}{4} \frac{h}{\mathrm{H}}+\frac{\mathrm{H}^{2}}{6 h} \frac{d^{2} h}{d x^{2}}\right)
$$

Pour les intumescences suffisamment longues, le terme $d^{2} h / d x^{2}$ peut être négligé : la hauteur $h$ de l'onde initiale de translation satisfait à l'équation :

$$
\frac{\Delta q}{\mathrm{~L} h}= \pm \mathrm{V}_{0}+\sqrt{g\left(\mathrm{H}+\frac{3 h}{2}\right)}
$$

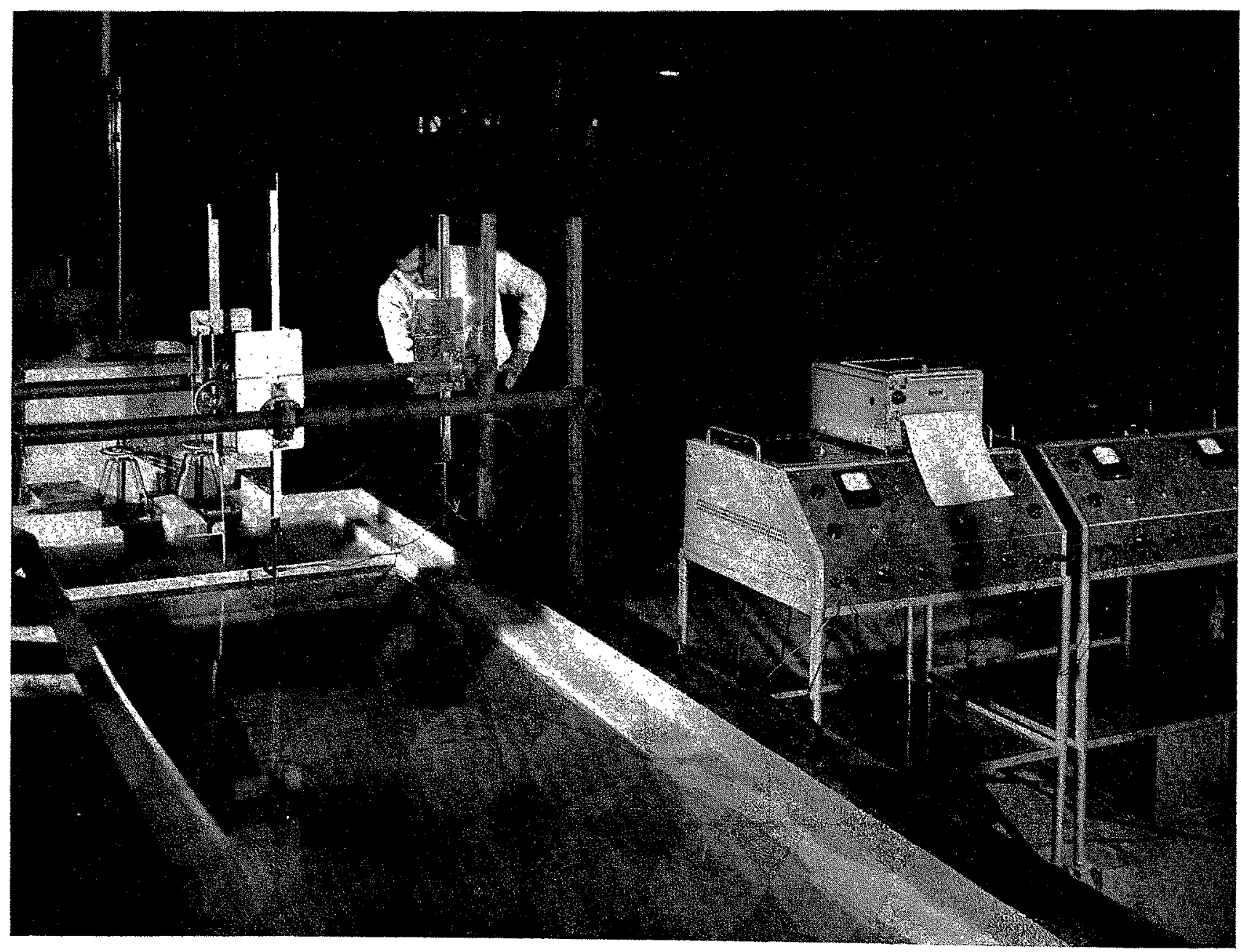

FIa. 46

Etude sur modele des ondes de disjonction. - Dispositif d'enregistrement des ondes. 


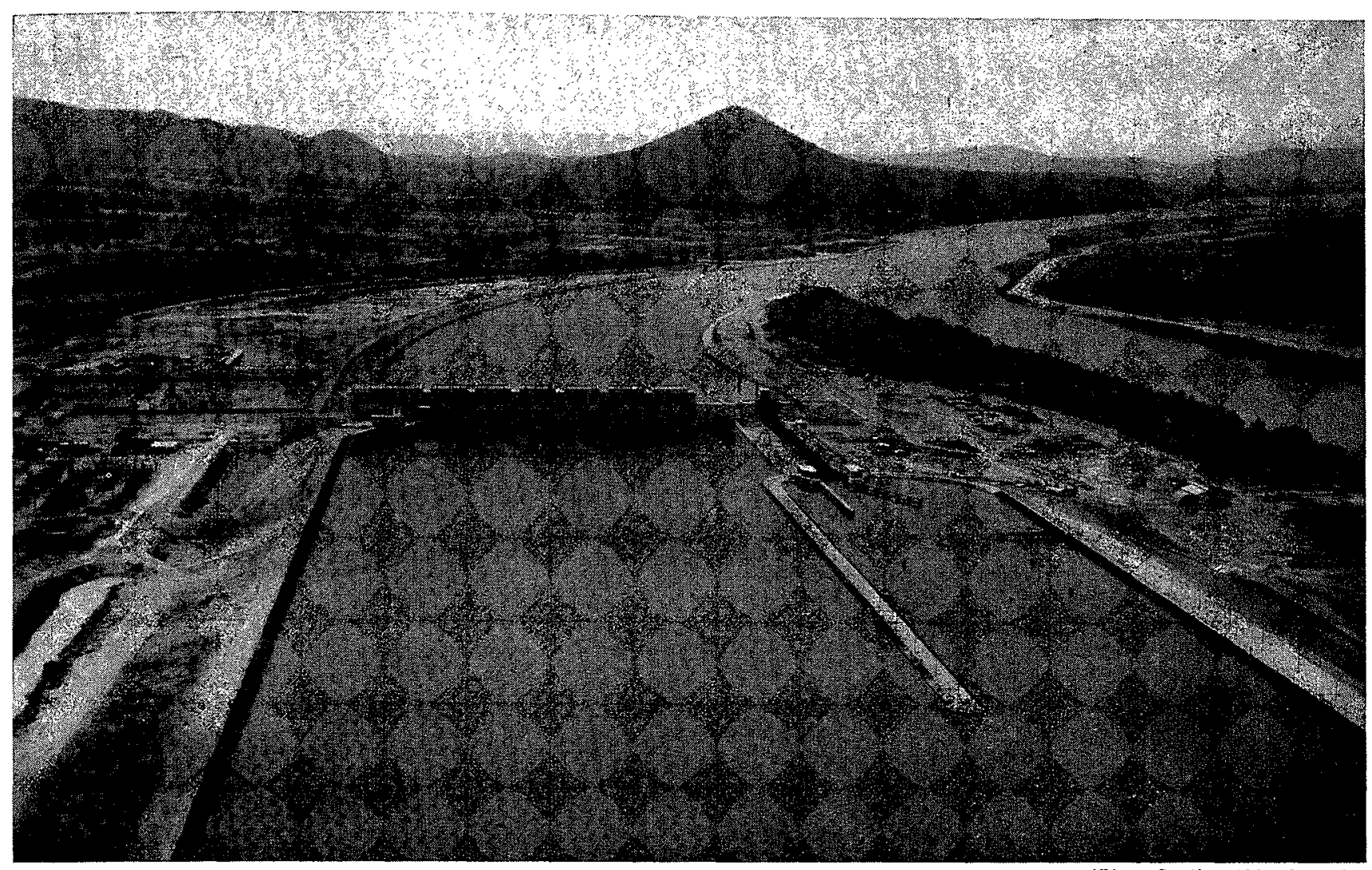

FIG. 47

Vue aérienne amont de l'ensemble des ouvages principaux : usine, déchargeur, écluse de Logis Neuf,

qu'on peut résoudre, en général, plus simplement par approximations successives, qu'en la mettant sous la forme d'une équation du troisième degré en $h$.

En cas de disjonction complète, sans rétablissement de débit par le déchargeur, l'amplitude de l'onde initiale peut être légèrement supérieure à $1 \mathrm{~m}$ à Baix-Le Logis Neuf (fig. 47).

\section{b) Effets secondaires dans le canal usinier :}

A cette onde initiale, se superposent des ondulations du front d'onde de très courte période (15 et $30 \mathrm{~s}$ ). L'une de ces périodes $\left(\mathrm{T}_{1}=15 \mathrm{~s}\right.$ ) coïncide dans le cas présent avec la seiche binodale transversale du canal.

De plus, il y a sur les berges un effet de talus qui renforce encore les ondulations précédentes, l'eau étant projetée sur la berge à un niveau plus élevé que celui de l'onde à une certaine distance de la berge. Par suite de ces amplifications, l'exhaussement maximal de l'eau, notamment sur la berge rive gauche, peut dépasser de beaucoup l'altitude de l'onde théorique et con- duire dans certains cas à une hauteur doublée (18).

(18) H. Favre a montré [31] que la hauteur des ondulations, au-dessus du plan d'cau initial, peut atteindre un maximum de $2,06 h$, lorsque le rapport $h / \mathrm{H}$, de l'onde théorique $h$ sur la profondeur d'eau $\mathrm{H}$, atteint 0,28. En réalité, dans un canal trapézoïdal, il est toujours un point des berges où le rapport $h / \mathrm{H}$ dépasse lá valeur 0,28 . On peut penser que, dans ces conditions, les ondulations risquent d'atteindre, en eet endroit, l'amplitude limite.

D’après les relevés effectuès, soit sur modèle jéduit, soit dans la nature (roir fig. 52 : enregistrement au droit de la Tessonne), il apparaît que les ondulations du front de l'onde ne dépassent pas $1,75 \mathrm{~h}$.

Les enregistrements d'ondes, transversalement au canal, ont confirmé l'observation visuelle de l'amplification sur les berges (voir fig. 48). Leur analyse montre que la longueur d'onde change à proximité de la berge et que des ondulations se produisent de manière systématique en augmentant d'amplitude et de longueur d'onde.

Fig. 48

Amplitude des ondulátions du front de l'onde consécutive à une disjonction et amplification sur les b e r ges (s c h é m a d'après un relevé effectué sur le modèle transversalement au canal).

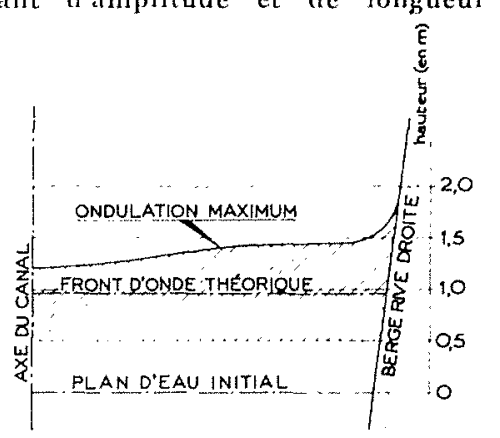




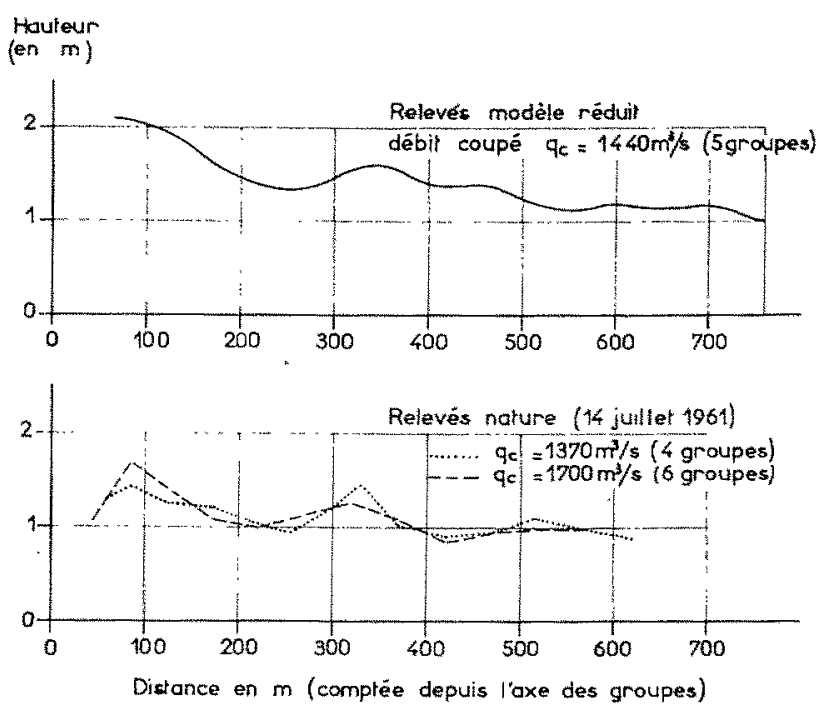

Fra. 49

Graphiques comparatirs des enregistrements dans le canal usinier amont sur modèle et en vraie grandeur (a durée de la coupure clait plus courte sur modèle que dans la nature).

Notamment, en un point particulier situé sur rive gauche à une cinquantaine de mètres du parement de l'usine, la berge présente un changement de direction notable où l'on observe, sur modèle réduit, un exhaussement systématique des niveaux.

Des essais de coupure réalisés à l'usine, notamment en mai 1962, ont montré que la limite extrême de la laisse des eaux a dépassé le niveau initial de $1,50 \mathrm{~m}$ pour un débit coupé $q_{o}=1000 \mathrm{~m}^{3} / \mathrm{s}$ et de $2,10 \mathrm{~m}$ pour :

$q_{c}=1700 \mathrm{~m}^{3} / \mathrm{s}$ (fig. 49 ). On peut estimer qu'en ce point sensible, l'exhaussement serait supérieur à $2,50 \mathrm{~m}$ pour un débit coupé de $2000 \mathrm{~m}^{3} / \mathrm{s}$. Il a donc été nécessaire d'augmenter la revanche des berges dans cette section pour éviter que la digue ne soit submergée accidentellement.

\section{c) Point de branchement des canaux:}

L'onde positive créée dans le canal usinier et agrémentée de ses ondulations secondaires se déplace vers l'amont et atteint au bout d'une minute environ le branchement du garage de navigation amont. La bifurcation se traduit par un élargissement brusque suivi d'un convergent progressif jusqu'à la section courante du canal d'amenée.

Au niveau de cette singularité, l'amplitude de l'onde positive diminue, mais son débit augmente. La différence est compensée par le départ d'une onde négative vers l'aval.

Tableau VII. - Conditions de transmission et de réflexion des intumescences formés dans les canaux en amont de l'usine du Logis Nenf.

\begin{tabular}{|c|c|c|c|c|c|c|c|c|}
\hline & \multirow{3}{*}{$\begin{array}{c}\text { Provmance } \\
\text { DE L'ONDE INCIDENTE }\end{array}$} & \multicolumn{4}{|c|}{$\begin{array}{c}\text { Tnansmission } \\
\text { D'UNE ONDE } \\
\text { INGIDENTE D'AMPLITUdE }+1\end{array}$} & \multirow{3}{*}{$\begin{array}{c}\text { PERTE D'AMPLITUDE } \Delta h / h \\
\text { DANS LE PARCOUHS ALLER } \\
\text { JES CANAUX }\end{array}$} & \multirow{2}{*}{\multicolumn{2}{|c|}{$\begin{array}{l}\text { CONDITIONS DE } \\
\text { RÉFLEXION OU DE } \\
\text { TRANSMISSION D'VNE } \\
\text { ONDE INGIDENTE } \\
\text { (AMPLTUDE + 1) A } \\
\text { L'EXTREMITE } \\
\text { DU CANAL } \\
\text { OPPOSÉE AU } \\
\text { BRANCHEMENT }\end{array}$}} \\
\hline & & \multicolumn{3}{|c|}{$\begin{array}{l}\text { Amplitude } \\
\text { dans les trois canaux } \\
\text { du branchement }\end{array}$} & \multirow{3}{*}{$\begin{array}{c}\text { Retard a la } \\
\text { transmission } \\
\text { au droit du } \\
\text { branchement } \\
(\text { en s) } \\
t_{r} \approx 15\end{array}$} & & & \\
\hline & & $\begin{array}{l}\text { vers } \\
(u i)\end{array}$ & $\begin{array}{l}\text { vers } \\
(n)\end{array}$ & $\begin{array}{l}\text { vers } \\
(a)\end{array}$ & & & $\begin{array}{c}\text { vers le } \\
\text { canal } \\
\text { d'origine }\end{array}$ & $\begin{array}{l}\text { vers la } \\
\text { retenue }\end{array}$ \\
\hline 1 & Canal usinier. (u) & $-0,2$ & $+0,8$ & $+0,8$ & & négligeable (*) & +2 & \\
\hline & 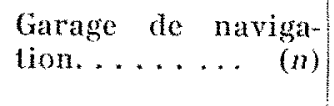 & $+0,32$ & $-0,68$ & $+0,32$ & $t_{r} \approx 15$ & négligeable (*) & +2 & \\
\hline 3 & Canal d'amenée. $(a)$ & $+0,88$ & $+0,88$ & $-0,12$ & $t_{r} \approx 15$ & $\left\{\begin{array}{ccc}14 \% & \text { pour } q_{c}=0,9 q_{e} \\
18 \% & \text { pour } q_{c}=0,5 q_{c} \\
\left({ }^{*}\right)\end{array}\right.$ & $-0,33$ & $+0,67$ \\
\hline & $\begin{array}{l}\text { (*) Pour un } \\
\text { de l'usine jusqua la } \\
\text { th/h est roisin de } \\
\text { (*) Dans le s }^{*} \text { Dan }\end{array}$ & $\begin{array}{l}\text { arcours } \\
\text { porte a } \\
\% \text {. } \\
\text { is aval }\end{array}$ & $\begin{array}{l}\text { de } 2,5 \mathrm{kn} \\
\text { nont de } \\
\text { yers and }\end{array}$ & $\begin{array}{l}\text { corresi } \\
\text { cluse }\end{array}$ & $\begin{array}{l}\text { ndant à un } \\
\text { comportant } \\
\text { ne disiance }\end{array}$ & $\begin{array}{l}\text { allor retour de l'onde d } \\
\text { teux traversées du } \\
\text { de } 7 \mathrm{~km} \text {. }\end{array}$ & $\begin{array}{l}\text { epuis l'a } \\
\text { brancher }\end{array}$ & ont \\
\hline
\end{tabular}


D'une manière générale, en un point de branchement de trois canaux que nous désignerons par le $n^{\circ} 1$ (canal usinier), $n^{\circ} 2$ (garage), $n^{\circ} 3$ (canal d'amenée), si $\mathrm{C}_{i}$ et $\mathrm{L}_{i}$ désignent respectivement la célérité et la largeur du plan d'eau, une onde d'amplitude $h_{0}$ arrivant par l'un des canaux désigné par la lettre $i(i=1,2$ ou 3 ) est transmise dans les deux autres canaux avec une amplitude $\alpha_{i} h_{0}$ tandis que, dans le même canal $n^{\circ} i$, retourne une onde négative d'amplitude $\left(\alpha_{i}-1\right) h_{0}$. $\alpha_{i}$ est donné par la formule :

$$
\alpha_{i}=\frac{2 \mathrm{C}_{i} \mathrm{~L}_{i}}{\Sigma_{1}{ }^{3} \mathrm{C}_{j} \mathrm{~L}_{j}}
$$

Dans le cas du Logis Neuf, on trouve:

$$
\alpha_{1}=0,80 ; \quad \alpha_{2}=0,32 ; \quad \alpha_{3}=0,88 .
$$

L'onde initiale $h_{0}$ se subdivise ainsi (tabl. VII): - le corps principal de l'onde positive remonte le canal mixte d'amenée (amplitude $\left.\alpha_{i} h_{0}=0,8 h_{0}\right)$;

- une onde positive de même amplitude contourne le merlon et entre dans le garage en direction de l'écluse;

- une onde négative de réflexion [amplitude $\left.\left(\alpha_{1}-1\right) h_{0}=-0,2 h_{0}\right]$ revient vers l'usine par le canal usinier.

D'autre part, l'étalement de l'onde par pivotement sur l'extrémité du merlon est suivi d'une réflexion progressive sur les berges de l'entrée du garage: il en résulte une diminution de la raideur du front d'onde initiale, d'où un retard $\left(t_{r}=15 \mathrm{~s}\right)$ dans la transmission complete de l'onde ainsi qu'il apparait sur les enregistrements (fig. 50).

Du point de vue de ses effets, l'onde positive se dirigeant vers l'écluse est la plus importante. En effet, une fois parvenue au fond du garage, elle se réfléchit en conservant son signe et en doublant localement d'amplitude, revient au branchement arec une certaine amplitude $a_{1}$, et $y$ subit une subdivision analogue à celle de la première onde issue de l'usine : e'est-à-dire émission d'ondes positives d'amplitude $\alpha_{2} \alpha_{4}$ ver's les canaux d'amence et usinier et d'une onde négative d'amplitude $\left(x_{2}-1\right) a_{1}$ vers l'écluse, où elle se réfléchit à son tour.

Tout un jeu compliqué de réflexions successives se déroule à chaque passage au droit du branchement avec émission d'ondes positives ou négatives. Seules, les premières phases de ces émissions et réflexions peuvent être observées sur le modèle, par suite des réllexions parasites dues aux limites du modèle qui viennent ensuite perturber les phénomènes. Par contre, une analyse théorique permet de reconstituer tout l'ensemble du systène d'ondes au prix de décomptes un peu fastidieux.

On trouve que, dans les trois canaux, les ondes tendent assez rapidement vers les amplitudes

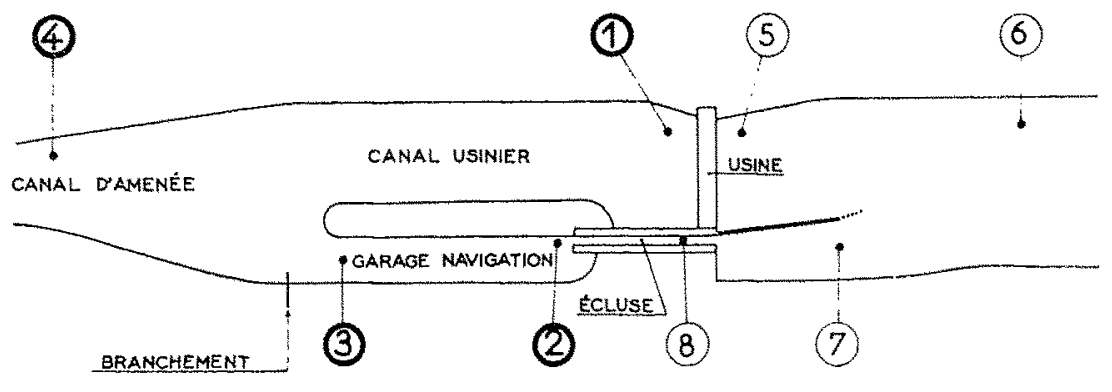

PLAN DE SITUATION DES ENREGISTREMENTS

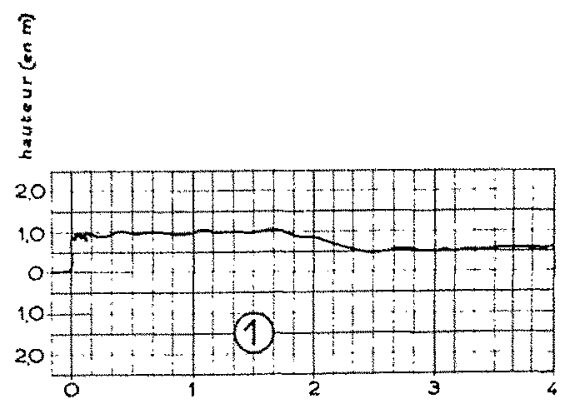

Fig. 50

Enregistrement du départ des ondes consécutives à une disjonction dans les canaux situés en amont de l'usine (modèle du Logis Neuf).

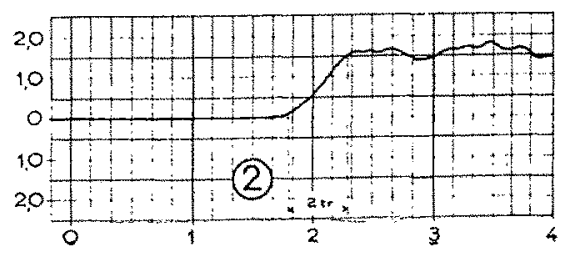

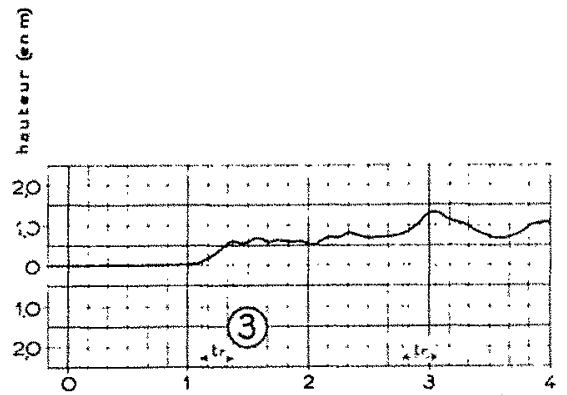

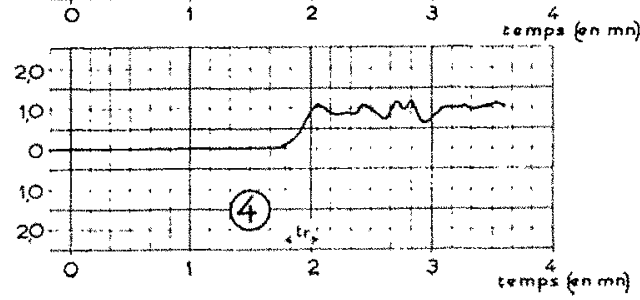


limites $h_{1}, h_{2}$ et $h_{\hat{\mathrm{s}}}$ données par les formules :

$$
\begin{aligned}
& h_{1}=h_{0}\left(1+\frac{2 \alpha_{1}}{\alpha_{3}} \cdot \frac{1-\alpha_{3}}{2-\alpha_{3}}\right)=1,195 h_{0} ; \\
& h_{2}=h_{0} \frac{2 \alpha_{1}}{\alpha_{3}\left(2-\alpha_{3}\right)}=1,63 h_{0} . \\
& h_{3}=h_{0} \frac{\alpha_{1}}{x_{3}}=0,91 h_{0} .
\end{aligned}
$$

d) Seiche longitudinale dans le garage amont et le canal usinier:

Dans tous les cas, le passage d'une onde positive ou négative au droit du branchement donne lieu à une onde d'un certain signe se dirigeant vers l'usine, et à une onde de signe contraire vers l'éciuse.

Il se trouve qu'au Logis Neuf, les temps de parcours dans les deux canaux du branchement (usinier et de navigation) sont sensiblement égaux : $1 \mathrm{mn}$ pour un trajet simple. Il s'ensuit la formation d'une seiche longitudinale (période $\mathrm{T}_{2}=4 \mathrm{mn}$ ) dont les ondes font l'aller et retour entre le parement amont de l'usine et le fond du garage amont.

Le garage de l'écluse constitue ainsi un deuxième point sensible. L'amplitude des ondes initiales $\mathrm{y}$ atteint avant réflexion $0,80 \mathrm{~m}$. Après réflexion, elle peut atteindre $1,70 \mathrm{~m}$ en cas de disjonction totale et non-ouverture du déchar- geur. La revanche des digues et bajoyers de l'écluse doit tenir compte du risque de submersion, qui serait beaucoup plus grave que dans le cas précédent (berge rive gauche de l'usine), car la période des ondes et, par conséquent, la durée d'un déversement éventuel sont ici beaucoup plus grandes $\left[\left(T_{2} / T_{1}\right)=16\right]$.

Ces ondes entretenues de période $\mathrm{T}=4 \mathrm{mn}$ s'amortissent très lentement $(8 \%$ environ à chaque période). Certaines manœuvres ultérieures peuvent les amplifier par effet de résonance, par exemple :

- reprise brutale de la charge d'un ou plusieurs groupes, ou bien ouverture rapide du déchargeur, alors cque le niveau devant l'usine est à son minimum relatif;

- nouvelle disjonction, ou fermeture rapide du déchargeur, alors que le niveau est à un maximum relatif.

C'est ce que l'on a provoqué volontairement, le 14 juillet 1961, lors d'un essai de deux disjonctions successives de $100 \mathrm{MW}$, séparées par un intervalle de $20 \mathrm{mn}$ (soit exactement $5 \mathrm{~T}_{2}$ ). On a constaté que l'amplitude des ondes dans le garage, qui ne dépassait pas $0,70 \mathrm{~m}$ à la première disjonction, a dépassé $1,10 \mathrm{~m}$ à la deuxième (majoration $57 \%$ ), confirmant très exactement les prévisions du calcul (fig. 51).

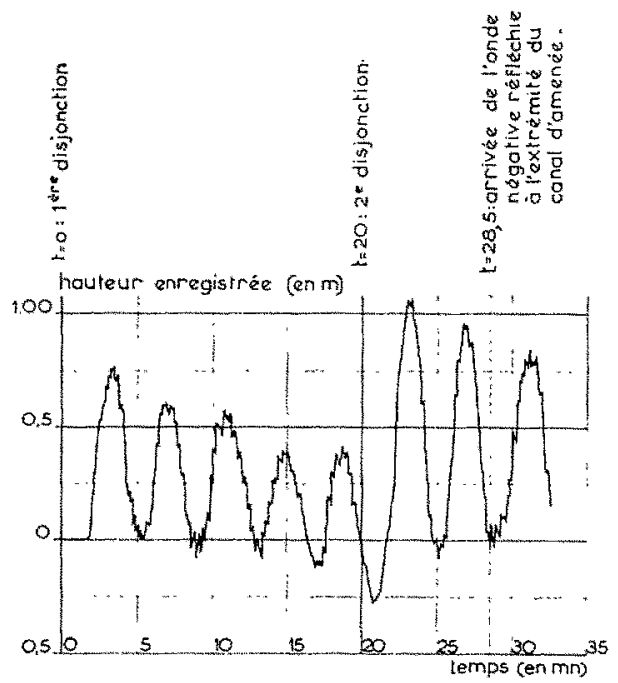

Frg. 51

Comparaison entre les ondes observées dans le garage de navigation du Logis Neuf et celles prévues par le calcul dans des conditions analogues : coupure brutale d'un débit voisin de $1000 \mathrm{~m}^{3} / \mathrm{s}$, rétablissement graduel du débit, nouvelle coupure $20 \mathrm{~mm}$ après la première coupure. Les conditions de l'essai ont différé des hypothèses du caleul sur les points

\begin{tabular}{|c|c|c|}
\hline & $\begin{array}{c}\text { Essat } \\
\text { DU } 14 \text { JUILEET } 1961\end{array}$ & $\begin{array}{c}\text { CALCUL PRÉALABLE } \\
\text { A L'ESSAX }\end{array}$ \\
\hline 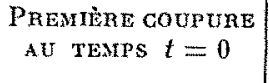 & $\begin{array}{c}q_{0}=900 \mathrm{~m}^{\mathrm{s}} / \mathrm{s} \\
\text { en quelques secondes }\end{array}$ & $\begin{array}{c}q_{c}=1050 \mathrm{~m}^{3} / \mathrm{s} \\
\text { quasi instantanément }\end{array}$ \\
\hline 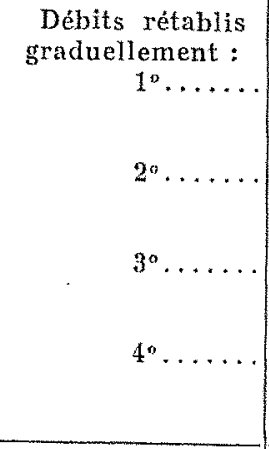 & $\begin{array}{l}\text { Ouverture pertuis RG de- } \\
\text { chargeur entre } t=1 \mathrm{~mm} \\
\text { et } t=6 \mathrm{mn} 15 \mathrm{~s} \text {. } \\
\text { Reprise d'un groupe entre } \\
t=9 \mathrm{mn} 30 \mathrm{~s} \text { et } \\
t=14 \mathrm{mn} \text {. } \\
\text { Reprise d'un groupe entre } \\
t=14 \mathrm{mn} 5 \mathrm{~s} \text { et } \\
t=18 \mathrm{~mm} 30 \mathrm{~s} \\
\text { Fermeture déchargeur } \\
\text { entre } t=15 \mathrm{mn} 30 \mathrm{~s} \text { et } \\
t=20 \mathrm{mn} \text {. }\end{array}$ & $\begin{array}{l}\text { Reprise d'un groupe entre } \\
t=5 \mathrm{mn} 45 \mathrm{~s} \text { et } \\
t=8 \mathrm{mn} 45 \mathrm{~s} \text {. } \\
\text { Reprise d'un groupe entre } \\
t=9 \mathrm{mn} 30 \mathrm{~s} \text { et } \\
t=15 \mathrm{mn} \text {. } \\
\text { Reprise d'un groupe entre } \\
t=17 \mathrm{mn} \text { et } t=20 \mathrm{mn} \text {. }\end{array}$ \\
\hline $\begin{array}{cc}\text { Nouvelle } & \text { cou- } \\
\text { pure au } & \text { temps } \\
t=20 \mathrm{mn} & \ldots\end{array}$ & $q_{0}=900 \mathrm{~m}^{3} / \mathrm{s}$ & $q_{c}=1050 \mathrm{~m}^{3} / \mathrm{s}$ \\
\hline
\end{tabular}
suivants :

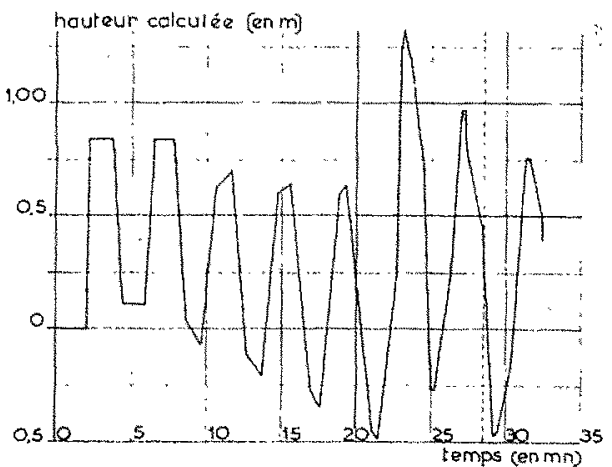


Pour éviter ce danger de résonance, les consignes d'exploitation prévoient une reprise en charges des groupes d'une manière lente et la plus progressive possible (maximum $10 \mathrm{MW}$ par minute).

Après un déclenchement très important ayant occasionné des ondes de période $4 \mathrm{mn}$ et de très forte amplitude, il y a lieu d'attendre l'amortissement de ces ondes avant de reprendre la charge, de manière à ne pas risquer un deuxième déclenchement intéressant à nouveau un débit voisin du maximum.

Pour éviter les fausses manœuvres et rendre pratique l'application de cette consigne, sans trop gêner l'exploitation, nous avons prévu de transmettre systématiquement à la salle de commande de l'usine l'enregistrement des ondes devant l'écluse.

\section{e) Canal d'amenée et retenue :}

Enfin, l'ondè principale qui s'est engagée dans le canal continue sa progression. Elle reste agrémentée d'ondulations secondaires qui sont
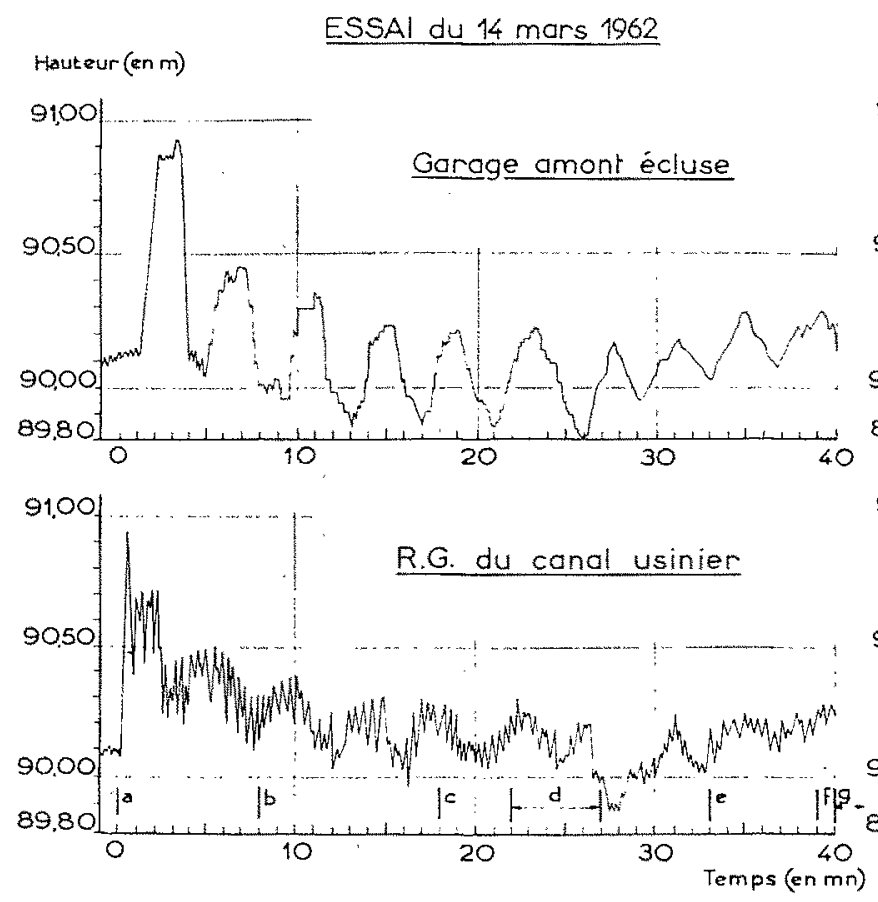

très importantes (fig. 52) comme nous avons pu le constater par un limnigraphe installé vers la Tessonne (à $2 \mathrm{~km}$ de l'usine).

Elle subit, pendant son parcours, une perte d'amplitude qui est relativement faible (comme les pertes de charge dans le canal dont elle dépend). Lorsque cette onde atteint l'origine du canal avec une certaine amplitude $a_{s}$, les caractéristiques de la prise (notamment L et C) sont telles qu'une partie de l'onde continue son chemin (avec l'amplitude $0,67 a_{2}$ ) tandis qu'elle renvoie dans le canal une onde négative d'amplitude $-0,33 \alpha_{2}$.

Cette onde revient à l'usine environ 27 à $29 \mathrm{mn}$ après la disjonction, et dans certains cas nous avons pu enregistrer plusieurs allers et retours d'une seiche de période $T_{3}=28 \mathrm{mn}$ environ, intéressant toute la longueur du canal.

L'onde principale remontant la retenue est encore observable vers La Voulte (à $15 \mathrm{~km}$ de l'usine), mais, que ce soit dans le canal d'amenée ou dans la retenue, les dénivellations instantanées ne paraissent pas particulièremënt dangereuses.

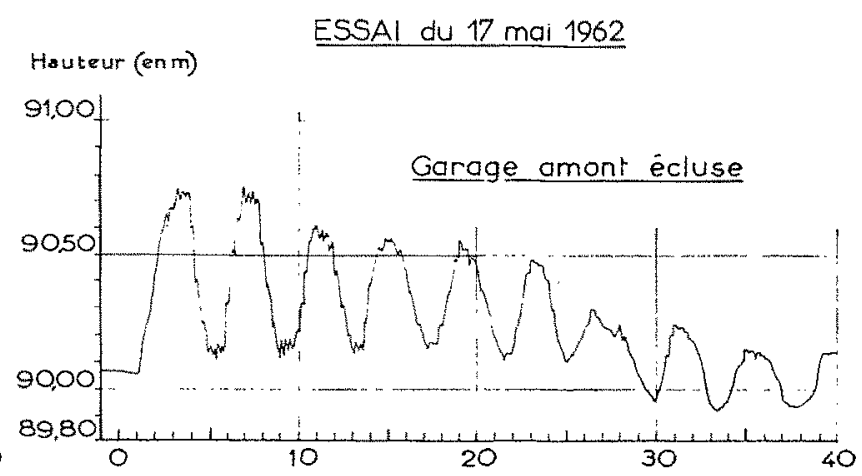

\section{LEGENDE}

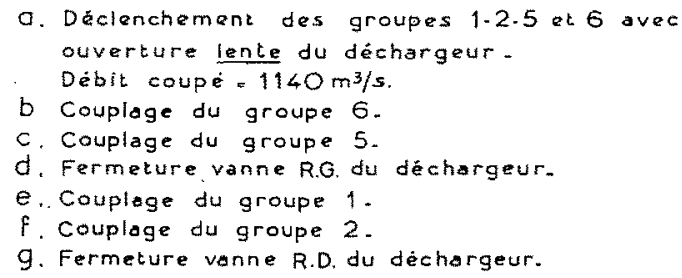

F1G. 52

Essais de coupure réalisés à l'usine du Logis Neuf les 14 mars 1962 et 17 mai 1962. Enregistrement dans les canaux en amont du bloc usine-déchargeur-écluse. 


\section{f) Intervention du déchargeur:}

Lorsque le dechargeur n'intervient pas, l'onde initiale $h$ devant l'usine dépend du débit coupé $q_{t}=(1-b) q_{e}$. En amont, devant les diverses branches du canal, les dénivellations et les ondulations ne dépendent que de la valeur $h$ initiale. Nous avons vu qu'clles peuvent être importantes et provoquer une gêne assez sérieuse.

Lorsque le déchargeur intervient rapidement pour rétablir un débit $q_{d}$, l'amplitude de l'onde initiale au droit de l'usine n'est pas la même sur rive droite (devant le déchargeur) que sur rive gauche (où le déchargeur ne fait sentir son action qu'avec un certain retard, en raison de la largeur du canal).

Sur rive gauche, l'amplitude initiale est, comme dans le cas précédent, $h$ qui dépend de $q_{i}$ et non de $q_{1} ;$ sur l'autre rive, l'amplitude initiale $k_{0}$ est donnée par l'expression suivante :

$$
k_{0}=h\left[1-\frac{q_{d}}{q_{0}} \frac{t_{0}+\left(t_{0}-t_{1}\right)}{t_{a}}\right]
$$

qui fait intervenir non seulement les débits $q_{i}$ et $q_{c}$, mais aussi quatre constantes de temps. La mancuvre des groupes et du déchargeur n'est en effet pas instantanée; aux temps morts initiaux comptés à partir de la disjonction électrique $\left(t_{0}=3 \mathrm{~s}\right.$ pour les groupes, $t_{1}=2 \mathrm{~s}$ pour le déchargeur), s'ajoutent respectivement la durée pendant laquelle la variation du débit s'effectue de manière sensiblement linéaire $\left(t_{e}\right.$ pour la coupure du débit $q_{0}, t_{t}$ pour le rétablissement du débit $q_{i}$ ).

Dans les diverses branches de l'amont, l'onde positive énise par l'usine (coupure de $\eta_{c}$ ) est rattrapée par l'onde négative ćmise par le déchargeur (rétablissement de $q_{d}$ ) si $t_{1}-t_{0}$ n'est pas trop grand. L'estimation de la hauteur des ondes fait alors intervenir le retard $t_{r}$ subi par les ondes au branchement dont il a été question plus haut, si bien que la hauteur dépend non seulement de $k_{0}$, mais de la série des valeurs $k_{3 k}$ :

$$
k_{n}=n\left(1-\frac{q_{d}}{q_{c}} \frac{t_{b}+\left(t_{n}-t_{1}\right)+n t_{r^{2}}}{t_{d}+n t_{r}}\right)
$$

$n$ étant le nombre de passages au branchement marquant l'entrée du garage.

Le tableau VIII récapitulatif indique les valeurs prises on amont de l'usine par le corps de l'onde principale, par les ondulations du front de l'onde ef réflexions transversales, en fonction des amplitudes initiales $h$ el $k_{0}$ devant lusine, et de la suite des amplitudes $k_{n}$ ci-dessus définies.

Un abaque (fig. 53) montre l'efficacité du dechargeur du Logis Neuf (exprimée par le coefficient d'afténuation des amplitudes $h / k_{0}$ ) pour les diverses valeurs des constantes de temps $\left(t_{1}\right.$ et $t_{d}$ du déchargenr. On a admis ici un débit coupé $q_{0}=1900 \mathrm{~m}^{*} / \mathrm{s}$, un débit rétabli par le

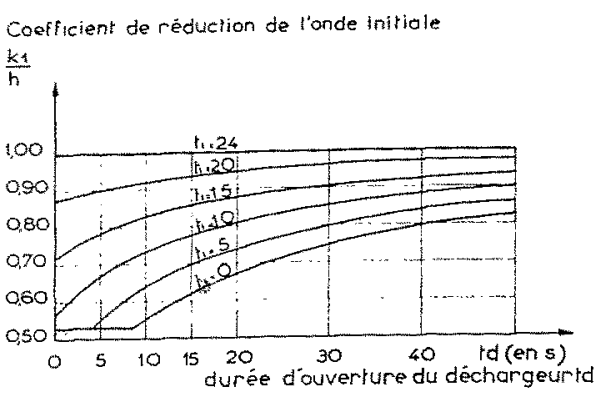

ti..retord de louverture du dechargeur (en s)

FiG. 53

Influence de la loi d'ouverture du déchargeur sur l'amplitude des ondes dans le garage amont de l'écluse du Logis Neuf. Les parametres sont définis comme suit : $t_{1}=$ retard de l'ouverture des vannes du dechargeur; $t_{i z}=$ durée de l'ouverture des vannes du déchargeur;

$l_{i l}=$ amplitude de la premiere onde devant la porte de l'écluse et dans le canal d'amenée avec intervention du déchargeur;

$h=$ amplitude de la première onde sans intervention du déchargeur.

déchargeur $q_{i t}=900 \mathrm{~m}^{3} / \mathrm{s} ;$ les constantes de temps des groupes sont $t_{0}=3 \mathrm{~s}, t_{c}=4 \mathrm{~s}$.

Pour que le déchargeur ait une efficacité voisine de son maximum $(0,55)$, les constantes de temps qui expriment en secondes la rapidité de la manouvre, doivent satisfaire à l'inégalité $t_{l l}+0,72 t_{1} \leqslant 10$, ce qui suppose par conséquent, un fonctionnement presque immédiat. Si le premier terme de l'inégalité atteint $20 \mathrm{~s}$ au lieu de $10 \mathrm{~s}$, le coefficient de réduction des ondes n'est que de 0,68 .

Nous avons pu contrôler expérimentalement: des résultats dérivant du calcul, en faisant varier réellement le temps de mise en service du dechargeur. Au Logis Neuf, l'ouverture des vannes est réglée par un automate installé sur. le wattmètre totalisateur de l'usine. Cet automate commande l'ouverture rapide d'un ou deux pertuis du déchargeur, suivant que la réduction. de puissance instantanée est comprise entre 60 et $120 \mathrm{MW}$ ou dépasse $120 \mathrm{MW}$.

Il est muni d'une mémoire temporaire qui s'efface à une vitesse de l'ordre de $1 \mathrm{MW}$ par. seconde afin d'ćviter les ouvertures intempestives du déchargeur.

L'efficacité de l'action du déchargeur a été mise en évidence par les résultats suivants (fig. 52) :

- le 14 mars 1962, avec un débit coupé de $1140 \mathrm{~m}^{3} / \mathrm{s}$ et une ouverture lente du déchargeur, l'amplitude des ondes dans le garage de l'écluse a été de $0,80 \mathrm{~m}$;

- le 17 mai 1962, pour un débit coupé ¿e. $1700 \mathrm{~m}^{3} / \mathrm{s}$ suivi d'une ouverture rapide des deux pertuis du déchargeur commandée par l'automate, cette même amplitude n'a été que de $0,68 \mathrm{~m}$; sans intervention du déchargeur, elle aurait atteint dans ce cas $1,20 \mathrm{~m}$ environ. 
Tableau récapitulatif des calculs d'ondes dues à une disjonction en amont et en aval de l'usine du Logis Nenf (avec ou sans mise en service du déchargeur à ouverture rapide.)

\section{EN AMONT DE L'USINE}

\begin{tabular}{|c|c|c|c|c|c|c|c|c|c|c|c|c|c|c|}
\hline \multirow{3}{*}{\multicolumn{2}{|c|}{ Nature de L'iNTUMEScence }} & \multicolumn{5}{|c|}{ CANal USINIER AMONT } & \multicolumn{5}{|c|}{ GaRAGE AMONT NAVIGATION } & \multicolumn{2}{|c|}{ Canal amenée } & \multirow{3}{*}{$\begin{array}{l}\text { RETENuE } \\
\text { AMONT } \\
\text { au-delá } \\
\text { de } 13 \mathrm{mn}\end{array}$} \\
\hline & & \multicolumn{2}{|c|}{0 a $2 \mathrm{mn}$} & \multirow{2}{*}{$2 \mathrm{a} 4 \mathrm{mn}$} & \multirow{2}{*}{4 à $6 \mathrm{mn}$} & \multirow{2}{*}{6 a $8 \mathrm{mn}$} & \multirow{2}{*}{$1 \mathrm{a} 2 \mathrm{mn}$} & \multirow{2}{*}{2 a $3 \mathrm{mn}$} & \multirow{2}{*}{3 à $5 \mathrm{mn}$} & \multirow{2}{*}{5 à $7 \mathrm{mn}$} & \multirow{2}{*}{7 à $9 \mathrm{mn}$} & \multirow{2}{*}{1 a $3 \mathrm{mn}$} & \multirow{2}{*}{$\begin{array}{c}\text { au-delà } \\
\text { de } 3 \mathrm{mn}\end{array}$} & \\
\hline & & R.G. & R.D. & & & & & & & & & & & \\
\hline \multirow{3}{*}{ 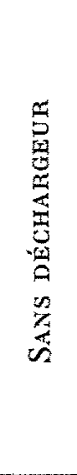 } & $\begin{array}{c}\text { Onde principale ou } \\
\text { corps de l'onde... }\end{array}$ & $+h$ & $+h$ & $+0,6 h$ & $+1,2 \mathrm{~h}$ & $+0,6 h$ & $+0,8 h$ & $+1,6 h$ & $+0,2 h$ & $+1,6 h$ & $+0,2 h$ & $+0,8 h$ & $+0,9 h$ & $+0,6 h$ \\
\hline & $\begin{array}{c}\text { Ondulation du front } \\
\text { et, ou, réffexions } \\
\text { transversales (pé- } \\
\text { riode } 15 \text { et } 30 \mathrm{~s} \text { ). }\end{array}$ & $\begin{array}{l} \\
+0,4 h \text { à } \\
+1,4 h \\
\text { localement }\end{array}$ & $\pm 0,4 h$ & $\pm 0,4 h$ & $\pm 0,4 h$ & $\pm 0,4 h$ & $\pm 0,1 h$ & $\pm 0,1 h$ & $\pm 0,1 \mathrm{~h}$ & $\pm 0,1 \mathrm{~h}$ & $\pm 0,1 h$ & $\pm 0,6 \mathrm{~h}$ & $\pm 0,6 \mathrm{~h}$ & \\
\hline & $\begin{array}{ll} & \operatorname{maxi} \ldots \\
\text { Total }: & \\
& \operatorname{mini} . \\
\end{array}$ & $\begin{array}{c}+1,4 h \text { à } \\
+2,4 h \\
-\end{array}$ & $\begin{array}{l}+1,4 h \\
+0,6 h\end{array}$ & $\begin{array}{l}+1 h \\
+0,2 h\end{array}$ & $\begin{array}{l}+1,6 h \\
+0,6 h\end{array}$ & $\begin{array}{l}+1 h \\
+0,2 h \\
\end{array}$ & $\begin{array}{r}+0,9 h \\
+0,7 h \\
\end{array}$ & $\begin{array}{l}+1,7 h \\
+1,5 h\end{array}$ & $\begin{array}{l}+0,3 h \\
+0,1 h\end{array}$ & $\begin{array}{l}+1,7 \mathrm{~h} \\
+1, \tilde{b} \mathrm{~h}\end{array}$ & $\begin{array}{l}+0,3 h \\
+0,1 h \\
\end{array}$ & $\begin{array}{l}+1,4 h \\
+0,2 h\end{array}$ & $\begin{array}{l}+1,5 h \\
+0,3 h \\
\end{array}$ & $+0,6 \mathrm{~h}$ \\
\hline \multirow{3}{*}{ 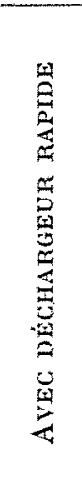 } & $\begin{array}{c}\text { Onde principale ou } \\
\text { corps de l'onde... }\end{array}$ & $+h$ & $+k_{0}$ & $+0,6 k_{1}$ & $+1,2 k_{2}$ & $+0,6 k_{3}$ & $+0,8 k_{1}$ & $+1,6 k_{1}$ & $+0,2 k_{2}$ & $+1,6 k_{3}$ & $+0,2 k_{4}$ & $+0,8 k_{1}$ & $+0,9 k_{3_{2}}$ & $+0,6 k_{2}$ \\
\hline & $\begin{array}{l}\text { Ondulation du front } \\
\text { et, ou, réflexions } \\
\text { transversales (pé- } \\
\text { riode } 15 \text { et } 30 \mathrm{~s} \text { ).. }\end{array}$ & $\begin{array}{l} \\
+0,4 h \dot{a} \\
+1,4 h \\
\text { localement }\end{array}$ & $\pm 0,4 h$ & $\pm 0,4 h$ & $\pm 0,4 \mathrm{k}_{2}$ & $\pm 0,4 k_{3}$ & $\pm 0,1 k_{1}$ & $\pm 0,1 k_{1}$ & $\pm 0,1 k_{2}$ & $\pm 0,1 k_{3}$ & $\pm 0,1 k_{4}$ & $\pm 0,6 k_{1}$ & $\pm 0,6 k_{2}$ & \\
\hline & Total: & $\begin{array}{l}+1,4 h \mathrm{a} \\
+2,4 h \\
-\end{array}$ & $\begin{array}{l}k_{0}+0,4 h \\
k_{0}-0,4 h\end{array}$ & $\left|\begin{array}{c}+0,6 k_{1} \\
+0,4 h \\
0,6 k_{1} \\
-0,4 h\end{array}\right|$ & $\begin{array}{l}+1,6 k_{2} \\
+0,8 k_{3}\end{array}$ & $\begin{array}{l}+k_{3} \\
+0,2 k_{3}\end{array} \mid$ & $\begin{array}{l}+0,9 k_{1} \\
+0,7 k_{1}\end{array}$ & $\begin{array}{l}+1,7 k_{1} \\
+1,5 k_{1}\end{array}$ & $\begin{array}{l}+0,3 k_{2} \\
+0,1 k_{2}\end{array} \mid$ & $\left|\begin{array}{l}+1,7 k_{3} \\
+1,5 k_{3}\end{array}\right|$ & $\begin{array}{l}+0,3 k_{4} \\
+0,1 k_{4}\end{array}$ & $\begin{array}{l}+1,4 k_{1} \\
+0,2 k_{1}\end{array}$ & $\begin{array}{l}+1,5 k_{z} \\
+0,3 k_{2}\end{array}$ & $+0,6 k$ \\
\hline \multicolumn{15}{|c|}{$\begin{array}{l}h \text { désigne ici la hauteur de l'onde théorique produite dan } \\
\text { vention du déchargeur). } \\
k_{t:} \text { désigne la hauteur de l'onde résultante dans le canal us } \\
\left.\text { déchargeur rapide rétablissant le débit } q_{d}\right): k_{n}:=h[1 \\
k^{\prime} \text { hauteurs homologues en aval de l'usine. }\end{array}$} \\
\hline
\end{tabular}


Tableau VIII (suite).

EN AVAL, DE L'USINE

\begin{tabular}{|c|c|c|c|}
\hline \multicolumn{2}{|c|}{ Situation } & SANS DÉCHARGEUn & AVEC DECKARGEUR \\
\hline \multirow{2}{*}{ Canal usinier................ } & Première onde 0 à $1 \mathrm{mn} \ldots \ldots \ldots$ & $-h^{\prime}$ & $-h^{\prime}$ \\
\hline & Deuxième onde 2 à 3 mn........ & $-1,2 h^{\prime}$ & $-1,2 k^{\prime}$ \\
\hline Garage aval............... & $1 \dot{a} 2 \operatorname{mn} \ldots \ldots \ldots \ldots \ldots \ldots \ldots$ & $-1,5 h^{\prime}$ & $-1,5 k^{\prime}$ \\
\hline \multirow{2}{*}{ Canal de fuite................ } & Première onde 0,5 à $1,5 \mathrm{mn} \ldots \ldots$ & $-0,75 h^{\prime}$ & \multirow{2}{*}{$-k^{\prime}$} \\
\hline & Deuxième onde au-delà de $1,5 \mathrm{mn}$. & $-h^{\prime}$ & \\
\hline \multirow{2}{*}{ Rhône aval. $\ldots \ldots \ldots \ldots \ldots \ldots$} & Première onde au-delà de $4 \mathrm{mn} .$. & $-0,5 h^{\prime}$ & \multirow{2}{*}{$-0,67 k^{\prime}$} \\
\hline & Deuxième onde au-delà de $5 \mathrm{mn} .$. & $-0,6 h^{\prime}$ & \\
\hline
\end{tabular}

g) Ondes en aval du bloc usinc-déchargeurécluse :

L'étude des ondes en aval du bloc usinedéchargeur-ćcluse a été menée de manière semblable à ce qui a été exposé pour l'amont. Compte tenu des faibles tirants d'eau, des formes convergentes du garage aval et de l'important retard de transmission au branchement, les ondes qui évoluent entre l'usine et l'écluse (elles ont une période $\mathrm{T}_{2}=2 \mathrm{mn}$ ) présentent une amplitude plus faible et un amortissement beaucoup plus rapide. Les perturbations du plan d'cau en aval de l'usine sont done en général beaucoup moins gênantes que celles de l'amont (voir tabl. VIII).

\section{h) Conclusion :}

L'analyse détaillée des ondes de translation dans les dérivations s'est révélée indispensable pour la mise au point complète des projets. Elle a sans doute permis d'expliquer l'amplitude imprévue de certaines ondes provoquées par des manouvres de l'usine qui pouvaient paraître anodines. Mais elle a surtout permis de choisir à bon escient les caractéristiques utiles du déchargeur et de définir ses conditions d'utilisation (19). En outre, on a pu déterminer, pour chaque tronçon de digues, les revanches nécessaires par rapport aux cotes variables du plan d'eau, et fixer les niveaux maximaux du plan d'eau devant l'usine que l'exploitation ne doit dépasser en aucun cas.

(19) L'ouverture très rapide du déchargeur ne présente d'utilité pour la réduction des ondes que si elle intervient sans retard (quelques secondes) après la disjonction. Passé le délaí d'une demi-minute, une intervention rapide peut devenir nuisible, ear elle risque, par effet de résonance, d'augmenter l'amplitude des ondes, alors que l'ouverture lente (quelques minutes) ne présente pas le même inconvénient. 


\section{4. - CONSIDÉRATIONS FINALES}

L'équipement d'un fleuve comme le Rhône pose toutes sortes de problèmes techniques, parmi lesquels ceux qui relèvent de l'hydraulique jouent évidemment un rôle capital.

Dans les pages qui précèdent, nous avons choisi quelques exemples qui nous ont paru caractéristiques des études effectuées pour les chutes du Bas-Rhône. Certains d'entre eux mettent l'accent sur les problèmes posés par l'instabilité du lit du fleuve et l'importance des débits (liquide ou solide) qui y cheminent. D'autres mettent en relief les problèmes propres aux aménagements mixtes, où la production énergétique, le passage de la navigation fluviale et les intérêts agricoles ou industriels bénéficient d'installations communes. Cette cohabitation nécessaire d'activités diverses, aux objectifs ou aux besoins quelquefois divergents, donne lieu pour chaque chute à des mises au point de nature très variée. Elles sont d'ailleurs d'autant plus délicates qu'il s'agit d'installations de grande taille, qui touchent à des secteurs vitaux de l'économie du pays. Ces études concernent aussi bien le tracé des ouvrages que la définition des modalités d'exécution ou des règles d'exploitation, et font appel à des techniques très diverses.

Presque seule, jusqu'à présent, l'expérimentation systématique sur modèle réduit permettait de compléter les indications très précieuses données par l'intuition, le raisonnement et l'observation des phénomènes naturels; mais il apparait maintenant que l'intervention des calculatrices électroniques peut apporter à son tour un moyen d'investigation très efficace.

Grâce aux exemples choisis, nous avons essayé de dégager les caractères communs aux études faites pour les diverses chutes du bas Rhône. Mais, fort heureusement pour les hydrauliciens, chaque chute qui s'ajoute à la chaîne des aménagements précédents, apporte son lot de problèmes particuliers ou nouveaux, qui soutient l'intérèt de l'équipe d'ingénieurs ou de chercheurs ayant la charge de les résoudre.

BIBLIOGRAPHIE

\section{Barrage el prise}

[1] G. Benini. - Angle de meilleure incidence pour une dérivation, 8 p. Energia Elettrica, juin 1952

[2] G. Benini. - Sur l'angle de prise d'une dérivation. Recherches expérimentales sur le transport des matériaux, 10 p. Energia Elettrica, septembre 1955

[3] J. Chabent, - Résultats principaux de l'étude sur modèle réduit des ouvrages de prise et de retenue de la chute de Montélimar, 23 p. La Houille Blanche, $\mathrm{n}^{\circ} 3$, juillet 1955 .

[4] J. Chaberit. - Etude sur modèle du barrage de retenue de Rochemaure sur le Rhône, 8 p. Construction, t. XII, $\mathrm{n}^{\circ} 4$, avril 1957 .

[5] E. Crausse. - Sur un phénomène d'oscillation du plan d'eau provoqué par l'écoulement autour d'obstacles en forme de piles de pont, 3 p. C.R. Académie des Sciences, t. 209, 24 juillet 1939 (voir p. 197).

Phénomènes oscillatoires dus aux tourbillons alternés, 5 p. Revue Générale de l'Hydraulique, $14^{\circ}$ an., $\mathrm{n}^{\circ} 47$, septembre-octobre 1948.

[6] P. Desattre. - Répartition du débit solide d'un cours d'eau se divisant entre plusieurs bras uaturels ou artificiels, 30 p. $18^{\mathrm{c}}$ Congrès International Navigation, Rome, Ministère de l'Agriculture, 1953.

[7] L. Escande. - Hydraulique générale, 3 vol. $E d$. Privat, Toulouse, 1943 (voir t. III, pp. 249-250).

[8] M. Henry. - L'aménagement mixte de Montélimar sur le secteur médian du Bas-Rhône, 54 p. Travaux, août à novembre 1955 .
[9] G. Lombardi et R. Jupillat. - Etude sur modèle du barrage de retenue de Donzère, $10 \mathrm{p}$. DonzèreMondragon (La Houllle Blanche), mars 1955 (voir p. 55).

[10] G. Lombardi et R. Jupillat. - Etude sur modèle de Ia prise d'eau sur le Rhône, 12 p. DonzèreMondragon (La Houllle Blanche), mars 1955 (voir p. 69).

[11] G. DE Marchi. - Nouvelles recherches expérimentales sur le jaugeur à ressaut hydraulique, $20 \mathrm{p}$. Annales de la Direction des Eaux et dn Génie Rural, 1936 (voir p. 164).

[12] R. Mulder. - Prises d'eau dans les rivières á charriage, 37 p. Wasser und Energie Wirtschaft, septembre-novembre 1955 .

[13] L.J. Trson. - Au sujet de certaines disposilions permettant de réduire les quantités de matière solide entrainée par un système à surface libre, in Transport hydraulique et décantation de matériaux solides, $8 \mathrm{p}$. II tos tournées de l'Hydraulique (La Honille Blanche), juin 1952.

[14] M. Tounasse. - Le problème des entrées à DonzèreMondragon, 4 p. Donzère-Mondragon (La Houllle Blanche), mars 1955 (voir p. 65).

\section{Retenue}

[21] C. Genaehriva. - La chute de Baix-Le Logis Neuf sur le Rhône, 30 p. Travaux, mai 1961. 
222 C. Gemaehring, R. Gnocchio et J. Chabert. - Comparaison entre les évaluations théoriques et expérimentales du débit solide dans le tiers central du Rhône, 14 p. A.t.R.H., $7^{*}$ Congres, Lisbonne, D 21, juillet 1957 .

[23] C. Gexaening et J. Ghabent. - Transport solide et modiffeation du lit d'une riviere a forte pente : la Drôme, 12 p. A.I.H.S., Colloque Bari, octobre 1962.

24] M. Hexny. - Les problèmes liés aux sables et graviers (aménagcment de Donxère-Mondragon), $16 \mathrm{p}$. Donzère-Mondragon (La Houlle Blanche), mars 1955 (voil p. 3!).

[25] M. Henny et C. Gemseruing - - Les mouvements des matériaux dans le lit du Rhòne à la suite de la mise en eau de la dérivation de Donzère-Mondragon, 24 p. La Ilouille Blanche, février 1958.

\section{Usine}

Déchargeur ef ondes de translation

Ecluse et problèmes de navigation

[31] H. Favre. - Etude théorique et expérimentale des ondes de translation dans les canaux decouverts, 215 p. Dinod, 1935.
[32] P. DEAatThe. - Les ouvages de navigation de la chute de Donzère-Mondragon. L'écluse de SaintPierre, 12 p. Travana, $n^{\circ} 211$, février 1952.

[33] C. Genaemling. - Les progrès de l'aménagement du Rhône et de sa voie navigable, 55 p. Bulletin $n^{\circ} 52$ A.I.P.C.N. (Bruxelles), 1960.

34. C. GemaderiNa. - Bibliographie sommaire des principaux ourrages ef articles récents consacrés à l'aménagement du Rhône, $40 \mathrm{p}$. Bulletin $n^{\circ} 52$ de A.X.P.C.N. (Bruxelles), 1960.

[35] M. HeNry. - L'aménagement du tiers central du bas-Rhône, 14 p. Travaux, mai 1956.

[36] M. Henry. - L'aménagement de la voie navigable du bas-Rhône, 17 p. Travanx, septembre 1961 .

[37 E. Pariskx. - Caractéristiques du fonctionmement hydranlique de l'écluse de Bollène, $10 \mathrm{p}$. DonzèreMondragon (La Houlle Blanche), mars 1955 (voir p. 137$)$.

[38] E. Pariset et M. Grtiwith-Nemoz. - Etude hydraulique de l'aménagement de Donzère-Mondragon aux abords de la centrale André Blondel, 24 p. Donzère-Mondragon (La Honille Blanche), mars 1955 (voir p. 101).

[39] M. Tovrasse. - Le problème des coupures et du rétablissement rapide du débit, 4 p. Donzère. Mondragon (La Honille Blanche), mars 1955 (voir p. 125).

\section{DISCUSSION}

Président: M. Chapovthish

M. le Président remercie M. GaYET (1) et sonligne trois choses:

1. L'expression d"oménagement est revenue très souvent, signifiant que la notion d'usine est dépassée. Même lorsqu'il y at une série assez homogène d'usines qui se ressemblent beaucoup, c'est l'ensemble de toutes ces usines qui finit par constituer un complexe hydraulique qu'il faut regarder dans son tout.

$2^{*}$ Il semble qu'on n'avait jamais pratiqué d'une manière aussi systematique cette association entre le modele reduit et la calculatrice électronique.

3" On constate ici que, d'une manière assez insolite, une usine peut être en amont de son barrage, ce qui est le cas de pierre-Bénite.

M. Bonnemox demande s'll a été établi une corrélation entre la granulométrie du debit solide par un seuil d'entrée et la dénivellation de ee scuil par rapport au lit de la rivière.

H. GaYet ne pense pas qüil existe une relation bien définic entre la grannlométric des matériaux arctés et la dénivellation du seuil d'entrée. En effet, comme cela a été exposé, la tendance plus ou moins grande des materiaux à pénétrer dans la prise dépend d'autres parametres (notamment de la courbure du lit); si une telle relation pouvait être mise en ividence, elle varierait probablement d'un aménagement à l'autre.

Il convient dailleurs de distinguer, dans le cas du Rhone tout au moins :

- les materiaux fins, qui sont transportés en suspension; ces matóriaux pénètrent en majeure partie daus le canal et transitent rers l'aval; une faible quantité peut se cléposer dans cerlaines zones de l'aménagement (garages de navigation, par excmple); mais la dénivellation du seuil est pratiquement sans influence sur la pénétration de ces materiaux dans le canal;

(1) Catte communication a bte exposie par M. Gayet, Ingénew a la C.N.R. sapplent M. Gemamisat, qui a presenté ses excuses de ne pouvoir exposer que les deux premieres parties. La trobsieme partie sera presentè à une prochaine session.
- les matériaux grossiers, qui se déplacent sur le fond du lit; avec les formes d'entrée étudíes, ces matériaux transitent normalement vers le barragc; il semble que les dénivellations des seuils pourraient itre un peu moins importantes que celles adoptées, sans pour cela que les matériaux grossiers aient tendance à pénétrer dans le canal.

M. CHabert ajoute que, pour la plupart de ces aménagements, le seuil d'entréc de la prise d'eau est situé $4 \mathrm{~m}$ an-dessus de la cote du radier du barrage.

Les essais systématiques effectués sur modèle ont montré que cette marge étalt suflisante, compte tenu des formes d'entrées mises au point, pour éviter l'engravement de Ja prise; la cote du seuil dépend moins de la granulométrie des alluvions que de la hauteur de l'engravement, qui peut se produire à longue échéance suivant les conditions hydrauliques réalisées par le barrage (retenue ou chasse).

M. Lefouton indique qu'il a adopté cette marge de $4 \mathrm{~m}$ pour les aménagements du Rhin, à titre, surtout, de protection pendant les périodes de erue: les remous ntteignant des Iongueurs de $8 \mathrm{~km}$ pendant ces périodes, une partie des matériaux charriés en exploitation normale est déposéc bien en amont et protège le canal d'amenée. Les vitesses dans les canaux d'amenée étant comprises entre $1,20 \mathrm{~m}$ et $0,40 \mathrm{~m}$ par seconde, l'on admet que les matériaux fins sont toujours entraînés.

Par aillears, M. Lmovron explique qu'il a eu un problème délicat à résoudre; l'élimination des boues salées très fines des Potasses d'Alsace qui se déversent dans le Rhin et représentent un débit solide important. De manière à he pas engraver le canal d'amenée, ces boues ont été rejetés immédiatement à l'aval d'une usine, dans la zone de turbujence des aspirateurs des turbines et des déchargeurs, par quatre condrites d'évacuation associées aux quatre turbines. Des vannes permettent de dispatcher l'effluent vers les turbines en fonctionnement. Une itude sur modèle a permis de s'assurer que les boues ne se déposaient pas sur la distance de $15 \mathrm{~km}$ qui sćpare deux usines et qu'elles étaient remises en suspension par la turbulence des turbines de l'usine suivante. 